TRANSACTIONS OF THE

AMERICAN MATHEMATICAL SOCIETY

Volume 355, Number 9 , Pages 3537-3590

S 0002-9947(03)03137-4

Article electronically published on May 29, 2003

\title{
A FREE BOUNDARY PROBLEM FOR A SINGULAR SYSTEM OF DIFFERENTIAL EQUATIONS: AN APPLICATION TO A MODEL OF TUMOR GROWTH
}

\author{
SHANGBIN CUI AND AVNER FRIEDMAN
}

\begin{abstract}
In this paper we consider a free boundary problem for a nonlinear system of two ordinary differential equations, one of which is singular at some points, including the initial point $r=0$. Because of the singularity at $r=0$, the initial value problem has a one-parameter family of solutions. We prove that there exists a unique solution to the free boundary problem. The proof of existence employs two "shooting" parameters. Analysis of the profiles of solutions of the initial value problem and tools such as comparison theorems and weak limits of solutions play an important role in the proof. The system considered here is motivated by a model in tumor growth, but the methods developed should be applicable to more general systems.
\end{abstract}

\section{INTRODUCTION}

A special feature in tumor growth is proliferation; proliferating cells cause the tumor volume to vary in time, and, as a result, various models developed to describe tumor growth are formulated as initial boundary value problems for partial differential equations with the tumor surface as a free boundary.

It has long been recognized that a tumor contains different populations of cells, such as proliferating cells (i.e., cells that undergo abnormally fast mitosis), necrotic cells (i.e., cells that died because of lack of nutrition) and "in-between" quiescent cells (i.e., cells that are alive but their rate of mitosis is balanced by the rate of natural death).

There are basically two kinds of models in the literature, according to which one of the following two assumptions is adopted:

(i) different populations are segregated by interface boundaries;

(ii) different populations are mixed together in different concentrations.

Models with segregated populations were developed by Greenspan [9], 10], Adam [1], Britton and Chaplain [2] and Byrne and Chaplain [3] (see also the references therein). In such models, necrotic cells occupy a central core $r<\rho_{1}(t)$, proliferating cells occupy an outer layer $\rho_{2}(t)<r<R(t)$, and quiescent cells reside in the shell $\rho_{1}(t)<r<\rho_{2}(t)$; in these regions nutrient and inhibitor concentrations satisfy reaction-diffusion equations. Rigorous analysis of models of this kind was given by

Received by the editors March 15, 2002.

2000 Mathematics Subject Classification. Primary 34B15; Secondary 35C10, 35Q80, 92C15.

Key words and phrases. Free boundary problem, stationary solutions, singular differential equations, tumor growth. 
Friedman and Reitich 7] and Cui and Friedman [4] (for the case $\rho_{1}(t)=\rho_{2}(t)=0$ ) and by Cui and Friedman [5] (for the case $\rho_{1}(t)=\rho_{2}(t)>0$ ).

Models of the type (ii) were more recently developed in Ward and King [16], Pettet et al. [13] and Sherrat and Chaplain [14]. In this paper we are particularly interested in the model of Pettet et al. 13]. This model was developed in order to explain the experimental results of Dorie et al. 6], 7] with regard to internalization of particles injected into the tumor across its surface. Previous models introduced in order to explain the same experimental results were given by McElwain and Pettet [12] and Thompson and Byrne [15].

As in other tumor models, the one developed in 13. considers all nutrients as a single species, and assumes that its concentration $C$ satisfies the diffusion equation

$$
\nabla^{2} C=\mu C,
$$

where $\mu$ is a positive constant, accounting for the consumption rate of nutrient divided by the diffusion coefficient.

Another basic assumption adopted by [13] is that dead cells are withdrawn immediately from the tumor upon their death, so that the tumor contains only living cells. It is also assumed that all cells are incompressible and of the same volume, and that the tumor is a spheroid well packed with cells. It follows that the cell density within the tumor is a constant, say, $N$. Thus, denoting by $P$ and $Q$ the densities of the proliferating cells and quiescent cells, respectively (i.e., the numbers of proliferationg cells and quiescent cells per unit volume, respectively), we get

$$
P+Q=N \text {. }
$$

Next, proliferating cells are assumed to undergo mitosis at a rate $K_{B}(C)$ and become quiescent at a rate $K_{Q}(C)$; the quiescent cells are assumed to revert to proliferating cells at a rate $K_{P}(C)$ and undergo necrosis at a rate $K_{D}(C)$. This implies, by the law of conservation of mass, that

$$
\begin{aligned}
& \frac{\partial P}{\partial t}+\nabla \cdot\left(\vec{u}_{P} P\right)=\left(K_{B}(C)-K_{Q}(C)\right) P+K_{P}(C) Q, \\
& \frac{\partial Q}{\partial t}+\nabla \cdot\left(\vec{u}_{Q} Q\right)=K_{Q}(C) P-\left(K_{D}(C)+K_{P}(C)\right) Q,
\end{aligned}
$$

where $\vec{u}_{P}$ and $\vec{u}_{Q}$ are the velocities of proliferating cells and quiescent cells, respectively. The functions $K_{B}(C), K_{D}(C), K_{P}(C)$ and $K_{Q}(C)$ are taken to be linear functions:

$$
\begin{array}{ll}
K_{B}(C)=k_{B} C, & K_{D}(C)=k_{D}\left(C_{0}-C\right), \\
K_{P}(C)=k_{P} C, & K_{Q}(C)=k_{Q}\left(C_{0}-C\right),
\end{array}
$$

where $C_{0}$ is a positive constant, and the coefficients $k_{B}, k_{D}, k_{P}, k_{Q}$ are typically given by

$$
k_{P}=0.05, \quad k_{D}=0.1, \quad k_{B}=1, \quad k_{Q}=0.9 .
$$

The velocities $\vec{u}_{P}$ and $\vec{u}_{Q}$ are mutually related by the equation

$$
\vec{u}_{Q}=\vec{u}_{P}+\chi \nabla C,
$$

where $\chi$ is a chemotactic sensitivity coefficient, assumed to be a nonnegative constant. The last assumption is based on some evidence that proliferating cells seem to be less motile as they undergo mitosis [11]. 
Introducing the mean velocity

$$
\vec{u}=\frac{1}{N}\left(P \vec{u}_{P}+Q \vec{u}_{Q}\right)
$$

we get from (1.3) and (1.4) the equation

$$
\nabla \cdot \vec{u}=\frac{1}{N}\left\{K_{B}(C) P-K_{D}(C) Q\right\} .
$$

Finally, the movement of the tumor surface is assumed to be governed by the equation of continuity, namely,

$$
\frac{d R}{d t}=\vec{u} \cdot \vec{n},
$$

where $R=R(t)$ is the radius of the tumor spheroid and $\vec{n}$ represents the outward normal to the tumor surface.

Equations (1.1)-(1.9) complemented by appropriate boundary and initial conditions form a model of evolution of a tumor containing only cells that are alive but in two different states (proliferating and quiescent) co-inhabiting the tumor. We are interested in developing a rigorous mathematical treatment of this model. The present paper considers stationary solutions for the case $\chi=0$; non-stationary solutions and the more difficult case $\chi \neq 0$ will be studied in future work.

After rescaling space and time, and setting

$$
p=\frac{P}{N}, \quad q=\frac{Q}{N}=1-p, \quad c=\frac{C}{C_{0}}, \quad \vec{u}=u \frac{x}{|x|},
$$

the stationary problem can be reformulated in the following form:

$$
\begin{gathered}
c^{\prime \prime}+\frac{2}{r} c^{\prime}=\mu c, \quad 0<r<R, \\
c^{\prime}(0)=0, \quad c(R)=1, \\
u^{\prime}+\frac{2}{r} u=-K_{D}(c)+K_{M}(c) p, \quad 0<r<R, \\
u(0)=0, \\
u p^{\prime}=K_{P}(c)+\left(K_{M}(c)-K_{N}(c)\right) p-K_{M}(c) p^{2}, \quad 0<r<R, \\
u(R)=0,
\end{gathered}
$$

where

$$
K_{M}=K_{B}+K_{D}, \quad K_{N}=K_{P}+K_{Q},
$$

and $K_{B}(c), K_{D}(c), K_{P}(c)$ and $K_{Q}(c)$ are rescaled forms of the corresponding functions given by (1.5), that is,

$$
\begin{aligned}
& K_{B}(c)=C_{0} k_{B} c, K_{D}(c)=C_{0} k_{D}(1-c), \\
& K_{P}(c)=C_{0} k_{P} c, \quad K_{Q}(c)=C_{0} k_{Q}(1-c) .
\end{aligned}
$$

Indeed, equation (1.10) is the radially symmetric form of (1.1). The first boundary condition in (1.11) follows from the radial symmetry of the solution of the differential equation for $c$, and the second boundary condition means that the tumor receives sufficient nourishment from its host tissue (recall that $K_{Q}(1)=0$ and $K_{D}(1)=0$, which means that proliferating cells do not become quiescent and quiescent cells do not undergo necrosis when the level of nourishment is $c=1$ ). Equation 
(1.12) is the radially symmetric version of (1.8) (with $q$ replaced by $1-p$ ). If we substitute (1.12) into (1.3) and use the assumption that $p$ is independent of $t$ and $\chi=0$, we obtain the equation (1.14). The boundary condition (1.13) is a consequence of radial symmetry of $\vec{u}$. Finally, the free boundary condition (1.15) is the stationary form of (1.9).

The main result of this paper is the following: The stationary problem (1.10)(1.16) has a unique solution $(c, p, u, R)$ with the properties

$$
0<c(r)<1, \quad c^{\prime}(r)>0, \quad 0<p(r)<1, \quad p^{\prime}(r)>0, \quad u(r)<0 \quad \text { for } 0<r<R .
$$

The last inequality means that cells are moving into the interior of the tumor. Actually, our analysis does not depend on the linearity of the functions in (1.17), nor does it depend on the special form of the right-hand side of (1.10). In $\S 2$ we shall state our main result in a more general form and then outline the structure of this paper.

We conclude this section by calling attention to the paper of Ward and King 16. This paper introduces a model for a spheroid tumor with two populations of cells: proliferating cells and dead cells. The model is similar to that of Pettet et al. [13] with $\chi=0$. However, the assumptions made on the coefficients which appear in the equations that are analogous to (1.3), (1.4) are quite different; thus, for example, the function $K_{B}+K_{D}$ in [13] is uniformly positive whereas in [16] it changes sign. In [16] the radius $R(t)$ of the tumor is shown, numerically, to increase to infinity at a linear rate, so that one cannot expect to have stationary solutions.

\section{The MAIn RESULT}

Consider the following free boundary problem:

$$
\begin{gathered}
u(r) p^{\prime}(r)=K_{P}(c(r))+\left(K_{M}(c(r))-K_{N}(c(r))\right) p(r)-K_{M}(c(r)) p^{2}(r), 0<r<R, \\
u(R)=0,
\end{gathered}
$$

with solution $(c, u, p, R)$ satisfying

$$
c \in C[0, R] \cap C^{2}(0, R), \quad u \in C[0, R] \cap C^{1}(0, R), \quad p \in C[0, R] \cap C^{1}(0, R)
$$

and

$$
c(r) \geq 0, \quad 0 \leq p(r) \leq 1 \text { for } 0 \leq r \leq R .
$$

The functions on the right-hand side of (2.1), (2.3) and (2.5) satisfy the following assumptions:

$$
\begin{gathered}
F(c), K_{D}(c), K_{M}(c), K_{N}(c) \text { and } K_{P}(c) \text { are analytic in } c, 0 \leq c \leq 1 \\
F(0)=0, \quad F^{\prime}(c)>0 \text { for } 0 \leq c \leq 1
\end{gathered}
$$




$$
\left\{\begin{array}{c}
K_{D}^{\prime}(c)<0 \text { for } 0 \leq c \leq 1, \text { and } K_{D}(1)=0 \\
K_{P}^{\prime}(c)>0 \text { for } 0 \leq c \leq 1, \text { and } K_{P}(0)=0 \\
K_{M}(c)=K_{B}(c)+K_{D}(c), \text { where } K_{B}(c) \text { satisfies the same } \\
\text { conditions as } K_{P}(c) \text { and } K_{B}^{\prime}(c)+K_{D}^{\prime}(c)>0 \text { for } 0 \leq c \leq 1 \\
K_{N}(c)=K_{P}(c)+K_{Q}(c), \text { where } K_{Q}(c) \text { satisfies the same } \\
\text { conditions as } K_{D}(c)
\end{array}\right.
$$

The conditions on $K_{B}(c)$ and $K_{D}(c)$ imply that

$$
K_{M}(c)>0, \quad K_{M}^{\prime}(c)>0 \text { for } 0 \leq c \leq 1
$$

Note that the conditions in (2.11) are clearly satisfied for the functions in (1.17); in particular, the inequality $K_{B}^{\prime}(c)+K_{D}^{\prime}(c)>0$ follows from the assumption $k_{B}>k_{D}$.

The main result of this paper is the following:

Theorem 2.1. Under the assumptions (2.9)-(2.11), the free boundary problem (2.1)-(2.6) has a unique solution $(c, p, u, R)$ with $R>0, c$ analytic in $[0, R], p$ and $u$ continuous in $[0, R]$ and analytic in $(0, R]$, and

$$
\begin{gathered}
0<c(r)<1, \quad c^{\prime}(r)>0 \quad \text { if } 0<r<R, \quad c(0)>0, \\
0<p(r)<1, \quad p^{\prime}(r)>0 \quad \text { if } 0<r<R, \quad p(0)>0, \\
p(R)=1, \quad p^{\prime}(R)>0, \\
u(r)<0 \quad \text { if } 0<r<R .
\end{gathered}
$$

We shall use the shooting method to prove the existence result. The main idea is as follows:

For each $\lambda \in(0,1)$ we denote by $c_{\lambda}(r)$ the solution of (2.1) with initial value

$$
c_{\lambda}(0)=\lambda, \quad c_{\lambda}^{\prime}(0)=0 .
$$

One can easily verify that $c_{\lambda}(r)$ is analytic for $r \geq 0, c_{\lambda}^{\prime}(r)>0, \frac{\partial c_{\lambda}(r)}{\partial \lambda}>0$ for $r>0, c_{\lambda}^{\prime \prime}(0)>0$, and there exists a unique finite number $R_{\lambda}>0$ such that

$$
c_{\lambda}\left(R_{\lambda}\right)=1
$$

Furthermore, $\frac{d}{d \lambda} R_{\lambda}<0$ for $0<\lambda<1, R_{\lambda} \rightarrow \infty$ if $\lambda \rightarrow 0$, and $R_{\lambda} \rightarrow 0$ if $\lambda \rightarrow 1$. We substitute $c(r)=c_{\lambda}(r)$ into the system $(2.3)-(2.5)$ to get

$$
\begin{gathered}
u(r) p^{\prime}(r)=K_{P}\left(c_{\lambda}(r)\right)+\left(K_{M}\left(c_{\lambda}(r)\right)-K_{N}\left(c_{\lambda}(r)\right)\right) p(r)-K_{M}\left(c_{\lambda}(r)\right) p^{2}(r), \\
0<r<R_{\lambda} .
\end{gathered}
$$

Hence the problem of solving the system (2.1)-(2.6) is transformed into the following problem: Find values of $\lambda$ and corresponding solutions of (2.19)-(2.21) such that the free boundary condition

$$
u\left(R_{\lambda}\right)=0
$$

is satisfied. 
Due to the singularity at $r=0$ of the differential equations (2.19), (2.21), the shooting method we use in this paper turns out to be quite different from the standard one. Indeed, we shall prove that, unlike other nonsingular ODE problems, solutions of the initial value problem (IVP) (2.19)-(2.21) exihibit the following interesting phenomenon: There exists a critical value $\lambda_{\infty} \in(0,1)$ such that, for any $\lambda_{\infty}<\lambda<1$, the IVP has a unique solution, while for each $0<\lambda<\lambda_{\infty}$, the IVP has a continuum of solutions $\left(p_{\lambda \psi}, u_{\lambda \psi}\right)(\psi \in \mathbf{R})$. We shall also prove that the value of $\lambda$ for which the free boundary problem (FBP) (2.19)-(2.22) has a solution must belong to $\left(0, \lambda_{\infty}\right)$. Thus, our "shooting target" has to be reached in two steps: First, for every $\lambda \in\left(0, \lambda_{\infty}\right)$ we need to find a $\bar{\psi} \in \mathbf{R}$ such that the solution $\left(p_{\lambda}, u_{\lambda}\right) \equiv\left(p_{\lambda \bar{\psi}}, u_{\lambda \bar{\psi}}\right)$ of the IVP possesses the "best" approximate properties to the solution of the FBP. Having determined this special solution $\left(p_{\lambda}, u_{\lambda}\right)$ for each $\lambda \in\left(0, \lambda_{\infty}\right)$, we then proceed with the second step of determining a value of $\lambda$ for which $\left(p_{\lambda}, u_{\lambda}\right)$ satisfies the free boundary condition (2.22).

The structure of the paper is as follows:

In $\S 3$ we introduce some auxiliary functions of $\lambda$ and study their basic properties. These functions play a fundamental role throughout this paper.

In order to prove Theorem 2.1 we first need to solve the IVP near $r=0$. Since the equations (2.19) and (2.21) (particularly the second one) are singular at $r=0$, local existence of solutions of the IVP is not ensured by classical results. Sectons 4 and 5 are devoted to establishing local existence (i.e., for $0<r<\delta, \delta$ small) of solutions for the IVP. In $\S 4$ we consider only analytic solutions. We prove that there exists a sequence $\left\{\lambda_{n}\right\}_{n=1}^{\infty}$ converging to $\lambda_{\infty}$ increasingly, such that for each $\lambda \neq \lambda_{n},(2.19)-(2.21)$ has a unique analytic solution, while for each $\lambda_{n}$, the system has either no analytic solutions or a continuum of analytic solutions.

In $\S 5$ we consider the IVP for non-analytic solutions. The key step is to transform the IVP into an equivalent system of integral equations that can be solved by using the contraction mapping principle. The final result is as follows: for each $\lambda_{\infty}<\lambda<1$, there is a unique solution, whereas for each $0<\lambda<\lambda_{\infty}$, there exists a continuum of classical solutions $(p(r ; \lambda, \omega), u(r ; \lambda, \omega))$ depending on a real parameter $\omega$.

The family of solutions $(p(r ; \lambda, \omega), u(r ; \lambda, \omega))$ does not depend continuously on $(\lambda, \omega)$ at the points $\lambda=\lambda_{n}$. To overcome this difficulty, we introduce, in $\S 6$, a parameterization $\omega=\omega(\lambda, \psi)$ with a new parameter $\psi \in \mathbf{R}$ such that the solutions will depend continuously on $(\lambda, \psi)$ for all $\lambda \in\left(0, \lambda_{\infty}\right)$ and $\psi \in \mathbf{R}$ (for $0 \leq r<\delta$, for some $\delta>0)$. This solution will be denoted by $\left(p_{\lambda \psi}, u_{\lambda \psi}\right)$.

The next step is to study the profiles of $p_{\lambda \psi}(r)$ and $u_{\lambda \psi}(r)$ and use this information to extend the solution to either the entire interval $\left[0, R_{\lambda}\right]$ or a maximal interval $[0, \bar{R})$ such that $p_{\lambda \psi}(r)$ blows up at $r=\bar{R}$. This is done in $\S 7$, where we shall prove, in particular, that $p_{\lambda \psi}(r)$ can change monotonicity (from increasing to decreasing) at most once. It will also be clear from the discussion of this section that the values of $\lambda$ for which the free boundary condition (2.22) can be satisfied must belong to $\left(0, \lambda_{\infty}\right)$.

In $\S 8$ we derive another integral equation formulation of the IVP and use it to introduce the concept of weak solutions. Unlike the integral equation formulation introduced in $\S 5$, which holds only locally, the integral equation formulation derived in this section holds globally. It will enable us to work with weak limits of solutions. The main result of this section asserts that a weak solution $(p, u)$ of the IVP is actually a classical solution provided $p \geq 0$. 
In $\S 9$ we shall perform the first step of the shooting method; that is, we shall prove that for any $\lambda \in\left(0, \lambda_{\infty}\right)$ there is a unique $\bar{\psi}$ such that $\left(p_{\lambda}, u_{\lambda}\right) \equiv\left(p_{\lambda \bar{\psi}}, u_{\lambda \bar{\psi}}\right)$ is either a "subsolution" or a "supersolution" of the FBP. By a subsolution of the FBP we mean a solution $(p(r), u(r))$ of the IVP, defined for all $0 \leq r \leq R_{\lambda}$, that satisfies the conditions

$$
\begin{aligned}
0<p(r)<1, & p^{\prime}(r)>0 \text { for } 0<r<R_{\lambda}, \\
u(r)<0 \text { for } 0<r<r_{0}, & u\left(r_{0}\right)=0, \quad u(r)>0 \text { for } r_{0}<r \leq R_{\lambda},
\end{aligned}
$$

for some $0<r_{0} \leq R_{\lambda}$. By a supersolution of the FBP we mean a solution $(p(r), u(r))$, defined also for all $0 \leq r \leq R_{\lambda}$, that satisfies the conditions

$$
0<p(r)<1, \quad p^{\prime}(r)>0, \quad u(r)<0 \text { for } 0<r<R_{\lambda},
$$

and $p\left(R_{\lambda}\right)=1, u\left(R_{\lambda}\right)<0$. The discussion of this section shows that subsolutions and supersolutions possess the "best" approximate properties to the solution of the FBP among all solutions of the IVP. We shall also prove that a unique subsolution exists for each $\lambda$ near $\lambda_{\infty}$.

In $\S 10$ we shall perform the second step of the shooting method to get a solution of the FBP. First, we prove that the three sets

$$
\begin{aligned}
B_{0} \equiv\left\{\lambda \in\left(0, \lambda_{\infty}\right):\right. & \lambda \text { corresponds to a solution of the } \mathrm{FBP}\} \\
B_{1} \equiv\left\{\lambda \in\left(0, \lambda_{\infty}\right):\right. & \lambda \text { corresponds to a subsolution, } \\
& \text { but not a solution of the FBP }\}, \\
B_{2} \equiv\left\{\lambda \in\left(0, \lambda_{\infty}\right):\right. & \lambda \text { corresponds to a supersolution of the FBP }\}
\end{aligned}
$$

do not intersect each other and their union is equal to $\left(0, \lambda_{\infty}\right)$. Next, we prove that $B_{1}$ and $B_{2}$ are open sets. Finally, we show that every $\lambda$ near 0 belongs to $B_{2}$, so that $B_{2} \neq \varnothing$. Since also $B_{1} \neq \varnothing$ (by $\left.\S 9\right)$, we conclude that $B_{1} \cup B_{2} \neq\left(0, \lambda_{\infty}\right)$, so that $B_{0} \neq \varnothing$, which means that there exists at least one solution of the free boundary problem. Uniqueness is proved in $\$ 11$.

Some formulas used in $\S 5$ and $\S 6$ are proved in the Appendix $(\S 12)$.

To end this section we want to emphasize that throughout this paper we consider only solutions that satisfy the condition $p(0) \geq 0$; this is of course motivated by the fact that $p(r)$ represents the density of cells. The condition $p(0) \geq 0$ appears implicitly in several places in this paper.

\section{Auxiliary functions}

In this section we introduce several functions of $\lambda$ that will play a fundamental role throughout this paper.

Lemma 3.1. For any $0<\lambda \leq 1$ the quadratic equation

$$
K_{P}(\lambda)+\left(K_{M}(\lambda)-K_{N}(\lambda)\right) \alpha-K_{M}(\lambda) \alpha^{2}=0
$$

has a unique positive solution, which we denote by $\alpha(\lambda)$, and

$$
\begin{gathered}
0<\alpha(\lambda)<1, \quad \alpha^{\prime}(\lambda)>0 \text { for } 0<\lambda<1, \quad \alpha(1)=1, \\
\alpha(0+)=\left\{\begin{array}{l}
0 \text { if } K_{D}(0) \leq K_{Q}(0), \\
\left(K_{D}(0)-K_{Q}(0)\right) / K_{D}(0) \text { if } K_{D}(0)>K_{Q}(0) .
\end{array}\right.
\end{gathered}
$$


Proof. Denote by $G(\alpha, \lambda)$ the left-hand side of the equation (3.1). It is clear that for all $0<\lambda<1$,

$$
\begin{gathered}
G(1, \lambda)=K_{P}(\lambda)-K_{N}(\lambda)=-K_{Q}(\lambda)<0, \\
G(0, \lambda)=K_{P}(\lambda)>0, \quad \lim _{\alpha \rightarrow \pm \infty} G(\alpha, \lambda)=-\infty .
\end{gathered}
$$

Hence the equation (3.1) has exactly one positive root in the interval $(0,1)$. It is given by

$$
\alpha(\lambda)=\frac{1}{2 K_{M}(\lambda)}\left(K_{M}(\lambda)-K_{N}(\lambda)+\sqrt{\left(K_{M}(\lambda)-K_{N}(\lambda)\right)^{2}+4 K_{M}(\lambda) K_{P}(\lambda)}\right) .
$$

By (2.11) and (2.12), it follows that

$$
\begin{aligned}
\frac{\partial G(\alpha, \lambda)}{\partial \lambda} & =K_{P}^{\prime}(\lambda)+\left(K_{M}^{\prime}(\lambda)-K_{N}^{\prime}(\lambda)\right) \alpha-K_{M}^{\prime}(\lambda) \alpha^{2} \\
& =K_{P}^{\prime}(\lambda)(1-\alpha)-K_{Q}^{\prime}(\lambda) \alpha+K_{M}^{\prime}(\lambda) \alpha(1-\alpha)>0
\end{aligned}
$$

for $0<\alpha<1,0<\lambda<1$, and, by (3.2),

$$
\begin{aligned}
\frac{\partial G}{\partial \alpha}(\alpha(\lambda), \lambda) & =\left(K_{M}(\lambda)-K_{N}(\lambda)\right)-2 K_{M}(\lambda) \alpha(\lambda) \\
& =-\sqrt{\left(K_{M}(\lambda)-K_{N}(\lambda)\right)^{2}+4 K_{M}(\lambda) K_{P}(\lambda)}<0
\end{aligned}
$$

for $0<\lambda<1$, so that

$$
\alpha^{\prime}(\lambda)=-\frac{\partial G}{\partial \lambda}(\alpha(\lambda), \lambda) / \frac{\partial G}{\partial \alpha}(\alpha(\lambda), \lambda)>0
$$

for $0<\lambda<1$. The rest follows immediately from (3.2).

By the above lemma, it is reasonable to define $\alpha(0)=\alpha(0+)$. From (3.2) one easily finds that $\alpha(\lambda)$ is also differentiable at $\lambda=0,1$, and $\alpha^{\prime}(0)>0, \alpha^{\prime}(1)>0$. We introduce the function

$$
\beta(\lambda)=\frac{1}{3}\left(-K_{D}(\lambda)+K_{M}(\lambda) \alpha(\lambda)\right), \quad 0 \leq \lambda \leq 1 .
$$

Lemma 3.2. We have $\beta^{\prime}(\lambda)>0$ for all $0 \leq \lambda \leq 1$, and there exists a unique number $\lambda_{\infty} \in(0,1)$ such that

$$
\beta(\lambda)\left\{\begin{array}{l}
<0 \text { for } 0 \leq \lambda<\lambda_{\infty}, \\
=0 \text { for } \lambda=\lambda_{\infty} \\
>0 \text { for } \lambda_{\infty}<\lambda \leq 1 .
\end{array}\right.
$$

Proof. Since $K_{M}(\lambda)=K_{B}(\lambda)+K_{D}(\lambda)$ and $K_{B}^{\prime}(\lambda)>0, K_{D}^{\prime}(\lambda)<0$, we have

$$
\beta^{\prime}(\lambda)=\frac{1}{3}\left(-K_{D}^{\prime}(\lambda)(1-\alpha(\lambda))+K_{B}^{\prime}(\lambda) \alpha(\lambda)+K_{M}(\lambda) \alpha^{\prime}(\lambda)\right)>0
$$

for $0 \leq \lambda \leq 1$. Next we note that

$$
\beta(0)=-\frac{1}{3} K_{D}(0)(1-\alpha(0))<0 \quad \text { and } \beta(1)=\frac{1}{3} K_{B}(1)>0 .
$$

Hence there exists a unique $\lambda=\lambda_{\infty}$ such that (3.7) holds.

For every integer $n \geq 0$ we introduce the function

$$
\begin{aligned}
\gamma_{n}(\lambda) & =n \beta(\lambda)-\left(K_{M}(\lambda)-K_{N}(\lambda)\right)+2 K_{M}(\lambda) \alpha(\lambda) \\
& =n \beta(\lambda)+\sqrt{\left(K_{M}(\lambda)-K_{N}(\lambda)\right)^{2}+4 K_{M}(\lambda) K_{P}(\lambda)},
\end{aligned}
$$

where $0 \leq \lambda \leq 1$. 
Lemma 3.3. (1) If $\lambda_{\infty} \leq \lambda \leq 1$, then $\gamma_{n}(\lambda)>0$ for all $n$, and if $0 \leq \lambda<\lambda_{\infty}$, then

$$
\begin{gathered}
\gamma_{1}(\lambda)>\gamma_{2}(\lambda)>\cdots>\gamma_{n}(\lambda)>\gamma_{n+1}(\lambda)>\cdots \\
\lim _{n \rightarrow \infty} \gamma_{n}(\lambda)=-\infty .
\end{gathered}
$$

(2) There exists a positive integer $n_{0}$ such that for every $n \geq n_{0}$ the following assertions hold:

(a) $\gamma_{n}^{\prime}(\lambda)>0$ for $0 \leq \lambda \leq 1$,

(b) the equation $\gamma_{n}(\lambda)=0$ has a unique positive root $\lambda_{n}$, and

(c) the sequence $\left\{\lambda_{n}\right\}_{n=n_{0}}^{\infty}$ is monotone increasing and

$$
\lim _{n \rightarrow \infty} \lambda_{n}=\lambda_{\infty}
$$

(3) For $1 \leq n<n_{0}$, the set of zeros of $\gamma_{n}(\lambda)$ is either finite or empty.

Proof. Assertion (1) follows immediately from Lemma 3.2. Next we note that $\gamma_{n}^{\prime}(\lambda)$ is the sum of $n \beta^{\prime}(\lambda)$ and a bounded continuous function. Since

$$
\beta^{\prime}(\lambda) \geq \text { const. }>0
$$

for $0 \leq \lambda \leq 1$, it follows that (a) holds for sufficiently large $n$. Thus, for large $n$, $\gamma_{n}(\lambda)$ cannot have more than one zero. Since

$$
\gamma_{n}\left(\lambda_{\infty}\right)=\sqrt{\left(K_{M}\left(\lambda_{\infty}\right)-K_{N}\left(\lambda_{\infty}\right)\right)^{2}+4 K_{M}\left(\lambda_{\infty}\right) K_{P}\left(\lambda_{\infty}\right)}>0, \quad n=1,2, \cdots,
$$

and, for large $n$,

$$
\gamma_{n}(0)=-\frac{n}{3} K_{D}(0)(1-\alpha(0))+\left|K_{D}(0)-K_{Q}(0)\right|<0,
$$

we see that (b) holds also for sufficiently large $n$. The assertion that $\left\{\lambda_{n}\right\}_{n=n_{0}}^{\infty}$ is monotone increasing follows from (3.9) and the fact that $\gamma_{n}^{\prime}(\lambda)>0$ for large $n$. To prove (3.10) we note, by (3.8), that

$$
\beta\left(\lambda_{n}\right)=-\frac{1}{n} \sqrt{\left(K_{M}\left(\lambda_{n}\right)-K_{N}\left(\lambda_{n}\right)\right)^{2}+4 K_{M}\left(\lambda_{n}\right) K_{P}\left(\lambda_{n}\right)} \rightarrow 0
$$

as $n \rightarrow \infty$. Since $\beta\left(\lambda_{\infty}\right)=0$ and $\beta^{\prime}(\lambda) \geq$ const. $>0$ for $0 \leq \lambda \leq 1$, the assertion (3.10) follows. Finally, the assertion (3) follows from the fact that $\gamma_{n}(\lambda)$ is analytic in $\lambda$ for all $0 \leq \lambda \leq 1$.

\section{Analytic solutions of the initial value problem}

In order to solve the free boundary problem (2.19)-(2.22), we first need to investigate general solutions of the initial value problem (2.19)-(2.21). Since, by (2.20), the equation (2.21) is singular at $r=0$, the existence of solutions of this problem does not follow from a standard theory. To get an insight into the construction of the general solution, we begin by considering solutions that are analytic near $r=0$.

The main result of this section is the following:

\section{Theorem 4.1. Let}

$$
S=\left\{\lambda \in(0,1): \gamma_{n}(\lambda)=0 \text { for some integer } n \geq 1\right\} .
$$

Then, for any $\lambda \in(0,1) \backslash S$, there exists a unique analytic solution of $(2.19)-(2.21)$ in some interval $0 \leq r \leq \delta, \delta>0$. If $\lambda \in S$, then the system (2.19)-(2.21) either has no analytic solutions, or it has a 1-parameter family of analytic solutions. 
Proof. Since $F(c)$ is analytic in $c$ for $0 \leq c \leq 1, c_{\lambda}(r)$ is analytic in $r$ for $0 \leq r \leq 1$ and $0<\lambda<1$. We shall prove that for $\lambda \in(0,1) \backslash S$ the analytic solution of $(2.19)-(2.21)$ is given by the power series

$$
u_{\lambda}(r)=\sum_{n=0}^{\infty} \frac{r^{n}}{n !} u_{\lambda}^{(n)}(0), \quad p_{\lambda}(r)=\sum_{n=0}^{\infty} \frac{r^{n}}{n !} p_{\lambda}^{(n)}(0)
$$

where the derivatives $u_{\lambda}^{(n)}(0), p_{\lambda}^{(n)}(0)$ are computed inductively from (2.19)-(2.21), and they satisfy the inequalities

$$
\left|u_{\lambda}^{(n)}(0)\right| \leq \frac{H_{0} H^{n-1}}{n^{2}} n !, \quad\left|p_{\lambda}^{(n)}(0)\right| \leq \frac{H_{0} H^{n-1}}{n^{2}} n !
$$

for $n \geq 1$ and some positive constants $H_{0}, H$ (depending on $\lambda$ ); here $H_{0}$ is such that (4.2) holds if $n=1$, and $H$ will be specified later on. Clearly, if these assertions are proved, then the first part of the theorem follows.

We begin by formally computing the derivatives $u_{\lambda}^{(n)}(0), p_{\lambda}^{(n)}(0)$. By $(2.20)$ we have $u_{\lambda}(0)=0$, so that, by $(2.21)$,

$$
K_{P}\left(c_{\lambda}(0)\right)+\left(K_{M}\left(c_{\lambda}(0)\right)-K_{N}\left(c_{\lambda}(0)\right)\right) p_{\lambda}(0)-K_{M}\left(c_{\lambda}(0)\right) p_{\lambda}^{2}(0)=0 .
$$

It follows, by Lemma 3.1, that

$$
p_{\lambda}(0)=\alpha\left(c_{\lambda}(0)\right)=\alpha(\lambda)
$$

and then, from (2.21),

$$
u_{\lambda}^{\prime}(0)=\frac{1}{3}\left(-K_{D}(\lambda)+K_{M}(\lambda) \alpha(\lambda)\right)=\beta(\lambda) .
$$

Next we differentiate the equation (2.21) and take $r=0$. Since $c_{\lambda}^{\prime}(0)=0$, we get

$$
\gamma_{1}(0) p_{\lambda}^{\prime}(0)=0
$$

and since $\gamma_{1}(\lambda) \neq 0$,

$$
p_{\lambda}^{\prime}(0)=0
$$

Suppose we have computed $u_{\lambda}^{(m)}(0), p_{\lambda}^{(m)}(0)$ for $m=0,1, \cdots, n-1$. Multiplying (2.19) by $r$, differentiating $n$ times and taking $r=0$, we get

$$
u_{\lambda}^{(n)}(0)=-\frac{n}{n+2} k_{D}^{(n-1)}(\lambda)+\frac{n}{n+2} \sum_{m=0}^{n-1}\left(\begin{array}{c}
n-1 \\
m
\end{array}\right) k_{M}^{(m)}(\lambda) p_{\lambda}^{(n-1-m)}(0),
$$

where

$$
k_{i}^{(m)}(\lambda)=\left.\frac{d^{m}}{d r^{m}}\right|_{r=0} K_{i}\left(c_{\lambda}(r)\right), \quad i=D, M, N, P, \quad m=0,1, \cdots
$$


Similarly, by differentiating (2.21) $n$ times and taking $r=0$, we obtain

$$
\begin{aligned}
\gamma_{n}(\lambda) p_{\lambda}^{(n)}(0)= & k_{P}^{(n)}(\lambda)+\sum_{m=1}^{n}\left(\begin{array}{c}
n \\
m
\end{array}\right)\left(k_{M}^{(m)}(\lambda)-k_{N}^{(m)}(\lambda)\right) p_{\lambda}^{(n-m)}(0) \\
& -\sum_{m=1}^{n} \sum_{l=0}^{n-m}\left(\begin{array}{c}
n \\
m
\end{array}\right)\left(\begin{array}{c}
n-m \\
l
\end{array}\right) k_{M}^{(m)}(\lambda) p_{\lambda}^{(l)}(0) p_{\lambda}^{(n-m-l)}(0) \\
& -K_{M}(\lambda) \sum_{m=1}^{n-1}\left(\begin{array}{c}
n \\
m
\end{array}\right) p_{\lambda}^{(m)}(0) p_{\lambda}^{(n-m)}(0) \\
& -\sum_{m=2}^{n}\left(\begin{array}{c}
n \\
m
\end{array}\right) u_{\lambda}^{(m)}(0) p_{\lambda}^{(n-m+1)}(0) .
\end{aligned}
$$

Hence $u_{\lambda}^{(n)}(0)$ and $p_{\lambda}^{(n)}(0)$ are uniquely determined for all $n \geq 0$ provided $\lambda \notin S$.

Since $\lambda \notin S$, there exists a constant $c_{0}>0$ (depending on $\lambda$ ) such that

$$
\left|\gamma_{n}(\lambda)\right| \geq c_{0}
$$

for all $n \geq 1$. Since $c_{\lambda}(r)$ is analytic in $r$ and $K_{i}(c)$ is analytic in $c, i=D, M, N, P$, it follows that $K_{i}\left(c_{\lambda}(r)\right)$ is also analytic in $r$, which implies that

$$
\left|k_{i}^{(m)}(\lambda)\right| \leq \frac{A_{0} A^{m}}{m^{2}} m !(i=D, M, N, P, m=1,2, \cdots)
$$

for some constants $A_{0}, A$ (depending on $\lambda$ ). Using these inequalities, one can now easily prove that if (4.2) holds for all $1 \leq n \leq m$, then

$$
\left|u_{\lambda}^{(m+1)}(0)\right| \leq \frac{C H_{0} H^{m-1}}{(m+1)^{2}}(m+1) !, \quad\left|p_{\lambda}^{(m+1)}(0)\right| \leq \frac{C H_{0} H^{m-1}}{(m+1)^{2}}(m+1) !,
$$

where $C$ depends only on $A_{0}, A$ and $H_{0}$. Taking $H \geq C$, we conclude, by induction, that (4.2) holds for all $n \geq 1$.

Suppose next that $\lambda \in S$. Then there exists a positive integer $n$ such that $\gamma_{n}(\lambda)=0$. It follows that $(2.19)-(2.21)$ cannot have an analytic solution if the right-hand side of (4.9) is not equal to zero. If instead the right-hand side of (4.9) vanishes, then we can take an arbitrary value for $p_{\lambda}^{(n)}(0)$ and argue similarly as above to conclude that (2.19)-(2.21) has an analytic solution for each choice of $p_{\lambda}^{(n)}(0)$.

Remark 4.1. If $\lambda \in S$, then both of the two cases mentioned in Theorem 4.1 can occur. For instance, by (4.5) we see that if $\gamma_{1}(\lambda)=0$, then $p_{\lambda}^{\prime}(0)$ can be any real number, so that the system (2.19)-(2.21) has a 1-parameter family of analytic solutions. On the other hand, for $n=2$ the equation (4.9) reads as follows:

$$
\begin{aligned}
\gamma_{2}(\lambda) p_{\lambda}^{\prime \prime}(0) & =\left(K_{P}^{\prime}(\lambda)+\left(K_{M}^{\prime}(\lambda)-K_{N}^{\prime}(\lambda)\right) \alpha(\lambda)-K_{M}^{\prime}(\lambda) \alpha^{2}(\lambda)\right) c_{\lambda}^{\prime \prime}(0) \\
& =\left(2 K_{M}(\lambda) \alpha(\lambda)-\left(K_{M}(\lambda)-K_{N}(\lambda)\right)\right) \alpha^{\prime}(\lambda) c_{\lambda}^{\prime \prime}(0) \quad(\text { by }(3.3)-(3.5)) \\
& \left.=\sqrt{\left(K_{M}(\lambda)-K_{N}(\lambda)\right)^{2}+4 K_{M}(\lambda) K_{P}(\lambda)} \alpha^{\prime}(\lambda) c_{\lambda}^{\prime \prime}(0) \quad \text { (by }(3.2)\right)
\end{aligned}
$$

Since the right-hand side is positive for all $\lambda \in(0,1)$, the system $(2.19)-(2.21)$ cannot have analytic solutions if $\gamma_{2}(\lambda)=0$. 
The above examples show that analytic solutions do not depend continuously on $\lambda$. Therefore, by working with analytic solutions we cannot use the shooting method to find a solution of the free boundary problem (2.19)-(2.22). In the next section we shall consider general (non-analytic) solutions of (2.19)-(2.21) in a small interval $0 \leq r \leq \delta$.

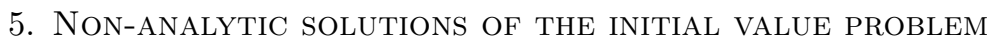

In this section we shall consider general classical solutions of the problem (2.19)(2.21). By a classical solution of $(2.19)-(2.21)$ on an interval $[0, \delta](\delta>0)$ we mean a pair of functions $p, u$ in $C[0, \delta] \cap C^{1}(0, \delta)$ that satisfy the equations (2.19), (2.21) for $0<r<\delta$, with $u(0)=0$.

We first consider solutions of (2.19)-(2.21) in the class

$$
u \in C^{1}[0, \delta], \quad p \in C^{1}[0, \delta]
$$

for some $\delta>0$. By (2.21) and (2.19), for such solutions we have

$$
p(0)=\alpha(\lambda), \quad u^{\prime}(0)=\beta(\lambda) .
$$

We shall also impose the condition

$$
p^{\prime}(0)=0 .
$$

This condition is satisfied if $p \in C^{2}[0, \delta]$ and $\gamma_{1}(\lambda) \neq 0$ (but see Theorem 5.4).

We introduce new variables $P, U$ by

$$
p(r)=\alpha(\lambda)+r P(r), \quad u(r)=r(\beta(\lambda)+U(r)) .
$$

Then

$$
\begin{gathered}
P \in C[0, \delta] \cap C^{1}(0, \delta], \quad U \in C[0, \delta] \cap C^{1}(0, \delta], \\
P(0)=0, \quad U(0)=0
\end{gathered}
$$

and

$$
\begin{gathered}
U^{\prime}(r)+\frac{3}{r} U(r)=K_{M}\left(c_{\lambda}(r)\right) P(r)+m_{\lambda}(r), \\
P^{\prime}(r)-\frac{\sigma(\lambda)}{r} P(r)=f_{\lambda}(r, P(r), U(r)),
\end{gathered}
$$

where

$$
f_{\lambda}(r, P, U)=\left(-K_{M}\left(c_{\lambda}(r)\right) P^{2}+\frac{g_{\lambda}(0)}{\beta(\lambda)} \frac{U P}{r}-\frac{g_{\lambda}(r)-g_{\lambda}(0)}{r} P+\frac{h_{\lambda}(r)}{r^{2}}\right) /(U+\beta(\lambda)),
$$

where

$$
\begin{gathered}
g_{\lambda}(r)=2 K_{M}\left(c_{\lambda}(r)\right) \alpha(\lambda)-\left(K_{M}\left(c_{\lambda}(r)\right)-K_{N}\left(c_{\lambda}(r)\right)\right), \\
h_{\lambda}(r)=K_{P}\left(c_{\lambda}(r)\right)+\left(K_{M}\left(c_{\lambda}(r)\right)-K_{N}\left(c_{\lambda}(r)\right)\right) \alpha(\lambda)-K_{M}\left(c_{\lambda}(r)\right) \alpha^{2}(\lambda) .
\end{gathered}
$$


Since the function $-K_{D}\left(c_{\lambda}(r)\right)+K_{M}\left(c_{\lambda}(r)\right) \alpha(\lambda)-3 \beta(\lambda)$ is in $C^{\infty}$ up to $r=0$ and is equal to zero at $r=0$, the function $m_{\lambda}(r)$ is in $C^{\infty}$ up to $r=0$. Similarly, the functions $\left(g_{\lambda}(r)-g_{\lambda}(0)\right) / r$ and $h_{\lambda}(r) / r^{2}$ are also in $C^{\infty}$ up to $r=0$. Furthermore,

$$
\begin{gathered}
m_{\lambda}(r)=\frac{r}{2} c_{\lambda}^{\prime \prime}(0)\left(-K_{D}^{\prime}(\lambda)+K_{M}^{\prime}(\lambda) \alpha(\lambda)\right)+O\left(r^{2}\right) \\
\frac{1}{r}\left(g_{\lambda}(r)-g_{\lambda}(0)\right)=\frac{r}{2} c_{\lambda}^{\prime \prime}(0)\left\{2 K_{M}^{\prime}(\lambda) \alpha(\lambda)-\left(K_{M}^{\prime}(\lambda)-K_{N}^{\prime}(\lambda)\right)\right\}+O\left(r^{2}\right)
\end{gathered}
$$

$$
\frac{h_{\lambda}(r)}{r^{2}}=\frac{1}{2} c_{\lambda}^{\prime \prime}(0)\left\{K_{P}^{\prime}(\lambda)+\left(K_{M}^{\prime}(\lambda)-K_{N}^{\prime}(\lambda)\right) \alpha(\lambda)-K_{M}^{\prime}(\lambda) \alpha^{2}(\lambda)\right\}+O(r) .
$$

We shall later on use the inequalities

$$
\sigma(\lambda)>-1 \text { if } \lambda \in\left(0, \lambda_{\infty}\right) ; \quad \sigma(\lambda)<-1 \text { if } \lambda \in\left(\lambda_{\infty}, 1\right) .
$$

We want to recast the differential equations for $U, P$ as integral equations. Clearly, equation (5.7) with the condition $U(0)=0$ is equivalent to the integral equation

$$
U(r)=\frac{1}{r^{3}} \int_{0}^{r} K_{M}\left(c_{\lambda}(\rho)\right) P(\rho) \rho^{3} d \rho+\frac{1}{r^{3}} \int_{0}^{r} m_{\lambda}(\rho) \rho^{3} d \rho .
$$

In order to derive an integral equation for $P$, we first introduce some new notation.

For any $\lambda \in[0,1] \backslash S$ we define inductively $\alpha_{n}(\lambda)(n \geq 0)$ and $\beta_{n}(\lambda)(n \geq 1)$ as follows:

$$
\begin{aligned}
\alpha_{n}(\lambda)= & \frac{1}{\gamma_{n}(\lambda)}\left\{k_{P}^{(n)}(\lambda)+\sum_{m=1}^{n}\left(\begin{array}{c}
n \\
m
\end{array}\right)\left(k_{M}^{(m)}(\lambda)-k_{N}^{(m)}(\lambda)\right) \alpha_{n-m}(\lambda)\right. \\
& -\sum_{m=1}^{n} \sum_{l=0}^{n-m}\left(\begin{array}{c}
n \\
m
\end{array}\right)\left(\begin{array}{c}
n-m \\
l
\end{array}\right) k_{M}^{(m)}(\lambda) \alpha_{l}(\lambda) \alpha_{n-m-l}(\lambda) \\
& \left.-K_{M}(\lambda) \sum_{m=1}^{n-1}\left(\begin{array}{c}
n \\
m
\end{array}\right) \alpha_{m}(\lambda) \alpha_{n-m}(\lambda)-\sum_{m=2}^{n}\left(\begin{array}{c}
n \\
m
\end{array}\right) \beta_{m}(\lambda) \alpha_{n-m+1}(\lambda)\right\}, \\
& \beta_{n+1}(\lambda)=-\frac{n+1}{n+3} k_{D}^{(n)}(\lambda)+\frac{n+1}{n+3} \sum_{m=0}^{n}\left(\begin{array}{c}
n \\
m
\end{array}\right) k_{M}^{(m)}(\lambda) \alpha_{n-m}(\lambda),
\end{aligned}
$$

for $n=1,2, \cdots$, where $k_{i}^{(n)}(\lambda)$ are as in (4.8). Note that

$$
\alpha_{n}(\lambda)=p_{\lambda}^{(n)}(0), \quad \beta_{n}(\lambda)=u_{\lambda}^{(n)}(0) \quad\left(\beta_{0}(\lambda)=0\right),
$$

for $0<\lambda<1$, where $\left(p_{\lambda}, u_{\lambda}\right)$ is the unique analytic solution of (2.19)-(2.21) (cf. $(4.7),(4.9))$. Note also, by $(5.3)$ and the fact that $c_{\lambda}^{\prime}(0)=0$, that

$$
\alpha_{1}(\lambda)=0, \quad \beta_{2}(\lambda)=0 .
$$

We also define

$$
\mu_{n}(\lambda) \equiv \frac{\alpha_{n+2}(\lambda) \gamma_{n+2}(\lambda)}{(n+2) ! \beta(\lambda)}=\frac{\alpha_{n+2}(\lambda)}{(n+2) !}(n+1-\sigma(\lambda)) \quad(n=0,1,2, \cdots)
$$


For $\lambda \in S, \gamma_{1}(\lambda) \neq 0$, if $n$ is the nonnegative integer such that $\gamma_{n+2}(\lambda)=0$ (i.e., $\sigma(\lambda)=n+1)$, then $\alpha_{i}(\lambda)(0 \leq i \leq n+1), \beta_{i}(\lambda)(1 \leq i \leq n+2)$ and $\mu_{i}(\lambda)$ $(0 \leq i \leq n-1)$ are still well-defined by $(5.19)-(5.21)$. We also define $\mu_{n}(\lambda)$ by

$$
\begin{aligned}
\mu_{n}(\lambda)= & \frac{1}{(n+2) ! \beta(\lambda)}\left\{k_{P}^{(n+2)}(\lambda)+\sum_{m=1}^{n+2}\left(\begin{array}{c}
n+2 \\
m
\end{array}\right)\left(k_{M}^{(m)}(\lambda)-k_{N}^{(m)}(\lambda)\right) \alpha_{n-m+2}(\lambda)\right. \\
& -\sum_{m=1}^{n+2} \sum_{l=0}^{n-m+2}\left(\begin{array}{c}
n+2 \\
m
\end{array}\right)\left(\begin{array}{c}
n+2-m \\
l
\end{array}\right) k_{M}^{(m)}(\lambda) \alpha_{l}(\lambda) \alpha_{n-m-l+2}(\lambda) \\
& -K_{M}(\lambda) \sum_{m=1}^{n+1}\left(\begin{array}{c}
n+2 \\
m
\end{array}\right) \alpha_{m}(\lambda) \alpha_{n-m+2}(\lambda) \\
& \left.-\sum_{m=2}^{n+2}\left(\begin{array}{c}
n+2 \\
m
\end{array}\right) \beta_{m}(\lambda) \alpha_{n-m+3}(\lambda)\right\} .
\end{aligned}
$$

This is consistent with (5.21), since $\gamma_{n+2}(\lambda)$ appears as a denominator in the definition of $\alpha_{n+2}(\lambda)$ (see (5.19)).

The following lemma gives an equivalent integral form of the equation (5.8).

Lemma 5.1. Let $P(r), U(r) \in C[0, \delta] \cap C^{1}(0, \delta]$ be a solution of (5.6)-(5.8) and assume that $\lambda \neq \lambda_{\infty}$. Then, if $\delta$ is sufficiently small, the following assertions hold:

(1) If either $\beta(\lambda)>0$ or $\gamma_{1}(\lambda) \leq 0(\Longleftrightarrow \sigma(\lambda) \leq 0)$, then $(P(r), U(r))$ satisfies the equation

$$
P(r)=r^{\sigma(\lambda)} \int_{0}^{r} f_{\lambda}(\rho, P(\rho), U(\rho)) \rho^{-\sigma(\lambda)} d \rho .
$$

(2) If $\gamma_{1}(\lambda)>0$ and $\gamma_{2}(\lambda)<0(\Longleftrightarrow 0<\sigma(\lambda)<1)$, then the limit

$$
\omega=\lim _{r \rightarrow 0} r^{-\sigma(\lambda)} P(r)
$$

exists, and $(P(r), U(r))$ satisfies the equation

$$
P(r)=\omega r^{\sigma(\lambda)}+r^{\sigma(\lambda)} \int_{0}^{r} f_{\lambda}(\rho, P(\rho), U(\rho)) \rho^{-\sigma(\lambda)} d \rho .
$$

(3) If $\gamma_{2}(\lambda)=0(\Longleftrightarrow \sigma(\lambda)=1)$, then the limit

$$
\omega=\lim _{r \rightarrow 0} r^{-1}\left(P(r)-\mu_{0}(\lambda) r \log r\right)
$$

exists, and $(P(r), U(r))$ satisfies the equation

$$
P(r)=\omega r+\mu_{0}(\lambda) r \log r+r \int_{0}^{r}\left(f_{\lambda}(\rho, P(\rho), U(\rho))-\mu_{0}(\lambda)\right) \rho^{-1} d \rho .
$$

(4) In general, if for some integer $n \geq 2$ we have $\gamma_{n}(\lambda)>0, \gamma_{n+1}(\lambda)<0$ $(\Longleftrightarrow n-1<\sigma(\lambda)<n)$, then the limit

$$
\omega=\lim _{r \rightarrow 0} r^{-\sigma(\lambda)}\left(P(r)-\sum_{m=1}^{n-1} \frac{\alpha_{m+1}(\lambda)}{(m+1) !} r^{m}\right)
$$


exists, and $(P(r), U(r))$ satisfies the equation

$$
\begin{aligned}
P(r)= & \omega r^{\sigma(\lambda)}+\sum_{m=1}^{n-1} \frac{\alpha_{m+1}(\lambda)}{(m+1) !} r^{m} \\
& +r^{\sigma(\lambda)} \int_{0}^{r}\left(f_{\lambda}(\rho, P(\rho), U(\rho))-\sum_{m=0}^{n-2} \mu_{m}(\lambda) \rho^{m}\right) \rho^{-\sigma(\lambda)} d \rho .
\end{aligned}
$$

If instead $\gamma_{n+1}(\lambda)=0(\Longleftrightarrow \sigma(\lambda)=n)$, then the limit

$$
\omega=\lim _{r \rightarrow 0} r^{-n}\left(P(r)-\sum_{m=1}^{n-1} \frac{\alpha_{m+1}(\lambda)}{(m+1) !} r^{m}-\mu_{n-1}(\lambda) r^{n} \log r\right)
$$

exists, and $(P(r), U(r))$ satisfies the equation

$$
\begin{aligned}
P(r)= & \omega r^{n}+\sum_{m=1}^{n-1} \frac{\alpha_{m+1}(\lambda)}{(m+1) !} r^{m}+\mu_{n-1}(\lambda) r^{n} \log r \\
& +r^{n} \int_{0}^{r}\left(f_{\lambda}(\rho, P(\rho), U(\rho))-\sum_{m=0}^{n-2} \mu_{m}(\lambda) \rho^{m}\right) \rho^{-n} d \rho .
\end{aligned}
$$

Conversely, if $P(r), U(r) \in C[0, \delta]$ and they satisfy the coupled system of equations (5.17) and one of the equations (5.23)-(5.27) (in accordance with the corresponding condition on $\lambda)$, then $(P(r), U(r))$ is a solution of the problem (5.5)-(5.8).

We need the following lemma:

Lemma 5.2. (1) Let $P(r), U(r) \in C[0, \delta]$ and suppose that they satisfy the equation (5.17). Suppose further that, for some integer $n \geq 1$,

$$
P(r)=\sum_{m=1}^{n} \frac{\alpha_{m}(\lambda)}{m !} r^{m-1}+O\left(r^{n}\right) \text { as } r \rightarrow 0
$$

Then

$$
U(r)=\sum_{m=2}^{n+1} \frac{\beta_{m}(\lambda)}{m !} r^{m-1}+O\left(r^{n+1}\right) \text { as } r \rightarrow 0 .
$$

(2) Let $f_{\lambda}(r, P(r), U(r))$ be the function given by (5.11) and $\lambda \neq \lambda_{\infty}$. If $P(r)$, $U(r)$ have the expansions (5.28) and (5.29), then

$$
f_{\lambda}(r, P(r), U(r))=\sum_{m=0}^{n-1} \mu_{m}(\lambda) r^{m}+O\left(r^{n}\right) \text { as } r \rightarrow 0 .
$$

Proof. Since the functions $K_{M}\left(c_{\lambda}(r)\right)$ and $m_{\lambda}(r)$ are in $C^{\infty}$ up to $r=0$, we have

$$
K_{M}\left(c_{\lambda}(r)\right)=\sum_{m=0}^{n} \frac{1}{m !} k_{M}^{(m)}(\lambda) r^{m}+O\left(r^{n+1}\right),
$$

as $r \rightarrow 0$, and a similar expansion holds for $m_{\lambda}(r)$. Substituting these expressions and (5.28) into (5.17) yields (5.29). Next, since $\left(g_{\lambda}(r)-g_{\lambda}(0)\right) / r$ and $h_{\lambda}(r) / r^{2}$ are also in $C^{\infty}$ up to $r=0$ and $U(0)+\beta(\lambda) \neq 0$, it is clear that $f_{\lambda}(r, P(r), U(r))$ has 
an expansion

$$
f_{\lambda}(r, P(r), U(r))=\sum_{m=0}^{n-1} a_{m}(\lambda) r^{m}+O\left(r^{n}\right) \text { as } r \rightarrow 0 .
$$

The proof that $a_{m}(\lambda)=\mu_{m}(\lambda)$ is given in the Appendix ( $\left.\S 12\right)$.

Proof of Lemma 5.1. Consider Case (1) first. It is clear that $f_{\lambda}(r, P(r), U(r))$ is bounded for $r \in(0, \delta]$ if $\delta$ is sufficiently small. Thus multiplying (5.8) by $r^{-\sigma(\lambda)}$ and integrating over the arbitrary interval $[0, r]$ immediately yields the equation (5.23).

Consider Case (2) next. Since $P(r) \in C[0, \delta]$, there exists a constant $C$ (depending on $\lambda$ and $\delta$ ) such that

$$
|P(r)| \leq C \quad \text { for } 0 \leq r \leq \delta .
$$

In the sequel we shall use $C$ to denote various positive constants (depending on $\lambda$ and $\delta$ ). By (5.17) it follows that

$$
|U(r)| \leq C r \quad \text { for } 0 \leq r \leq \delta .
$$

Thus $U(r) / r$ is bounded, and

$$
|U(r)+\beta(\lambda)| \geq C>0 \quad \text { for } 0 \leq r \leq \delta
$$

if $\delta$ is sufficiently small. Consequently,

$$
\left|f_{\lambda}(r, P(r), U(r))\right| \leq C \quad \text { for } 0<r \leq \delta
$$

and, since $0<\sigma(\lambda)<1, f_{\lambda}(r, P(r), U(r)) r^{-\sigma(\lambda)}$ is integrable on $(0, \delta]$. We now multiply (5.8) by $r^{-\sigma(\lambda)}$ and integrate over the interval [s,r], for arbitrary $0<s<$ $r \leq \delta$, to get

$$
r^{-\sigma(\lambda)} P(r)-s^{-\sigma(\lambda)} P(s)=\int_{s}^{r} f_{\lambda}(\rho, P(\rho), U(\rho)) \rho^{-\sigma(\lambda)} d \rho .
$$

Since the right-hand side converges to zero as $r \rightarrow 0$, the limit

$$
\omega=\lim _{r \rightarrow 0} r^{-\sigma(\lambda)} P(r)
$$

exists. Multiplying (5.34) by $r^{-\sigma(\lambda)}$ and letting $s \rightarrow 0$, (5.24) follows.

Consider Case (3). We rewrite (5.34) in the form

$$
r^{-1} P(r)-s^{-1} P(s)=\int_{s}^{r}\left(f_{\lambda}(\rho, P(\rho), U(\rho))-\mu_{0}(\lambda)\right) \rho^{-1} d \rho+\mu_{0}(\lambda)(\log r-\log s) .
$$

We claim that

$$
|P(r)| \leq C r \log \frac{1}{r} \quad \text { for } 0<r \leq \delta .
$$

Indeed, as before, (5.31)-(5.34) hold (with $\sigma(\lambda)=1$ ), so that

$$
\left|\delta^{-1} P(\delta)-r^{-1} P(r)\right| \leq \int_{r}^{\delta}\left|f_{\lambda}(\rho, P(\rho), U(\rho))\right| \rho^{-1} d \rho \leq C(\log \delta-\log r)
$$

for $0<r \leq \delta$, and (5.36) follows. Substituting (5.36) into (5.17), we find that

$$
|U(r)| \leq C r^{2} \log \frac{1}{r} \quad \text { for } 0<r \leq \delta
$$


It follows that

$$
\begin{aligned}
& \left|f_{\lambda}(r, P(r), U(r))-\mu_{0}(\lambda)\right| \\
& \quad \leq C|P(r)|^{2}+C\left|\frac{U(r) P(r)}{r}\right|+C r|P(r)|+\left|\frac{h_{\lambda}(r)}{r^{2}(U(r)+\beta(\lambda))}-\mu_{0}(\lambda)\right| \\
& \quad \leq C r^{2} \log ^{2} r+C r^{2} \log ^{2} r+C r^{2} \log \frac{1}{r}+C r \leq C r
\end{aligned}
$$

so that $\int_{0}^{\delta}\left|f_{\lambda}(\rho, P(\rho), U(\rho))-\mu_{0}(\lambda)\right| \rho^{-1} d \rho<\infty$. Consequently, by (5.35), the limit

$$
\omega=\lim _{r \rightarrow 0} r^{-1}\left(P(r)-\mu_{0}(\lambda) r \log r\right)
$$

exists, and letting $s \rightarrow 0$ in (5.35) we see that (5.25) holds.

Consider now the general case $\gamma_{n}(\lambda)>0, \gamma_{n+1}(\lambda)<0$, namely, $n-1<\sigma(\lambda)<n$, where $n \geq 2$. As before, (5.31)-(5.34) hold, so that

$$
\begin{aligned}
\left|\delta^{-\sigma(\lambda)} P(\delta)-r^{-\sigma(\lambda)} P(r)\right| & \leq \int_{r}^{\delta}\left|f_{\lambda}(\rho, P(\rho), U(\rho))\right| \rho^{-\sigma(\lambda)} d \rho \\
& \leq C\left(\delta^{1-\sigma(\lambda)}-r^{1-\sigma(\lambda)}\right)
\end{aligned}
$$

for $0<r \leq \delta$. It follows that

$$
|P(r)| \leq C r \quad \text { for } 0 \leq r \leq \delta .
$$

By (5.17) we further deduce that

$$
|U(r)| \leq C r^{2} \quad \text { for } 0 \leq r \leq \delta
$$

As in the derivation of (5.38), these estimates allow us to prove that

$$
\left|f_{\lambda}(r, P(r), U(r))-\mu_{0}(\lambda)\right| \leq C r \quad \text { for } 0<r \leq \delta .
$$

We rewrite (5.34) in the form

$$
\begin{aligned}
& r^{-\sigma(\lambda)} P(r)-s^{-\sigma(\lambda)} P(s) \\
& \quad=\int_{s}^{r}\left(f_{\lambda}(\rho, P(\rho), U(\rho))-\mu_{0}(\lambda)\right) \rho^{-\sigma(\lambda)} d \rho+\frac{\mu_{0}(\lambda)}{1-\sigma(\lambda)}\left(r^{1-\sigma(\lambda)}-s^{1-\sigma(\lambda)}\right) .
\end{aligned}
$$

If $1<\sigma(\lambda)<2$, then $\int_{0}^{\delta}\left|f_{\lambda}(\rho, P(\rho), U(\rho))-\mu_{0}(\lambda)\right| \rho^{-\sigma(\lambda)} d \rho<\infty$, and the desired assertion follows by an argument similar to that used in the case $\sigma(\lambda)=1$. It remains to consider the case $\sigma(\lambda)>2$. By (5.42) and (5.41) it follows that

$$
\begin{aligned}
& \left|\left(\delta^{-\sigma(\lambda)} P(\delta)-r^{-\sigma(\lambda)} P(r)\right)-\frac{\mu_{0}(\lambda)}{1-\sigma(\lambda)}\left(\delta^{1-\sigma(\lambda)}-r^{1-\sigma(\lambda)}\right)\right| \\
& \quad \leq \int_{r}^{\delta}\left|f_{\lambda}(\rho, P(\rho), U(\rho))-\mu_{0}(\lambda)\right| \rho^{-\sigma(\lambda)} d \rho \\
& \quad \leq C\left|\delta^{2-\sigma(\lambda)}-r^{2-\sigma(\lambda)}\right|
\end{aligned}
$$

for $0<r \leq \delta$. Hence we have

$$
\left|P(r)-\frac{\mu_{0}(\lambda)}{1-\sigma(\lambda)} r\right| \leq C r^{2} \quad \text { for } 0 \leq r \leq \delta,
$$

or equivalently (by (5.21)),

$$
\left|P(r)-\frac{1}{2} \alpha_{2}(\lambda) r\right| \leq C r^{2} \quad \text { for } 0 \leq r \leq \delta
$$


It follows, by Lemma $5.2(1)$, that

$$
\left|U(r)-\frac{1}{6} \beta_{3}(\lambda) r^{2}\right| \leq C r^{3} \quad \text { for } 0 \leq r \leq \delta,
$$

and by Lemma $5.2(2)$, that

$$
\left|f_{\lambda}(r, P(r), U(r))-\mu_{0}(\lambda)-\mu_{1}(\lambda) r\right| \leq C r^{2} \quad \text { for } 0<r \leq \delta .
$$

Repeating the above bootstrap argument step-by-step, we arrive at the estimate

$$
\left|f_{\lambda}(r, P(r), U(r))-\sum_{m=0}^{n-2} \mu_{m}(\lambda) r^{m}\right| \leq C r^{n-1} \quad \text { for } 0<r \leq \delta,
$$

so that

$$
\int_{0}^{\delta}\left|f_{\lambda}(\rho, P(\rho), U(\rho))-\sum_{m=0}^{n-2} \mu_{m}(\lambda) \rho^{m}\right| \rho^{-\sigma(\lambda)} d \rho<\infty .
$$

Since for any $0<s<r \leq \delta$ we have

$$
\begin{gathered}
\left(r^{-\sigma(\lambda)} P(r)-\sum_{m=1}^{n-1} \frac{\alpha_{m+1}(\lambda)}{(m+1) !} r^{m-\sigma(\lambda)}\right)-\left(s^{-\sigma(\lambda)} P(s)-\sum_{m=1}^{n-1} \frac{\alpha_{m+1}(\lambda)}{(m+1) !} s^{m-\sigma(\lambda)}\right) \\
=\int_{s}^{r}\left(f_{\lambda}(\rho, P(\rho), U(\rho))-\sum_{m=0}^{n-2} \mu_{m}(\lambda) \rho^{m}\right) \rho^{-\sigma(\lambda)} d \rho,
\end{gathered}
$$

it follows that the limit

$$
\omega=\lim _{r \rightarrow 0} r^{-\sigma(\lambda)}\left(P(r)-\sum_{m=1}^{n-1} \frac{\alpha_{m+1}(\lambda)}{(m+1) !} r^{m}\right)
$$

exists and, by a similar argument as before, (5.26) holds.

The argument for the case $\gamma_{n+1}(\lambda)=0$ (i.e., $\sigma(\lambda)=n$ ) is similar.

Finally, it is rather immediate to verify that if $(P(r), U(r))$ satisfies $(5.17)$ and one of the equations (5.23)-(5.27), then it also satisfies (5.6)-(5.8).

The first main result of this section is as follows:

Theorem 5.3. Let $\lambda \neq \lambda_{\infty}$. Then the following hold:

(1) If either $\beta(\lambda)>0$ or $\gamma_{1}(\lambda) \leq 0(\Longleftrightarrow \sigma(\lambda) \leq 0)$, then the system $(2.19)-(2.21)$ has a unique local solution satisfying conditions (5.1)-(5.3).

(2) If $\beta(\lambda)<0$ and $\gamma_{1}(\lambda)>0(\Longleftrightarrow \sigma(\lambda)>0)$, then the system $(2.19)-(2.21)$ has a 1-parameter family of local solutions satisfying conditions (5.1)-(5.3). More precisely, we have:

(i) If $\gamma_{n}(\lambda)>0, \gamma_{n+1}(\lambda)<0(\Longleftrightarrow n-1<\sigma(\lambda)<n)$ for some positive integer $n$, then for every real number $\omega$ the above system has a unique local solution satisfying the following conditions:

$$
\begin{gathered}
p(r)=\sum_{m=0}^{n} \frac{\alpha_{m}(\lambda)}{m !} r^{m}+\omega r^{1+\sigma(\lambda)}+O\left(r^{n+1}\right) \text { as } r \rightarrow 0, \\
u(r)=\sum_{m=1}^{n+1} \frac{\beta_{m}(\lambda)}{m !} r^{m}+\frac{\omega K_{M}(\lambda)}{4+\sigma(\lambda)} r^{2+\sigma(\lambda)}+O\left(r^{n+2}\right) \text { as } r \rightarrow 0 ;
\end{gathered}
$$

moreover, these are all the solutions of (2.19)-(2.21) satisfying (5.1)-(5.3). 
(ii) If $\gamma_{n+1}(\lambda)=0(\Longleftrightarrow \sigma(\lambda)=n)$ for some positive integer $n$, then for every real number $\omega$ the above system has a unique local solution satisfying the following conditions:

$$
\begin{array}{r}
p(r)=\sum_{m=0}^{n} \frac{\alpha_{m}(\lambda)}{m !} r^{m}+\mu_{n-1}(\lambda) r^{n+1} \log r+\omega r^{n+1}+O\left(r^{n+2} \log r\right) \text { as } r \rightarrow 0, \\
u(r)=\sum_{m=1}^{n+1} \frac{\beta_{m}(\lambda)}{m !} r^{m}+\frac{\mu_{n-1}(\lambda) K_{M}(\lambda)}{n+4} r^{n+2} \log r+\tilde{\omega} r^{n+2}+O\left(r^{n+3} \log r\right) \\
\text { as } r \rightarrow 0,
\end{array}
$$

where

$$
\begin{aligned}
\tilde{\omega}= & \frac{K_{M}(\lambda)}{n+4}\left(\omega-\frac{\mu_{n-1}(\lambda)}{n+4}\right) \\
& +\frac{1}{(n+4)(n+1) !}\left(-k_{D}^{(n+1)}(\lambda)+\sum_{m=0}^{n}\left(\begin{array}{c}
n+1 \\
m
\end{array}\right) k_{M}^{(n+1)}(\lambda) \alpha_{m}(\lambda)\right)
\end{aligned}
$$

moreover, these are all the solutions of (2.19)-(2.21) satisfying (5.1)-(5.3).

Proof. By Lemma 5.1, we only need to prove that the system of equations formed by (5.17) coupled with one of the equations (5.23)-(5.27) has a unique solution in the class $C[0, \delta]$, for some small $\delta>0$. We shall use the contraction mapping principle to prove this.

The argument for the system of equations (5.17), (5.23) is simple, and we omit it. Since (5.24) and (5.25) are special situations of (5.26) and (5.27), respectively, it suffices to consider the systems (5.17), (5.26) and (5.17), (5.27).

We first consider the system $(5.17),(5.26)$. For small $\delta>0$, we denote by $\tilde{X}_{\delta}$ the set of continuous functions $(P(r), U(r))$ defined on $[0, \delta]$ satisfying the following conditions:

$$
\begin{gathered}
\left|P(r)-\sum_{m=2}^{n} \frac{\alpha_{m}(\lambda)}{m !} r^{m-1}\right| \leq(1+|\omega|) r^{\sigma(\lambda)} \quad \text { for } 0 \leq r \leq \delta \\
\left|U(r)-\sum_{m=3}^{n+1} \frac{\beta_{m}(\lambda)}{m !} r^{m-1}\right| \leq\left(1+\left|\frac{\omega K_{M}(\lambda)}{4+\sigma(\lambda)}\right|\right) r^{1+\sigma(\lambda)} \quad \text { for } 0 \leq r \leq \delta .
\end{gathered}
$$

As in the proof of Lemma 5.2, one can show that if $\delta$ is small enough, then

$$
\left|f_{\lambda}(r, P(r), U(r))-\sum_{m=0}^{n-2} \mu_{m}(\lambda) r^{m}\right| \leq C r^{n-1}
$$

for $0<r \leq \delta$ and for all $(P(r), U(r)) \in \tilde{X}_{\delta}$. Here and later on we use $C$ to denote various positive constants depending only on $\delta, \lambda, \omega$ and $n$ (but independent of the specific functions $(P, U))$. We now introduce a metric space $\left(X_{\delta}, d\right)$ as follows: The set $X_{\delta}$ consists of continuous functions $(P(r), U(r))$ defined on $[0, \delta]$, satisfying the inequalities

$$
\left|P(r)-\sum_{m=2}^{n} \frac{\alpha_{m}(\lambda)}{m !} r^{m-1}-\omega r^{\sigma(\lambda)}\right| \leq M_{1} r^{n}
$$




$$
\left|U(r)-\sum_{m=3}^{n+1} \frac{\beta_{m}(\lambda)}{m !} r^{m-1}-\frac{\omega K_{M}(\lambda)}{4+\sigma(\lambda)} r^{1+\sigma(\lambda)}\right| \leq M_{2} r^{n+1},
$$

for $0 \leq r \leq \delta$, where $M_{1}=C /(n-\sigma(\lambda)), C$ being the constant in (5.49), and $M_{2}$ is another constant to be specified later on. The distance function $d$ is defined by

$$
d\left(\left(P_{1}, U_{1}\right),\left(P_{2}, U_{2}\right)\right)=\sup _{0<r \leq \delta} \frac{\left|P_{1}(r)-P_{2}(r)\right|}{r^{\sigma(\lambda)}}+\sup _{0<r \leq \delta} \frac{\left|U_{1}(r)-U_{2}(r)\right|}{r^{1+\sigma(\lambda)-\theta}},
$$

where $0<\theta<1$ is an arbitrarily chosen number. Clearly, $\left(X_{\delta}, d\right)$ is a complete metric space, and $X_{\delta} \subset \tilde{X}_{\delta}$ if $\delta$ is sufficiently small.

Consider the mapping $\mathcal{F}:(P, U) \rightarrow(\tilde{P}, \tilde{U})$ defined by

$$
\begin{aligned}
\tilde{P}(r)= & \omega r^{\sigma(\lambda)}+\sum_{m=2}^{n} \frac{\alpha_{m}(\lambda)}{m !} r^{m-1} \\
& +r^{\sigma(\lambda)} \int_{0}^{r}\left(f_{\lambda}(\rho, P(\rho), U(\rho))-\sum_{m=0}^{n-2} \mu_{m}(\lambda) \rho^{m}\right) \rho^{-\sigma(\lambda)} d \rho, \\
\tilde{U}(r)= & \frac{1}{r^{3}} \int_{0}^{r} K_{M}\left(c_{\lambda}(\rho)\right) P(\rho) \rho^{3} d \rho+\frac{1}{r^{3}} \int_{0}^{r} m_{\lambda}(\rho) \rho^{3} d \rho,
\end{aligned}
$$

where $(P, U) \in \tilde{X}_{\delta}$. We claim that $(\tilde{P}, \tilde{U}) \in X_{\delta}$. Indeed, using (5.49), it follows immediately that $\tilde{P}(r)$ satisfies (5.50). To see that $\tilde{U}(r)$ satisfies (5.51) we set

$$
\begin{aligned}
\Delta_{\lambda}(r) \equiv & \frac{1}{r^{3}} \int_{0}^{r} K_{M}\left(c_{\lambda}(\rho)\right)\left(\sum_{m=2}^{n} \frac{\alpha_{m}(\lambda)}{m !} \rho^{m-1}+\omega \rho^{\sigma(\lambda)}\right) \rho^{3} d \rho \\
& +\frac{1}{r^{3}} \int_{0}^{r} m_{\lambda}(\rho) \rho^{3} d \rho-\sum_{m=3}^{n+1} \frac{\beta_{m}(\lambda)}{m !} r^{m-1}-\frac{\omega K_{M}(\lambda)}{4+\sigma(\lambda)} r^{1+\sigma(\lambda)} .
\end{aligned}
$$

If we replace $K_{M}\left(c_{\lambda}(\rho)\right)$ and $m_{\lambda}(\rho)$ (from (5.10)) by their Taylor expansions of order up to $n$ and recall the definition of $\beta_{m}(\lambda)$ from (5.20), we easily find that all the powers of order $<n+1$ cancel out. Hence, there exists constant $C_{1}>0$ such that

$$
\left|\Delta_{\lambda}(r)\right| \leq C_{1} r^{n+1}
$$

for $0<r \leq \delta$. It follows that

$$
\begin{aligned}
& \left|\tilde{U}(r)-\sum_{m=3}^{n+1} \frac{\beta_{m}(\lambda)}{m !} r^{m-1}-\frac{\omega K_{M}(\lambda)}{4+\sigma(\lambda)} r^{1+\sigma(\lambda)}\right| \\
& \quad \leq \frac{1}{r^{3}} \int_{0}^{r}\left|K_{M}\left(c_{\lambda}(\rho)\right)\right|\left|P(\rho)-\sum_{m=2}^{n} \frac{\alpha_{m}(\lambda)}{m !} \rho^{m-1}-\omega \rho^{\sigma(\lambda)}\right| \rho^{3} d \rho+\left|\Delta_{\lambda}(r)\right| \\
& \quad \leq\left(\frac{C_{2} M}{n+4}+C_{1}\right) r^{n+1}
\end{aligned}
$$

for $0<r \leq \delta$, where $C_{2}$ denotes the maximum of $\left|K_{M}\left(c_{\lambda}(r)\right)\right|$ for $0 \leq r \leq \delta$. Taking $M_{2}$ to be the constant on the right-hand side of the last inequality, we see that $\tilde{U}(r)$ satisfies $(5.51)$. Thus $(\tilde{P}, \tilde{U}) \in X_{\delta}$ and $\mathcal{F}$ maps $\tilde{X}_{\delta}$ into $X_{\delta}$.

Next, we take any $\left(P_{1}, U_{1}\right),\left(P_{2}, U_{2}\right)$ in $X_{\delta}$ and set

$$
\left(\tilde{P}_{i}, \tilde{U}_{i}\right)=\mathcal{F}\left(P_{i}, U_{i}\right), \quad i=1,2 .
$$


It is easy to verify that for any chosen $\theta \in(0,1)$,

$$
\begin{aligned}
& \sup _{0<r \leq \delta}\left|f_{\lambda}\left(r, P_{1}(r), U_{1}(r)\right)-f_{\lambda}\left(r, P_{2}(r), U_{2}(r)\right)\right| / r^{1+\sigma(\lambda)-\theta} \\
& \quad \leq C d\left(\left(P_{1}, U_{1}\right),\left(P_{2}, U_{2}\right)\right) .
\end{aligned}
$$

This enables us to deduce that

$$
\sup _{0<r \leq \delta}\left|\tilde{P}_{1}(r)-\tilde{P}_{2}(r)\right| / r^{\sigma(\lambda)} \leq C \delta^{2-\theta} d\left(\left(P_{1}, U_{1}\right),\left(P_{2}, U_{2}\right)\right) .
$$

It is also readily seen that

$$
\sup _{0<r \leq \delta}\left|\tilde{U}_{1}(r)-\tilde{U}_{2}(r)\right| / r^{1+\sigma(\lambda)-\theta} \leq C \delta^{\theta} d\left(\left(P_{1}, U_{1}\right),\left(P_{2}, U_{2}\right)\right),
$$

so that

$$
d\left(\left(\tilde{P}_{1}, \tilde{U}_{1}\right),\left(\tilde{P}_{2}, \tilde{U}_{2}\right)\right) \leq C \delta^{\theta} d\left(\left(P_{1}, U_{1}\right),\left(P_{2}, U_{2}\right)\right) .
$$

Thus $\mathcal{F}$ is a contraction mapping in $X_{\delta}$, provided $\delta$ is sufficiently small, and consequently, $\mathcal{F}$ has a unique fixed point in $X_{\delta}$.

The proof in the case $\gamma_{n+1}(\lambda)=0(\Longleftrightarrow \sigma(\lambda)=n)$ is similar.

Remark 5.1. The analytic solutions constructed in $\S 4$ for $\lambda \in(0,1) \backslash S$ coincide with the solutions (5.43), (5.44) corresponding to $\omega=0$.

From Theorem 5.3 we see that in the case $\gamma_{1}(\lambda) \leq 0$, the solution $(p, u)$ of (2.19)-(2.21) satisfying $p, u \in C^{1}[0, \delta]$ and $p^{\prime}(0)=0$ is unique. If we weaken these regularity conditions, we can also get a 1-parameter family of solutions, as in the case $\gamma_{1}(\lambda)>0$ and $\beta(\lambda)<0$. More precisely, we have the following result:

Theorem 5.4. (1) Suppose that $\gamma_{1}(\lambda)=0(\Longleftrightarrow \sigma(\lambda)=0)$. Then for any $\omega \in \mathbf{R}$, the problem (2.19)-(2.21) has a unique solution $(p(r), u(r))$ for $0 \leq r \leq \delta$, for some small $\delta>0$, satisfying

$$
p, u \in C^{1}[0, \delta] \text { and } p^{\prime}(0)=\omega .
$$

(2) Suppose that $\gamma_{1}(\lambda)<0(\Longleftrightarrow-1<\sigma(\lambda)<0)$. Then for any $\omega \in \mathbf{R}$, the problem (2.19)-(2.21) has a unique solution $(p(r), u(r))$ for $0 \leq r \leq \delta$, for some $\delta>0$, satisfying the following conditions:

$$
\begin{gathered}
p \in C[0, \delta] \cap C^{1}(0, \delta], \quad u \in C^{1}[0, \delta] ; \\
p(r)=\alpha(\lambda)+\omega r^{1+\sigma(\lambda)}+O(r) \text { as } r \rightarrow 0, \\
u(r)=\beta(\lambda) r+\frac{\omega K_{M}(\lambda)}{4+\sigma(\lambda)} r^{2+\sigma(\lambda)}+O\left(r^{2}\right) \text { as } r \rightarrow 0 .
\end{gathered}
$$

Proof. Consider the change of variables

$$
p(r)=\alpha(\lambda)+r^{1+\sigma(\lambda)} P(r), \quad u(r)=r(\beta(\lambda)+U(r)),
$$

where $P, U \in C[0, \delta] \cap C^{1}(0, \delta]$ and they satisfy the initial conditions

$$
P(0)=\omega, \quad U(0)=0 .
$$

One can readily verify that, under the transformation (5.57), the equations (2.19) and (2.21) are respectively changed into the equations

$$
\begin{gathered}
U^{\prime}(r)+\frac{3}{r} U(r)=r^{\sigma(\lambda)} K_{M}\left(c_{\lambda}(r)\right) P(r)+m_{\lambda}(r), \\
P^{\prime}(r)=\tilde{f}_{\lambda}(r, P(r), U(r)),
\end{gathered}
$$


where $m_{\lambda}(r)$ is as in (5.10), and $\tilde{f}_{\lambda}(r, P, U)$ is given by

$$
\begin{aligned}
\tilde{f}_{\lambda}(r, P, U)=( & -r^{\sigma(\lambda)} K_{M}\left(c_{\lambda}(r)\right) P^{2}+\frac{g_{\lambda}(0)}{\beta(\lambda)} \frac{U P}{r} \\
& \left.-\frac{g_{\lambda}(r)-g_{\lambda}(0)}{r} P+\frac{h_{\lambda}(r)}{r^{2+\sigma(\lambda)}}\right) /(\beta(\lambda)+U),
\end{aligned}
$$

where $g_{\lambda}(r), h_{\lambda}(r)$ are as in (5.12) and (5.13), respectively. Since $-1<\sigma(\lambda) \leq 0$, the term $r^{\sigma(\lambda)} K_{M}\left(c_{\lambda}(r)\right)$ in (5.59) and $\tilde{f}_{\lambda}$ are integrable. Also, the term $U P / r$ is integrable if $U(r)$ converges to 0 at an appropriate rate as $r \rightarrow 0$. This enables us to transform the problem (5.58)-(5.60) into the following system of integral equations:

$$
\begin{gathered}
U(r)=\frac{1}{r^{3}} \int_{0}^{r} K_{M}\left(c_{\lambda}(\rho)\right) P(\rho) \rho^{3+\sigma(\lambda)} d \rho+\frac{1}{r^{3}} \int_{0}^{r} m_{\lambda}(\rho) \rho^{3} d \rho, \\
P(r)=\omega+\int_{0}^{r} \tilde{f}_{\lambda}(\rho, P(\rho), U(\rho)) d \rho .
\end{gathered}
$$

Using the contraction mapping principle as in the proof of Theorem 5.3, we can prove that there exists $\delta>0$ such that the system (5.61), (5.62) has a unique continuous solution $(P(r), U(r))$ for $0 \leq r \leq \delta$, satisfying

$$
|P(r)| \leq M_{1}, \quad|U(r)| \leq M_{2} r^{1+\sigma(\lambda)} \quad(0 \leq r \leq \delta)
$$

for some constants $M_{1}$ and $M_{2}$. Hence the desired result follows.

Later in $\S 8$ we shall see that the solutions of (2.19)-(2.21) constructed in this section are unique also within the class of nonnegative weak solutions (see Theorem 8.1).

\section{Continuous dependence of solutions on the Parameters}

By the results obtained in the previous section, for every $0<\lambda<\lambda_{\infty}$ the problem (2.19)-(2.21) has a continuum of solutions depending on a real parameter $\omega$. From (5.23)-(5.27) and (5.62) we see, at least heuristically, that, for a fixed $\omega$, the solution depends continuously on $\lambda$ for $0<\lambda<\lambda_{\infty}, \lambda \notin S_{1}$, where

$$
\begin{aligned}
S_{1} & =\left\{\lambda \in\left(0, \lambda_{\infty}\right): \gamma_{n}(\lambda)=0 \text { for some } n \geq 2\right\} \\
& =\left\{\lambda \in\left(0, \lambda_{\infty}\right): \sigma(\lambda)=m \text { for some integer } m \geq 1\right\} .
\end{aligned}
$$

However, from the structure of the solutions (in Theorem 5.3) it is clear that as $\lambda \rightarrow \bar{\lambda}$ for some $\bar{\lambda} \in S_{1}$, the solution does not have a limit. The main purpose of this section is to show that we can reparameterize the solutions for $0<\lambda<\lambda_{\infty}$ by a new parameter $\psi$ in such a way that when $\omega=\omega(\lambda, \psi)$ the solution will depend continuously on $\lambda$ for all $0<\lambda<\lambda_{\infty}$, as well as on $\psi$.

We introduce the set

$$
A=\left\{\lambda \in\left(0, \lambda_{\infty}\right): \gamma_{1}(\lambda)>0\right\}=\left\{\lambda \in\left(0, \lambda_{\infty}\right): \sigma(\lambda)>0\right\},
$$

which is clearly an open set containing $S_{1}$. By Lemma 3.3, the following two situations may occur: (1) $A$ is a single interval $\left(\lambda_{0}, \lambda_{\infty}\right)$, where $\lambda_{0}$ is either equal to zero (in the case $\sigma(\lambda)>0$ for all $0<\lambda<\lambda_{\infty}$ ) or equal to the unique positive root of the equation $\sigma(\lambda)=0$ (in the case that $\sigma(\lambda)$ changes sign only once); (2) $A$ is a union of finitely many disjoint open intervals, one of which has the form $\left(\lambda_{0}, \lambda_{\infty}\right)$, where $\lambda_{0}$ is the largest root of the equation $\sigma(\lambda)=0$. For any $\lambda \in A$ and $\omega \in \mathbf{R}$, we denote by $(P(r ; \lambda, \omega), U(r ; \lambda, \omega))$ the solution of the system consisting of $(5.17)$ and 
one of the equations (5.23)-(5.27) (in accordance with the corresponding condition on $\lambda)$, and for any $\lambda \in\left(0, \lambda_{\infty}\right) \backslash A$ we denote by $(P(r ; \lambda, \omega), U(r ; \lambda, \omega))$ the solution of the system of equations (5.61) and (5.62).

Consider Case (1) first. By Lemma 3.3, the set $S_{1}$ consists of a finite number of points $\bar{\lambda}_{1}, \bar{\lambda}_{2}, \cdots, \bar{\lambda}_{m}$, which are the roots of the equations $\gamma_{2}(\lambda)=0, \gamma_{3}(\lambda)=0$, $\cdots, \gamma_{n_{0}-1}(\lambda)=0$, and the sequence $\left\{\lambda_{n}\right\}_{n \geq n_{0}}$ as described by Lemma 3.3. We rearrange the union of the two sets of these $\lambda$ 's as a monotone increasing sequence and denote it as

$$
\bar{\lambda}_{1}, \bar{\lambda}_{2} \cdots, \bar{\lambda}_{n}, \cdots .
$$

By Lemma 3.3 we know that $\lim _{n \rightarrow \infty} \bar{\lambda}_{n}=\lambda_{\infty}$. For each integer $n \geq 1$ we take a small number $\varepsilon_{n}>0$ such that all the intervals

$$
\left[\bar{\lambda}_{1}-\varepsilon_{1}, \bar{\lambda}_{1}+\varepsilon_{1}\right], \quad\left[\bar{\lambda}_{2}-\varepsilon_{2}, \bar{\lambda}_{2}+\varepsilon_{2}\right], \cdots,\left[\bar{\lambda}_{n}-\varepsilon_{n}, \bar{\lambda}_{n}+\varepsilon_{n}\right], \cdots
$$

are contained in $\left(\lambda_{0}, \lambda_{\infty}\right)$ and do not intersect each other. Let $m_{n}=\sigma\left(\bar{\lambda}_{n}\right)$. Clearly, if $\sigma(\lambda)$ is monotone increasing, then $m_{n+1}=m_{n}+1$; for general $\sigma(\lambda)$ we only have $\left|m_{n+1}-m_{n}\right| \leq 1$. We introduce, for every integer $n \geq 1$, a function $\omega_{n}(\lambda, \psi)$ $\left(\lambda \in\left[\bar{\lambda}_{n}-\varepsilon_{n}, \overline{\bar{\lambda}}_{n}+\varepsilon_{n}\right], \psi \in \mathbf{R}\right)$ as follows:

$$
\omega_{n}(\lambda, \psi)=\left\{\begin{array}{l}
\psi \text { if } \lambda=\bar{\lambda}_{n}, \\
\psi-\left(\mu_{m_{n}-1}(\lambda) /\left(m_{n}-\sigma(\lambda)\right)\right) \quad \text { if } 0<\left|\lambda-\bar{\lambda}_{n}\right| \leq \varepsilon_{n} .
\end{array}\right.
$$

We arbitrarily take a continuous function $\omega_{n, n+1}(\lambda, \psi)$ defined for $\bar{\lambda}_{n}+\varepsilon_{n} \leq \lambda \leq$ $\bar{\lambda}_{n+1}-\varepsilon_{n+1}$ and $\psi \in \mathbf{R}$ and monotone increasing in $\psi$ such that

$$
\begin{aligned}
\omega_{n, n+1}\left(\bar{\lambda}_{n}+\varepsilon_{n}, \psi\right) & =\omega_{n}\left(\bar{\lambda}_{n}+\varepsilon_{n}, \psi\right), \\
\omega_{n, n+1}\left(\bar{\lambda}_{n+1}-\varepsilon_{n+1}, \psi\right) & =\omega_{n+1}\left(\bar{\lambda}_{n+1}-\varepsilon_{n+1}, \psi\right) .
\end{aligned}
$$

For $\lambda_{0}<\lambda \leq \bar{\lambda}_{1}-\varepsilon_{1}$ we take

$$
\omega_{0,1}(\lambda, \psi)=\psi-\left(\mu_{n_{1}-1}(\lambda) /\left(n_{1}-\sigma(\lambda)\right)\right)
$$

Finally, in case $\lambda_{0}>0$ we define

$$
\omega_{0}(\lambda, \psi)=\psi-\mu_{0}(\lambda) \quad\left(0<\lambda \leq \lambda_{0}, \quad \psi \in \mathbf{R}\right) .
$$

Now define, for all $0<\lambda<\lambda_{\infty}$ and $\psi \in \mathbf{R}$, a function $\omega(\lambda, \psi)$ by

$$
\omega(\lambda, \psi)=\left\{\begin{array}{l}
\omega_{0}(\lambda, \psi) \text { if } 0<\lambda \leq \lambda_{0} \\
\omega_{0,1}(\lambda, \psi) \text { if } \lambda_{0}<\lambda \leq \bar{\lambda}_{1}-\varepsilon_{1} \\
\omega_{n}(\lambda, \psi) \text { if } \bar{\lambda}_{n}-\varepsilon_{n}<\lambda<\bar{\lambda}_{n}+\varepsilon_{n}, \quad n=1,2, \cdots \\
\omega_{n, n+1}(\lambda, \psi) \text { if } \bar{\lambda}_{n}+\varepsilon_{n} \leq \lambda \leq \bar{\lambda}_{n+1}-\varepsilon_{n+1}, \quad n=1,2, \cdots
\end{array}\right.
$$

It follows that $\omega(\lambda, \psi)$ is continuous for $\lambda \in\left(0, \lambda_{\infty}\right) \backslash S_{1}$ and $\psi \in \mathbf{R}$, and is monotone increasing in $\psi$. Notice however that $\omega(\lambda, \psi)$ is not continuous at $\bar{\lambda} \in S_{1}$, since

$$
\lim _{\lambda \rightarrow \bar{\lambda}} \omega(\lambda, \psi)= \pm \infty
$$

Consider Case (2) next. We write

$$
A=\left(\lambda_{01}, \lambda_{02}\right) \cup\left(\lambda_{03}, \lambda_{04}\right) \cup \cdots \cup\left(\lambda_{02 k-1}, \lambda_{02 k}\right) \cup\left(\lambda_{0}, \lambda_{\infty}\right) .
$$

In the last interval we can define the function $\omega(\lambda, \psi)$ in a way similar to (6.6). Every interval of the form $\left(\lambda_{02 i-1}, \lambda_{02 i}\right)$ contains at most a finite number of points from $S_{1}$. We write these points in increasing order, say,

$$
\bar{\lambda}_{i 1}, \bar{\lambda}_{i 2}, \cdots, \bar{\lambda}_{i m}
$$


As before, for every integer $1 \leq j \leq m$ we take a small positive number $\varepsilon_{j}$ such that all the intervals

$$
\left[\bar{\lambda}_{i 1}-\varepsilon_{1}, \bar{\lambda}_{i 1}+\varepsilon_{1}\right],\left[\bar{\lambda}_{i 2}-\varepsilon_{2}, \bar{\lambda}_{i 2}+\varepsilon_{2}\right], \cdots,\left[\bar{\lambda}_{i m}-\varepsilon_{m}, \bar{\lambda}_{i m}+\varepsilon_{m}\right]
$$

are contained in $\left(\lambda_{02 i-1}, \lambda_{02 i}\right)$ and do not intersect each other. We then define $\omega(\lambda, \psi)$ for $\lambda \in\left[\bar{\lambda}_{i j}-\varepsilon_{j}, \bar{\lambda}_{i j}+\varepsilon_{j}\right]$ and $\psi \in \mathbf{R}(j=1,2, \cdots, m)$ similarly as in (6.2), and, for every $1 \leq j \leq m-1$, we arbitrarily define the values of $\omega(\lambda, \psi)$ for $\lambda \in$ $\left(\bar{\lambda}_{i j}+\varepsilon_{j}, \bar{\lambda}_{i j+1}-\varepsilon_{j+1}\right)$ and $\psi \in \mathbf{R}$ such that it is continuous on

$$
\left[\bar{\lambda}_{i j}+\varepsilon_{j}, \bar{\lambda}_{i j+1}-\varepsilon_{j+1}\right] \times \mathbf{R}
$$

and monotone increasing in $\psi$. For $\lambda \in\left(\lambda_{02 i-1}, \bar{\lambda}_{i 1}-\varepsilon_{1}\right) \cup\left(\bar{\lambda}_{i m}+\varepsilon_{m}, \lambda_{02 i}\right)$, we define $\omega(\lambda, \psi)$ similarly as in (6.4). Finally, in the complementary set of $A$, which consists of the union $\left(0, \lambda_{01}\right] \cup\left[\lambda_{02}, \lambda_{03}\right] \cup\left[\lambda_{04}, \lambda_{05}\right] \cup \cdots \cup\left[\lambda_{02 k}, \lambda_{0}\right]$, we define

$$
\omega(\lambda, \psi)=\psi-\mu_{0}(\lambda) \text { for } \psi \in \mathbf{R} .
$$

Clearly, the function $\omega(\lambda, \psi)\left(\lambda \in\left(0, \lambda_{\infty}\right), \psi \in \mathbf{R}\right)$ defined in this way is continuous for all $\lambda \in\left(0, \lambda_{\infty}\right) \backslash S_{1}$ and $\psi \in \mathbf{R}$, but not for $\lambda \in S_{1}$, and is monotone increasing in $\psi$.

Having introduced the function $\omega(\lambda, \psi)\left(0<\lambda<\lambda_{\infty}, \psi \in \mathbf{R}\right)$, we now define

$$
P_{\lambda \psi}(r)=P(r ; \lambda, \omega(\lambda, \psi)), \quad U_{\lambda \psi}(r)=U(r ; \lambda, \omega(\lambda, \psi))
$$

and

$$
p_{\lambda \psi}(r)=\alpha(\lambda)+r P_{\lambda \psi}(r), \quad u_{\lambda \psi}(r)=r\left(\beta(\lambda)+U_{\lambda \psi}(r)\right)
$$

for all $0<\lambda<\lambda_{\infty}$ and $\psi \in \mathbf{R}$.

The main result of this section is the following:

Theorem 6.1. For any $0<\bar{\lambda}<\lambda_{\infty}$ and $\bar{\psi} \in \mathbf{R}$, there exists a corresponding $\delta>0$ such that for any $(\lambda, \psi)$ in a neighborhood of $(\bar{\lambda}, \bar{\psi})$, the solution $\left(p_{\lambda \psi}(r), u_{\lambda \psi}(r)\right)$ exists for all $r \in[0, \delta]$, and

$$
\lim _{(\lambda, \psi) \rightarrow(\bar{\lambda}, \bar{\psi})} p_{\lambda \psi}(r)=p_{\bar{\lambda} \bar{\psi}}(r), \quad \lim _{(\lambda, \psi) \rightarrow(\bar{\lambda}, \bar{\psi})} u_{\lambda \psi}(r)=u_{\bar{\lambda} \bar{\psi}}(r)
$$

uniformly for $r \in[0, \delta]$.

We shall only give the proof for the case $(\bar{\lambda}, \bar{\psi}) \in S_{1} \times R$; the proof for the case $\bar{\lambda} \in\left(0, \lambda_{\infty}\right) \backslash S_{1}$ (in particular, the case where $\left.\sigma(\bar{\lambda})=0\right)$ is similar but simpler. We need the following lemma:

Lemma 6.2 Let $\bar{\lambda} \in S_{1}, \bar{\psi} \in \mathbf{R}$. Then there exist positive constants $\delta, C$ such that for $0<|\lambda-\bar{\lambda}| \ll 1$ and $|\psi-\bar{\psi}| \ll 1$, the solution $\left(P_{\lambda \psi}(r), U_{\lambda \psi}(r)\right)$ exists for all $0 \leq r \leq \delta$ and

$$
\left|P_{\lambda \psi}(r)-\psi r^{\sigma(\lambda)}-\sum_{m=2}^{n} \frac{\alpha_{m}(\lambda)}{m !} r^{m-1}-\mu_{n-1}(\lambda) \frac{r^{n}-r^{\sigma(\lambda)}}{n-\sigma(\lambda)}\right| \leq C r^{n+1},
$$

where $n=\sigma(\bar{\lambda})$; the constants $\delta, C$ are independent of $\lambda$ and $\psi$.

Proof. Notice that since $\bar{\lambda} \in S_{1}, n=\sigma(\bar{\lambda})$ is an integer $\geq 1$. In the following, when we say " $\lambda$ near $\bar{\lambda}$ ", we mean that $0<|\lambda-\bar{\lambda}| \ll 1$, and similarly for " $\psi$ near $\bar{\psi}$ ". 
Since $P_{\lambda \psi}(r)=P(r ; \lambda, \omega(\lambda, \psi))$ and $\omega(\lambda, \psi)=\psi-\mu_{n-1}(\lambda) /(n-\sigma(\lambda))$ for $\lambda$ near $\bar{\lambda}$, we see that $P_{\lambda \psi}(r)$ satisfies the equation

$$
\begin{aligned}
P(r)= & \psi r^{\sigma(\lambda)}+\sum_{m=2}^{n} \frac{\alpha_{m}(\lambda)}{m !} r^{m-1}+\mu_{n-1}(\lambda) \frac{r^{n}-r^{\sigma(\lambda)}}{n-\sigma(\lambda)} \\
& +r^{\sigma(\lambda)} \int_{0}^{r}\left(f_{\lambda}(\rho, P(\rho), U(\rho))-\sum_{m=0}^{n-1} \mu_{m}(\lambda) \rho^{m}\right) \rho^{-\sigma(\lambda)} d \rho .
\end{aligned}
$$

As in the proof of Theorem 5.3, we then have

$$
P(r)=\omega r^{\sigma(\lambda)}+\sum_{m=2}^{n} \frac{\alpha_{m}(\lambda)}{m !} r^{m-1}+\mu_{n-1}(\lambda) \frac{r^{n}-r^{\sigma(\lambda)}}{n-\sigma(\lambda)}+O\left(r^{n^{\prime}}\right)
$$

as $r \rightarrow 0$, where $n^{\prime}=n$ for $n-1<\sigma(\lambda)<n$, and $n^{\prime}=n+1$ for $n<\sigma(\lambda)<n+1$. Thus for $\lambda$ near $\bar{\lambda}$ and for all $\psi \in \mathbf{R}$, the function

$$
\Delta_{\lambda \psi}(r)=\sup _{0<\rho \leq r}\left|P_{\lambda \psi}(\rho)-\sum_{m=2}^{n} \frac{\alpha_{m}(\lambda)}{m !} \rho^{m-1}\right| \rho^{-n+\frac{1}{2}}
$$

converges to zero as $r \rightarrow 0$. Let $\delta_{0}>0$ be a fixed small number, and let $\delta_{\lambda \psi}$ be the largest number in $\left(0, \delta_{0}\right]$ such that the solution of the system $(6.11),(5.17)$ exists for $0 \leq r<\delta_{\lambda \psi}$. We can clearly write, for $0<\rho \leq r<\delta_{\lambda \psi}$,

$$
\left|P_{\lambda \psi}(\rho)-\sum_{m=2}^{n} \frac{\alpha_{m}(\lambda)}{m !} \rho^{m-1}\right| \leq \rho^{n-\frac{1}{2}} \Delta_{\lambda \psi}(r)
$$

By (5.17) we further have

$$
\begin{aligned}
\left|U_{\lambda \psi}(\rho)-\sum_{m=3}^{n+1} \frac{\beta_{m}(\lambda)}{m !} \rho^{m-1}\right| & \leq C \rho^{n+\frac{1}{2}} \Delta_{\lambda \psi}(\rho)+C \rho^{n+1} \\
& \leq C \rho^{n+\frac{1}{2}} \Delta_{\lambda \psi}(r)+C \rho^{n+1}
\end{aligned}
$$

for $0<\rho \leq r<\delta_{\lambda \psi}$; here and in what follows we use $C$ to denote various positive constants independent of $\lambda$ and $\psi$. We introduce the functions

$$
v_{\lambda \psi}(r)=P_{\lambda \psi}(r)-\sum_{m=2}^{n} \frac{\alpha_{m}(\lambda)}{m !} r^{m-1}, \quad w_{\lambda \psi}(r)=U_{\lambda \psi}(r)-\sum_{m=3}^{n+1} \frac{\beta_{m}(\lambda)}{m !} r^{m-1}
$$

so that

$$
\begin{gathered}
P_{\lambda \psi}(r)=\sum_{m=2}^{n} \frac{\alpha_{m}(\lambda)}{m !} r^{m-1}+v_{\lambda \psi}(r), \\
U_{\lambda \psi}(r)+\beta(\lambda)=\sum_{m=1}^{n+1} \frac{\beta_{m}(\lambda)}{m !} r^{m-1}+w_{\lambda \psi}(r)
\end{gathered}
$$

(recall that $\left.\beta_{1}(\lambda)=\beta(\lambda), \beta_{2}(\lambda)=0\right)$. By $(6.14),(6.15)$,

$$
\left|v_{\lambda \psi}(\rho)\right| \leq \rho^{n-\frac{1}{2}} \Delta_{\lambda \psi}(r), \quad\left|w_{\lambda \psi}(\rho)\right| \leq C \rho^{n+\frac{1}{2}} \Delta_{\lambda \psi}(r)+C \rho^{n+1}
$$


for $0<\rho \leq r<\delta_{\lambda \psi}$. It follows (see the Appendix) that

$$
\begin{aligned}
& \left(U_{\lambda \psi}(\rho)+\beta(\lambda)\right)\left(f_{\lambda}\left(\rho, P_{\lambda \psi}(\rho), U_{\lambda \psi}(\rho)\right)-\sum_{m=0}^{n-1} \mu_{m}(\lambda) \rho^{m}\right) \\
& =\rho^{n} y_{\lambda \psi}(\rho)+z_{1 \lambda \psi}(\rho) w_{\lambda \psi}(\rho)+\rho z_{2 \lambda \psi}(\rho) v_{\lambda \psi}(\rho)-K_{M}\left(c_{\lambda}(\rho)\right) v_{\lambda \psi}^{2}(\rho),
\end{aligned}
$$

where

$$
\left|y_{\lambda \psi}(\rho)\right| \leq C, \quad\left|z_{1 \lambda \psi}(\rho)\right| \leq C, \quad\left|z_{2 \lambda \psi}(\rho)\right| \leq C
$$

for $0<\rho<\delta_{\lambda \psi}$ and for $\lambda, \psi$ respectively near $\bar{\lambda}$ and $\bar{\psi}$. Hence,

$$
\begin{gathered}
\left|U_{\lambda \psi}(\rho)+\beta(\lambda)\right|\left|f_{\lambda}\left(\rho, P_{\lambda \psi}(\rho), U_{\lambda \psi}(\rho)\right)-\sum_{m=0}^{n-1} \mu_{m}(\lambda) \rho^{m}\right| \\
\leq C \rho^{2 n-1} \Delta_{\lambda \psi}^{2}(r)+C \rho^{n+\frac{1}{2}} \Delta_{\lambda \psi}(r)+C \rho^{n} .
\end{gathered}
$$

By the second inequality in (6.18) it follows that

$$
\begin{aligned}
\left|U_{\lambda \psi}(\rho)+\beta(\lambda)\right| & \geq|\beta(\lambda)|-\left|U_{\lambda \psi}(\rho)\right| \\
& \geq|\beta(\lambda)|-\left(C r^{2}+C r^{n+\frac{1}{2}} \Delta_{\lambda \psi}(r)\right) .
\end{aligned}
$$

Since $\lim _{r \rightarrow 0} \Delta_{\lambda \psi}(r)=0$ and $\beta(\lambda) \neq 0$, we infer that for every $(\lambda, \psi)$ near $(\bar{\lambda}, \bar{\psi})$, there exists a corresponding positive number $\tilde{\delta}_{\lambda \psi} \leq \delta_{\lambda \psi}$ such that for $0<r<\tilde{\delta}_{\lambda \psi}$,

$$
C r^{2}+C r^{\frac{3}{2}} \Delta_{\lambda \psi}(r) \leq \frac{1}{2}|\beta(\lambda)| .
$$

Thus

$$
\left|U_{\lambda \psi}(\rho)+\beta(\lambda)\right| \geq \frac{1}{2}|\beta(\lambda)| \geq C>0
$$

for $0<\rho<\tilde{\delta}_{\lambda \psi}$ and for $\lambda, \psi$ respectively near $\bar{\lambda}$ and $\bar{\psi}$. In what follows we use the same notation $\tilde{\delta}_{\lambda \psi}$ to denote the supremum of all $\tilde{\delta}_{\lambda \psi}$ such that (6.21) holds for $0<r<\tilde{\delta}_{\lambda \psi}$. Substituting (6.22) into (6.20), we get

$$
\begin{aligned}
& \left|f_{\lambda}\left(\rho, P_{\lambda \psi}(\rho), U_{\lambda \psi}(\rho)\right)-\sum_{m=0}^{n-1} \mu_{m}(\lambda) \rho^{m}\right| \\
& \quad \leq C \rho^{2 n-1} \Delta_{\lambda \psi}^{2}(r)+C \rho^{n+\frac{1}{2}} \Delta_{\lambda \psi}(r)+C \rho^{n}
\end{aligned}
$$

and then, by (6.11),

$$
\begin{aligned}
& \left|P_{\lambda \psi}(r)-\psi r^{\sigma(\lambda)}-\sum_{m=2}^{n} \frac{\alpha_{m}(\lambda)}{m !} r^{m-1}-\mu_{n-1}(\lambda) \frac{r^{n}-r^{\sigma(\lambda)}}{n-\sigma(\lambda)}\right| \\
& \leq C r^{2 n} \Delta_{\lambda \psi}^{2}(r)+C r^{n+\frac{3}{2}} \Delta_{\lambda \psi}(r)+C r^{n+1}
\end{aligned}
$$

for $0<r<\tilde{\delta}_{\lambda \psi}$. The term with the coefficient $\mu_{n-1}(\lambda)$ can be estimated from the inequality

$$
\left|\frac{r^{n}-r^{\sigma}}{n-\sigma}\right| \leq C r^{\frac{1}{2}}
$$


(for $r>0, \sigma \geq n-1 / 4$ ), and this leads to

$$
\begin{aligned}
& \left|P_{\lambda \psi}(\rho)-\sum_{m=2}^{n} \frac{\alpha_{m}(\lambda)}{m !} \rho^{m-1}\right| \rho^{-n+\frac{1}{2}} \\
& \quad \leq C \rho^{n+\frac{1}{2}} \Delta_{\lambda \psi}^{2}(\rho)+C \rho^{2} \Delta_{\lambda \psi}(\rho)+C \rho^{\frac{3}{2}}+C \rho^{\frac{1}{4}} \\
& \quad \leq C \rho^{\frac{3}{2}} \Delta_{\lambda \psi}^{2}(r)+C \rho^{\frac{1}{4}}
\end{aligned}
$$

for $0<\rho \leq r<\tilde{\delta}_{\lambda \psi}$, where $\lambda$ is sufficiently near $\bar{\lambda}$ such that $|\sigma(\lambda)-n|<1 / 4$, and $\psi$ is near $\bar{\psi}$. By (6.13), it then follows that, for $0<r<\tilde{\delta}_{\lambda \psi}$ and for $\lambda$, $\psi$ respectively near $\bar{\lambda}$ and $\bar{\psi}$,

$$
\Delta_{\lambda \psi}(r) \leq C r^{\frac{3}{2}} \Delta_{\lambda \psi}^{2}(r)+C r^{\frac{1}{4}}
$$

Thus, if $0<r<\tilde{\delta}_{\lambda \psi}$ and

$$
4 C r^{\frac{3}{2}} \cdot C r^{\frac{1}{4}}<1
$$

then

$$
\Delta_{\lambda \psi}(r) \leq \frac{1}{2 C r^{\frac{3}{2}}}\left(1-\sqrt{1-4 C r^{\frac{3}{2}} \cdot C r^{\frac{1}{4}}}\right)<2 C r^{\frac{1}{4}}
$$

We now replace the term $\Delta_{\lambda \psi}(r)$ in $(6.21)$ by the upper bound obtained in $(6.26)$ and consider the inequality

$$
C r^{2}+C r^{\frac{3}{2}} \cdot 2 C r^{\frac{1}{4}} \leq \frac{1}{2}|\beta(\lambda)|
$$

Since $|\beta(\lambda)|$ has a positive lower bound for $\lambda$ near $\bar{\lambda}$ and the left-hand side converges to zero as $r \rightarrow 0$, we can find $\delta \in\left(0, \delta_{0}\right)$ small enough so that $(6.27)$ holds for all $0<r \leq \delta$ and $\lambda$ near $\bar{\lambda}$. By taking $\delta$ sufficiently small we may assume that (6.25) also holds for $0<r \leq \delta$. We now use the maximality of $\tilde{\delta}_{\lambda \psi}$ and $\delta_{\lambda \psi}$ to prove that

$$
\delta \leq \tilde{\delta}_{\lambda \psi}\left(\leq \delta_{\lambda \psi}\right)
$$

for $\lambda, \psi$ near $\bar{\lambda}$ and $\bar{\psi}$, respectively. Indeed, we have either $\tilde{\delta}_{\lambda \psi}=\delta_{\lambda \psi}$ or $\tilde{\delta}_{\lambda \psi}<\delta_{\lambda \psi}$. Suppose first that $\tilde{\delta}_{\lambda \psi}=\delta_{\lambda \psi}$. If $\delta_{\lambda \psi}<\delta$, then, by (6.22), (6.23) and (6.26), $P_{\lambda \psi}(r)$ and, consequently, $U_{\lambda \psi}(r)$ are bounded for $0 \leq r \leq \delta_{\lambda \psi}$, and $U_{\lambda \psi}(r)+$ $\beta(\lambda)$ stays away from zero uniformly for $0 \leq r \leq \delta_{\lambda \psi}$. This allows us to extend $\left(P_{\lambda \psi}(r), U_{\lambda \psi}(r)\right)$ beyond $r=\delta_{\lambda \psi}$ as the solution of a regular ODE system, which contradicts the maximality of $\delta_{\lambda \psi}$. Hence $\delta \leq \delta_{\lambda \psi}$ holds in the case where $\tilde{\delta}_{\lambda \psi}=$ $\delta_{\lambda \psi}$. Suppose next that $\tilde{\delta}_{\lambda \psi}<\delta_{\lambda \psi}$. Then by the maximality of $\tilde{\delta}_{\lambda \psi}$, the equality in (6.21) must hold for $r=\tilde{\delta}_{\lambda \psi}$. Since the second inequality in (6.26) holds also for $r=\min \left\{\delta, \tilde{\delta}_{\lambda \psi}\right\}$, it follows, by (6.27), that $\delta<\tilde{\delta}_{\lambda \psi}$. Having thus completed the proof that $\delta \leq \delta_{\lambda \psi}$, the estimate (6.10) follows from (6.23) and (6.26).

Proof of Theorem 6.1. We shall only consider the case $\bar{\lambda} \in S_{1}$; the case $\bar{\lambda} \in$ $\left(0, \lambda_{\infty}\right) \backslash S_{1}$ can be treated similarly.

We first prove that for $\delta$ as in Lemma 6.2,

$$
\lim _{(\lambda, \psi) \rightarrow(\bar{\lambda}, \bar{\psi})} P_{\lambda \psi}(r)=P_{\bar{\lambda} \bar{\psi}}(r), \quad \lim _{(\lambda, \psi) \rightarrow(\bar{\lambda}, \bar{\psi})} U_{\lambda \psi}(r)=U_{\bar{\lambda} \bar{\psi}}(r)
$$

uniformly for all $r \in[0, \delta]$.

From Lemma 6.2 we see that

$$
\left|P_{\lambda \psi}(r)\right| \leq C r^{\frac{3}{4}}
$$


for $0 \leq r \leq \delta$ and $(\lambda, \psi)$ near $(\bar{\lambda}, \bar{\psi})$, which further implies, by (5.17), that

$$
\left|U_{\lambda \psi}(r)\right| \leq C r^{\frac{7}{4}}
$$

for $0 \leq r \leq \delta$ and $(\lambda, \psi)$ near $(\bar{\lambda}, \bar{\psi})$. It follows that

$$
\left|f_{\lambda}\left(r, P_{\lambda \psi}(r), U_{\lambda \psi}(r)\right)\right| \leq C
$$

for $0 \leq r \leq \delta$ and $(\lambda, \psi)$ near $(\bar{\lambda}, \bar{\psi})$. Using these estimates in (5.7), (5.8), we obtain

$$
\begin{gathered}
\left|U_{\lambda \psi}^{\prime}(r)\right| \leq C, \\
\left|P_{\lambda \psi}^{\prime}(r)\right| \leq C r^{-\frac{1}{4}}+C .
\end{gathered}
$$

By (6.32), (6.33) and (6.29), it follows that the family of functions

$$
\left\{\left(U_{\lambda \psi}(r), P_{\lambda \psi}(r)\right): 0<|\lambda-\bar{\lambda}| \ll 1,|\psi-\bar{\psi}| \ll 1\right\}
$$

is equicontinuous and uniformly bounded for $0 \leq r \leq \delta$. We now assert that (6.34)

$$
\lim _{(\lambda, \psi) \rightarrow(\bar{\lambda}, \bar{\psi})} \max _{0 \leq r \leq \delta}\left|P_{\lambda \psi}(r)-P_{\bar{\lambda} \bar{\psi}}(r)\right|=0, \quad \lim _{(\lambda, \psi) \rightarrow(\bar{\lambda}, \bar{\psi})} \max _{0 \leq r \leq \delta}\left|U_{\lambda \psi}(r)-U_{\bar{\lambda} \bar{\psi}}(r)\right|=0 .
$$

Indeed, otherwise there would exist a number $\varepsilon>0$ and a sequence $\left(\bar{\lambda}_{m}, \bar{\psi}_{m}\right)$ $(m=1,2, \cdots)$, converging to $(\bar{\lambda}, \bar{\psi})$, such that

$$
\max _{0 \leq r \leq \delta}\left|P_{\bar{\lambda}_{m} \bar{\psi}_{m}}(r)-P_{\bar{\lambda} \bar{\psi}}(r)\right|+\max _{0 \leq r \leq \delta}\left|U_{\bar{\lambda}_{m} \bar{\psi}_{m}}(r)-U_{\bar{\lambda} \bar{\psi}}(r)\right| \geq \varepsilon
$$

for all $m$. Since $\left\{P_{\bar{\lambda}_{m} \bar{\psi}_{m}}(r)\right\}$ and $\left\{U_{\bar{\lambda}_{m} \bar{\psi}_{m}}(r)\right\}$ are both equicontinuous and uniformly bounded for $0 \leq r \leq \varepsilon$, there exists a subsequence of $\left\{\left(\bar{\lambda}_{m}, \bar{\psi}_{m}\right)\right\}$, which for simplicity is again denoted by $\left\{\left(\bar{\lambda}_{m}, \bar{\psi}_{m}\right)\right\}$, such that $P_{\bar{\lambda}_{m} \bar{\psi}_{m}}(r)$ and $U_{\bar{\lambda}_{m} \bar{\psi}_{m}}(r)$ converge uniformly, in $[0, \delta]$, to some functions $P(r)$ and $U(r)$, respectively. Taking $(\lambda, \psi)=\left(\bar{\lambda}_{m}, \bar{\psi}_{m}\right)$ in $(5.17),(6.11)$ and using the fact that

$$
\lim _{\sigma \rightarrow n}\left(r^{n}-r^{\sigma}\right) /(n-\sigma)=r^{n} \log r
$$

and the Lebesgue dominated convergence theorem, we conclude, as $m \rightarrow \infty$, that $P(r), U(r)$ form a solution for the system of (5.17), (5.27). Since the solution of the system $(5.17),(5.27)$ is unique, we get

$$
P(r)=P_{\bar{\lambda} \bar{\psi}}(r), \quad U(r)=U_{\bar{\lambda} \bar{\psi}}(r),
$$

a contradiction to (6.35). Hence (6.34) holds. By (6.34) and (6.8), (6.9) easily follows.

\section{Global properties of the solutions}

In this section we study the global properties of solutions of the free boundary problem (2.19)-(2.22) and the initial value problem (2.19)-(2.21).

We first consider the global properties of solutions of the free boundary problem (2.19)-(2.22).

Theorem 7.1. Let $\left(p, u, R_{\lambda}\right)$ be a solution of the free boundary problem (2.19)(2.22) with $p(r), u(r)$ in $C^{1}\left[0, R_{\lambda}\right]$. Then

(1) $p^{\prime}(r)>0$ for $0<r<R_{\lambda}$;

(2) $0<p(r)<1$ for $0 \leq r<R_{\lambda}, p\left(R_{\lambda}\right)=1$;

(3) $\frac{d}{d r}\left(u^{\prime}(r)+\frac{2}{r} u(r)\right)>0$ for $0<r<R_{\lambda}$;

(4) $u(r)<0$ for $0<r<R_{\lambda}$;

(5) $u^{\prime}(0)<0, u^{\prime}\left(R_{\lambda}\right)>0$. 
Proof. First we note that the assumptions that $p, u \in C^{1}\left[0, R_{\lambda}\right]$ and $u(0)=0$ imply, by (2.19), that $u \in C^{2}\left[0, R_{\lambda}\right]$.

We begin by proving that $p^{\prime}(r) \geq 0$ for all $0 \leq r \leq R_{\lambda}$. Assume that this assertion is false, namely, there exists an $r_{0} \in\left(0, R_{\lambda}\right)$ such that $p^{\prime}\left(r_{0}\right)<0$. Denote by $\left(r_{1}, r_{2}\right)$ the maximal interval containing $r_{0}$ such that $p^{\prime}(r)<0$ for all $r \in\left(r_{1}, r_{2}\right)$. Then either $r_{1}=0$ or $0<r_{1}<r_{0}$ and $p^{\prime}\left(r_{1}\right)=0$. In both situations we get $u\left(r_{1}\right) p^{\prime}\left(r_{1}\right)=0$. This implies, by (2.21) and Lemma 3.1, that

$$
p\left(r_{1}\right)=\alpha\left(c_{\lambda}\left(r_{1}\right)\right) \text {. }
$$

Similarly, we have either $r_{2}=R_{\lambda}$, or $r_{0}<r_{2}<R_{\lambda}$ and $p^{\prime}\left(r_{2}\right)=0$. In both situations we get $u\left(r_{2}\right) p^{\prime}\left(r_{2}\right)=0$, so that

$$
p\left(r_{2}\right)=\alpha\left(c_{\lambda}\left(r_{2}\right)\right) .
$$

Since $p^{\prime}(r)<0$ for $r \in\left(r_{1}, r_{2}\right)$, it follows that $p\left(r_{1}\right)>p\left(r_{2}\right)$. On the other hand, since $\alpha(\lambda)$ is monotone increasing in $\lambda$ (by Lemma 3.1) and $c_{\lambda}(r)$ is monotone increasing in $r$, we have $\alpha\left(c_{\lambda}\left(r_{1}\right)\right)<\alpha\left(c_{\lambda}\left(r_{2}\right)\right)$, which is a contradiction. Hence $p^{\prime}(r) \geq 0$ for $0 \leq r \leq R_{\lambda}$. A similar argument shows that $p^{\prime}(r)$ cannot be identically zero on any intervals, so that $p(r)$ is strictly monotone increasing.

The assertion (2) follows immediately from the above result, because by Lemma 3.1 we have $p(0)=\alpha(\lambda)>0$ and $p\left(R_{\lambda}\right)=\alpha(1)=1$. Differentiating the equation (2.19), we get

$$
\begin{aligned}
\frac{d}{d r}\left(u^{\prime}(r)+\frac{2}{r} u(r)\right) & =-K_{D}^{\prime}\left(c_{\lambda}(r)\right) c_{\lambda}^{\prime}(r)+K_{M}^{\prime}\left(c_{\lambda}(r)\right) c_{\lambda}^{\prime}(r) p(r)+K_{M}\left(c_{\lambda}(r)\right) p^{\prime}(r) \\
& >0 \quad \text { for } 0<r \leq R_{\lambda},
\end{aligned}
$$

because $K_{D}^{\prime}<0, c_{\lambda}^{\prime}>0, K_{M}^{\prime}>0, p>0, K_{M}>0$ and $p^{\prime} \geq 0$, so that (3) holds.

Next we prove assertion (4). Assume that this assertion is false. Since clearly $u(r)$ cannot be identically zero, it follows that two situations may take place: either (a) $\max _{0 \leq r \leq R_{\lambda}} u(r)>0$ or (b) $u(r) \leq 0$ for all $0 \leq r \leq R_{\lambda}, \min _{0 \leq r \leq R_{\lambda}} u(r)<0$, and there exists an $r_{0} \in\left(0, R_{\lambda}\right)$ such that $u\left(r_{0}\right)=0$. In case (a) we let $u\left(\tilde{r}_{0}\right)=$ $\max _{0 \leq r \leq R_{\lambda}} u(r)$. It follows that $u^{\prime}\left(\tilde{r}_{0}\right)=0$ and $u\left(\tilde{r}_{0}\right)>0$, so that

$$
u^{\prime}\left(\tilde{r}_{0}\right)+\frac{2}{\tilde{r}_{0}} u\left(\tilde{r}_{0}\right)>0
$$

On the other hand, denoting by $\left(r_{1}, r_{2}\right)$ the maximal interval containing $\tilde{r}_{0}$ such that $u(r)>0$ for all $r \in\left(r_{1}, r_{2}\right)$, we have $u\left(r_{2}\right)=0$ and $u^{\prime}\left(r_{2}\right) \leq 0$. It follows that

$$
u^{\prime}\left(r_{2}\right)+\frac{2}{r_{2}} u\left(r_{2}\right) \leq 0
$$

This contradicts assertion (3). Similarly, in case (b), by comparing the values of $u^{\prime}(r)+(2 / r) u(r)$ at the points $r_{0}, r_{2}$, where $r_{2} \in\left(r_{0}, R_{\lambda}\right)$ is such that $u\left(r_{2}\right)=$ $\min _{r_{0} \leq r \leq R_{\lambda}} u(r)$, we again get a contradiction. Hence assertion (4) follows.

We now prove that $p^{\prime}(r)>0$ for $0<r<R_{\lambda}$. Indeed, since $p^{\prime}(r) \geq 0$ and $u(r)<0$, we have $u(r) p^{\prime}(r) \leq 0$, so that, by equation $(2.21)$,

$$
K_{P}\left(c_{\lambda}(r)\right)+\left(K_{M}\left(c_{\lambda}(r)\right)-K_{N}\left(c_{\lambda}(r)\right)\right) p(r)-K_{M}\left(c_{\lambda}(r)\right) p^{2}(r) \leq 0
$$

for $0 \leq r \leq R_{\lambda}$. It follows that

$$
p(r) \geq \alpha\left(c_{\lambda}(r)\right) \quad\left(0 \leq r \leq R_{\lambda}\right) .
$$

This further implies that if $p\left(r_{0}\right)=\alpha\left(c_{\lambda}\left(r_{0}\right)\right)$ for some $r_{0} \in\left(0, R_{\lambda}\right)$, then $p^{\prime}\left(r_{0}\right)=$ $\alpha^{\prime}\left(c_{\lambda}\left(r_{0}\right)\right) c_{\lambda}^{\prime}\left(r_{0}\right)>0$. It is now easy to show that for any $r \in\left(0, R_{\lambda}\right), p^{\prime}(r)$ cannot 
be zero. Indeed, if $p^{\prime}(r)=0$ at some point $r$ in $\left(0, R_{\lambda}\right)$, then $p(r)=\alpha\left(c_{\lambda}(r)\right)$, by equation (2.21), so that $p^{\prime}(r)>0$ by the preceding observation, which is a contradiction. Hence $p^{\prime}(r)>0$ for all $0<r<R_{\lambda}$.

Finally, we prove assertion (5). Since $u(r)<0$ for $0<r<R_{\lambda}$ and $u\left(R_{\lambda}\right)=0$, we see that $u^{\prime}\left(R_{\lambda}\right) \geq 0$. Assume that $u^{\prime}\left(R_{\lambda}\right)=0$. Then $u^{\prime}\left(R_{\lambda}\right)+\left(2 / R_{\lambda}\right) u\left(R_{\lambda}\right)=0$, so that, by assertion (3),

$$
u^{\prime}(r)+\frac{2}{r} u(r)<0
$$

for all $0<r<R_{\lambda}$. Multiplying both sides by $r^{2}$ and integrating over $\left[r, R_{\lambda}\right]$ yields $r^{2} u(r)>0$ for all $r \in\left(0, R_{\lambda}\right)$, which is a contradiction to (4). Hence $u^{\prime}\left(R_{\lambda}\right)>0$. Similarly we can prove that $u^{\prime}(0)<0$.

Remark 7.1. (1) The conditions $p \in C^{1}[0, R], u \in C^{1}[0, R]$ imposed in Theorem 7.1 can be replaced with the weaker conditions $p \in C[0, R] \cap C^{1}(0, R), u \in C[0, R] \cap$ $C^{1}(0, R)$. Actually, these conditions can be weakened even further. See Corollary 8.2 in the next section.

(2) From the above proof we see that the inequality (7.1) is strict for $0<r<R_{\lambda}$.

If $\lambda \in\left[\lambda_{\infty}, 1\right)$, then the solution of the initial value problem (2.19)-(2.21) satisfies $u^{\prime}(0)=\beta(\lambda) \geq 0$, and thus, by Theorem 7.1, it cannot be a solution of the free boundary value problem (2.19)-(2.22). Therefore, in the sequel we shall only consider solutions $\left(p_{\lambda \psi}, u_{\lambda \psi}\right)$ for $\lambda \in\left(0, \lambda_{\infty}\right)$.

We write for brevity

$$
p_{\lambda \psi}(r)=p(r), \quad u_{\lambda \psi}(r)=u(r) \quad(0<r \leq \delta) .
$$

Since $p(0)=\alpha(\lambda) \in(0,1), u(0)=0$ and $u^{\prime}(0)=\beta(\lambda)<0$, we can extend (in a unique way) the solution to a maximal interval $0 \leq r \leq R$ such that

$$
0<p(r)<1, \quad u(r)<0
$$

for all $0<r<R$, and either $R=R_{\lambda}$, or $R<R_{\lambda}$ and one of the following three cases occurs:

(i) $u(R)=0 ; \quad$ (ii) $p(R)=1 ; \quad$ (iii) $p(R)=0$.

We shall call $(p, u)$ together with the maximal interval $[0, R]$ a semi-entire solution of the initial value problem (2.19)-(2.21). The following result extends Theorem 7.1 to semi-entire solutions of the initial value problem.

Theorem 7.2. Let $\lambda \in\left(0, \lambda_{\infty}\right)$, and let $(p, u, R)$ be a semi-entire solution of the problem (2.19)-(2.21). Then one of the following four situations occurs:

(a) $R \leq R_{\lambda}$ and

$$
\begin{gathered}
\alpha\left(c_{\lambda}(r)\right)<p(r)<\alpha\left(c_{\lambda}(R)\right), \quad p^{\prime}(r)>0 \text { for } 0<r<R, \\
u(r)<0 \text { for } 0<r<R, \\
u(R)=0
\end{gathered}
$$

furthermore, $p(R)=\alpha\left(c_{\lambda}(R)\right)<1$ if $R<R_{\lambda}$, and $p(R)=1$ if $R=R_{\lambda}$.

(b) $R \leq R_{\lambda}$ and

$$
\begin{gathered}
\alpha\left(c_{\lambda}(r)\right)<p(r)<1, \quad p^{\prime}(r)>0 \text { for } 0<r<R \\
u(r)<0 \text { for } 0<r \leq R \\
p(R)=1
\end{gathered}
$$


(c) $R \leq R_{\lambda}$ and there exists $r_{0} \in(0, R)$ such that

$$
\begin{gathered}
\alpha\left(c_{\lambda}(r)\right)<p(r)<\alpha\left(c_{\lambda}\left(r_{0}\right)\right) \text { for } 0<r<r_{0}, \\
0<p(r)<\alpha\left(c_{\lambda}\left(r_{0}\right)\right) \text { for } r_{0}<r<R, \\
p^{\prime}(r)\left\{\begin{array}{l}
>0 \text { for } 0 \leq r<r_{0} \\
=0 \text { for } r=r_{0}, \\
<0 \text { for } r_{0}<r \leq R, \\
u(r)<\text { for } 0<r \leq R
\end{array}\right.
\end{gathered}
$$

furthermore, either $R<R_{\lambda}, p(R)=0$ or $R=R_{\lambda}, 0 \leq p(R)<1$.

(d) $R \leq R_{\lambda}$ and

$$
\begin{gathered}
0<p(r)<\alpha(\lambda), \quad p^{\prime}(r)<0 \text { for } 0<r<R, \\
u(r)<0 \text { for } 0<r \leq R ;
\end{gathered}
$$

furthermore, either $R<R_{\lambda}, p(R)=0$ or $R=R_{\lambda}, 0 \leq p(R)<1$.

Proof. If $u(R)=0$, then similar arguments as in the proof of Theorem 7.1 show that Case $(a)$ holds. It remains to consider the case $u(R)<0$. If $p^{\prime}(r) \geq 0$ for all $0<r<R$, then Case $(b)$ follows as in Case $(a)$. Suppose next that $p^{\prime}\left(r_{0}\right)<0$ for some $r_{0} \in(0, R)$. As before, we denote by $\left(r_{1}, r_{2}\right)$ the maximal interval in $[0, R]$ containing $r_{0}$ such that $p^{\prime}(r)<0$ for all $r_{1}<r<r_{2}$. We claim that $r_{2}=R$. Indeed, if $r_{2}<R$, then $p^{\prime}\left(r_{2}\right)=0$, so that by $(2.21)$,

$$
p\left(r_{2}\right)=\alpha\left(c_{\lambda}\left(r_{2}\right)\right) .
$$

Since we also have $u\left(r_{1}\right) p^{\prime}\left(r_{1}\right)=0$, no matter whether $r_{1}>0$ or $r_{1}=0$, it follows that

$$
p\left(r_{1}\right)=\alpha\left(c_{\lambda}\left(r_{1}\right)\right) .
$$

However, (7.2) and (7.3) contradict the fact that $p^{\prime}(r)<0$ for $r_{1}<r<r_{2}$. Hence $r_{2}=R$. But,then if $r_{1}=0$, then Case $(d)$ holds, whereas if $r_{1}>0$, then, by a similar argument as before, $p^{\prime}(r)>0$ for all $0<r<r_{1}$, and Case $(c)$ holds.

Corollary 7.3. In cases (a) and (b) of Theorem 7.2, we have

$$
\frac{d}{d r}\left(u^{\prime}(r)+\frac{2}{r} u(r)\right)>0 \text { for } 0<r<R .
$$

Furthermore, in case (a) we also have that $u^{\prime}(R)>0$.

The proof is immediate.

Remark 7.2. From (2.19) we notice that, in case $(a)$,

$$
u^{\prime}(R)=-K_{D}\left(c_{\lambda}(R)\right)+K_{M}\left(c_{\lambda}(R)\right) \alpha\left(c_{\lambda}(R)\right),
$$

so that $\alpha\left(c_{\lambda}(R)\right)>K_{D}\left(c_{\lambda}(R)\right) / K_{M}\left(c_{\lambda}(R)\right)$. This gives a lower estimate for $R$.

A semi-entire solution $(p, u, R)$ with $R<R_{\lambda}$ can be uniquely extended to a larger interval $[0, R+\delta]$ for some $\delta>0$. To see this we consider the system of equations (2.19), (2.21) on the interval $[R, R+\delta]$, with initial values $p(R)$ and $u(R)$. Suppose first that $(p, u, R)$ is as in case $(a)$ of Theorem 7.2. Then $p(R)=\alpha\left(c_{0}\right)\left(c_{0} \equiv c_{\lambda}(R)\right)$, $u(R)=0$, and $u^{\prime}(R)>0$ (by Corollary 7.3). Furthermore, as will be shown in $\S 8$ (see (8.10)), $p^{\prime}(R)$ exists and, in fact,

$$
p^{\prime}(R)=\frac{\left\{K_{P}^{\prime}\left(c_{0}\right)+\left(K_{M}^{\prime}\left(c_{0}\right)-K_{N}^{\prime}\left(c_{0}\right)\right) \alpha\left(c_{0}\right)-K_{M}^{\prime}\left(c_{0}\right) \alpha^{2}\left(c_{0}\right)\right\} c_{\lambda}^{\prime}(R)}{u^{\prime}(R)+2 K_{M}\left(c_{0}\right) \alpha\left(c_{0}\right)-\left(K_{M}\left(c_{0}\right)-K_{N}\left(c_{0}\right)\right)} .
$$


Hence we can make the transformation

$$
p(r)=\alpha\left(c_{0}\right)+(r-R)\left(p^{\prime}(R)+P(r)\right), \quad u(r)=(r-R)\left(u^{\prime}(R)+U(r)\right)
$$

with $P, U$ satisfying $P(R)=0$ and $U(R)=0$. We then get a system of differential equations which is, by the fact $u^{\prime}(R)>0$, similar to that considered in Lemma 5.1 (1) and Theorem 5.3 (1). By similar arguments as before we can then extend the solution uniquely with $u(r)>0$ to $R<r \leq R+\delta$. Notice that, by (7.4) and the last two equalities of (4.10), we clearly have $p^{\prime}(R)>0$, so that if $\delta$ is small enough, then $p^{\prime}(r)>0$ for $R<r \leq R+\delta$, which further implies that $\alpha\left(c_{0}\right)<p(r)<1$ for $R<r \leq R+\delta$.

Suppose next that $(p, u, R)$ is as in one of the cases $(b)-(d)$. Then, since $u(R) \neq 0$, the system of equations (2.19) and (2.21) is a regular system at the point $r=R$. We can therefore apply the classical theory of ODEs to uniquely extend the solution to a larger interval $[0, R+\delta]$, although the condition $0 \leq p(r) \leq 1$ may no longer be satisfied for $R<r \leq R+\delta$.

The following theorem asserts that the semi-entire solution can be further extended to the entire interval $0 \leq r \leq R_{\lambda}$, unless it blows up at some point $\bar{R} \in\left(R+\delta, R_{\lambda}\right]$.

Theorem 7.4. Let $\lambda \in\left(0, \lambda_{\infty}\right)$ and let $(p, u, R)$ be a semi-entire solution of the system (2.19)-(2.21). Assume that $R<R_{\lambda}$. Then we have the following conclusions:

(1) If $(p, u, R)$ is as in case (a) of Theorem 7.2, then $(p(r), u(r))$ can be extended to the entire interval $\left[0, R_{\lambda}\right]$, satisfying

$$
\alpha\left(c_{\lambda}(R)\right)<p(r)<\alpha\left(c_{\lambda}(r)\right), \quad p^{\prime}(r)>0 \quad \text { and } u(r)>0
$$

for $R<r \leq R_{\lambda}$.

(2) If $(p, u, R)$ is as in case $(b)$, then $(p(r), u(r))$ can be extended either to the entire interval $\left[0, R_{\lambda}\right]$, satisfying

$$
p(r)>1, \quad p^{\prime}(r)>0 \quad \text { and } u(r)<0
$$

for $R<r \leq R_{\lambda}$, or to a half-open interval $[0, \bar{R})\left(R<\bar{R} \leq R_{\lambda}\right)$ such that (7.6) holds for $R<r<\bar{R}$, and

$$
\lim _{r \rightarrow \bar{R}} p(r)=+\infty, \quad \lim _{r \rightarrow \bar{R}} u(r)=0 .
$$

(3) If $(p, u, R)$ is as in cases $(c)$ and $(d)$, then $(p(r), u(r))$ can be extended to the entire interval $\left[0, R_{\lambda}\right]$, satisfying

$$
\min _{R \leq r \leq R_{\lambda}} \alpha_{-}\left(c_{\lambda}(r)\right) \leq p(r)<0 \text { and } u(r)<0
$$

for $R<r \leq R_{\lambda}$, where $\alpha_{-}$is the negative root of the equation (3.1), i.e.,

$$
\alpha_{-}(\lambda)=\frac{1}{2 K_{M}(\lambda)}\left(K_{M}(\lambda)-K_{N}(\lambda)-\sqrt{\left(K_{M}(\lambda)-K_{N}(\lambda)\right)^{2}+4 K_{M}(\lambda) K_{P}(\lambda)}\right) .
$$

Proof. Consider Case (1) first. Let $\left(R, r_{0}\right)$ be the largest open interval contained in $\left(R, R_{\lambda}\right)$ such that the solution exists and $p^{\prime}(r)>0$ and $u(r)>0$ for all $r \in\left(R, r_{0}\right)$. We claim that $r_{0}=R_{\lambda}$ and $(p(r), u(r))$ is well-defined at $r=R_{\lambda}$. Indeed, by (2.21) and (2.19), the conditions $p^{\prime}(r)>0$ and $u(r)>0$ imply that

$$
\alpha\left(c_{\lambda}(R)<p(r)<\alpha\left(c_{\lambda}(r)\right), \quad\left|u^{\prime}(r)+\frac{2}{r} u(r)\right| \leq \text { const. }<\infty\right.
$$


for $R<r<r_{0}$, so that $p\left(r_{0}\right)=\lim _{r \rightarrow r_{0}-0} p(r)$ and $u\left(r_{0}\right)=\lim _{r \rightarrow r_{0}-0} u(r)$ exist. Furthermore, since $p^{\prime}(r)>0$, by (2.19) we see that $u^{\prime}(r)+(2 / r) u(r)$ is monotone increasing in $\left(R, r_{0}\right)$, which implies that $\left(r^{2} u(r)\right)^{\prime}>r^{2}\left(u^{\prime}(R)+(2 / R) u(R)\right)>0$ and, therefore, $u\left(r_{0}\right)>0$. It follows that the system of equations (2.19), (2.21) is regular at $r_{0}$, so that $p^{\prime}\left(r_{0}\right)$ exists. We claim that $p^{\prime}\left(r_{0}\right)>0$. Indeed, if $p^{\prime}\left(r_{0}\right)=0$, then $p\left(r_{0}\right)=\alpha\left(c_{\lambda}\left(r_{0}\right)\right)$. But then, since $p(r)<\alpha\left(c_{\lambda}(r)\right)$ for $R<r<r_{0}$, we infer that $p^{\prime}\left(r_{0}\right) \geq \alpha^{\prime}\left(c_{\lambda}\left(r_{0}\right)\right) c_{\lambda}^{\prime}\left(r_{0}\right)>0$, which is a contradiction. Hence $p^{\prime}\left(r_{0}\right)>0$. It follows that if $r_{0}<R_{\lambda}$, then we can extend $(p, u)$ to a larger interval on which the conditions $p^{\prime}(r)>0$ and $u(r)>0$ still hold, which is contrary to the maximality of $r_{0}$. Hence $r_{0}=R_{\lambda}$, and Case (1) follows.

Consider Case (2). Since $p(R)=1$ and $u(R)<0$, it is easy to see (by $(2.21)$ ) that $p^{\prime}(R)>0$, so that (7.6) holds for $r$ larger than but near $R$. Let $\left(R, r_{0}\right)$ be the largest open interval such that the solution exists and $p^{\prime}(r)>0, u(r)<0$ for all $R<r<r_{0}$. Then either $p\left(r_{0}\right)=\lim _{r \rightarrow r_{0}-0} p(r)$ exists and is greater than 1 , or $\lim _{r \rightarrow r_{0}-0} p(r)=+\infty$. In the first case it follows from (2.19) that $u\left(r_{0}\right)=\lim _{r \rightarrow r_{0}-0} u(r)$ also exists. Since $p^{\prime}(r)>0$ for $R<r<r_{0}$, we have

$$
p\left(r_{0}\right)>p(R)=1 \geq \alpha\left(c_{\lambda}\left(r_{0}\right)\right),
$$

so that the right-hand side of $(2.21)$ is $\neq 0$; hence $u\left(r_{0}\right) \neq 0$ and, therefore, $u\left(r_{0}\right)<0$ and $p^{\prime}\left(r_{0}\right)>0$. But then $r_{0}=R_{\lambda}$, for otherwise we can extend $(p, u)$ to a larger interval such that (7.6) still holds, which contradicts the maximality of $\left(r, r_{0}\right)$. Consider next the second case, where $p\left(r_{0}-0\right)=+\infty$. We claim that

$$
\lim _{r \rightarrow r_{0}} u(r)=0 \text {. }
$$

Indeed, since $u(r)<0$ for $r<r_{0}$, by (2.19) we have

$$
u^{\prime}(r)>-K_{D}\left(c_{\lambda}(r)\right)+K_{M}\left(c_{\lambda}(r)\right) p(r) \rightarrow+\infty
$$

as $r \rightarrow r_{0}$, so that $u(r)$ is monotone increasing for $r$ near $r_{0}$. It follows that $u(r)$ has a limit as $r$ approaches $r_{0}$. We claim that $\kappa \equiv \lim _{r \rightarrow r_{0}} u(r)$ is equal to zero. Indeed, suppose $\kappa<0$ and let

$$
y(r)=\left\{\begin{array}{l}
\frac{1}{p(r)} \text { for } 0<r<r_{0} \\
0 \text { for } r=r_{0} .
\end{array}\right.
$$

Clearly, $y \in C\left(0, r_{0}\right] \cap C^{1}\left(0, r_{0}\right)$, and by (2.21),

$$
\begin{aligned}
\lim _{r \rightarrow r_{0}} y^{\prime}(r) & =-\lim _{r \rightarrow r_{0}} \frac{K_{P}\left(c_{\lambda}(r)\right)+\left(K_{M}\left(c_{\lambda}(r)\right)-K_{N}\left(c_{\lambda}(r)\right)\right) p(r)-K_{M}\left(c_{\lambda}(r)\right) p^{2}(r)}{u(r) p^{2}(r)} \\
& =\frac{K_{M}\left(c_{\lambda}\left(r_{0}\right)\right)}{\kappa} .
\end{aligned}
$$

Hence

$$
y(r)=-\frac{K_{M}\left(c_{\lambda}\left(r_{0}\right)\right)}{\kappa}\left(r_{0}-r\right)+o\left(r_{0}-r\right) \quad \text { as } r \rightarrow r_{0},
$$

so that

$$
p(r)=-\frac{\kappa}{K_{M}\left(c_{\lambda}(r)\right)} \cdot \frac{1}{r_{0}-r}(1+o(1)) \quad \text { as } r \rightarrow r_{0} .
$$

By (2.19), we then get

$$
u^{\prime}(r)=-\frac{\kappa}{r_{0}-r}(1+o(1)) \quad \text { as } r \rightarrow r_{0},
$$


so that $u(r) \rightarrow-\infty$ as $r \rightarrow r_{0}$, which is a contradiction. This proves (7.10). Thus assertion (2) holds with $\bar{R}=r_{0}$.

Finally, we consider case (3). Since $p(R)=0$ and $u(R)<0$, by (2.21) it follows that $p^{\prime}(R)<0$, so that $\alpha_{-}(r)<p(r)<0, p^{\prime}(r)<0$ and $u(r)<0$ for $r$ larger than but near $R$. Let $\left(R, r_{0}\right)$ be the largest open interval such that the solution exists and

$$
p(r)<0, \quad u(r)<0
$$

for $R<r<r_{0}$. From (2.21) we infer that

$$
p^{\prime}(r)\left\{\begin{aligned}
<0 & \text { if } \alpha_{-}\left(c_{\lambda}(r)\right)<p(r)<0 \\
>0 & \text { if } p(r)<\alpha_{-}\left(c_{\lambda}(r)\right)
\end{aligned}\right.
$$

for all $R<r<r_{0}$, and this implies that

$$
p(r) \geq \min _{R \leq r \leq r_{0}} \alpha_{-}\left(c_{\lambda}(r)\right) \geq \min _{R \leq r \leq R_{\lambda}} \alpha_{-}\left(c_{\lambda}(r)\right)
$$

for $R<r<r_{0}$, so that $p(r)$ is bounded. Using (2.19), we find that $u\left(r_{0}\right) \equiv$ $\lim _{r \rightarrow r_{0}} u(r)$ exists and is negative and $u(r)$ has a negative upper bound in the interval $R<r<r_{0}$. Hence, by (2.21), $p^{\prime}(r)$ is bounded for $R<r<r_{0}$, which implies that also $p\left(r_{0}\right) \equiv \lim _{r \rightarrow r_{0}} p(r)$ exists. We claim that $p\left(r_{0}\right)<0$. Indeed, if $p\left(r_{0}\right)=0$, then on the one hand, since $p(r)<0$ for $R<r<r_{0}, p^{\prime}\left(r_{0}\right) \geq 0$; but on the other hand, it follows from $(2.21)$ and the fact $u\left(r_{0}\right)<0$ that $p^{\prime}\left(r_{0}\right)<0$, which is a contradiction. Recalling the maximality of $\left(R, r_{0}\right)$, we conclude that $r_{0}=R_{\lambda}$, and (7.8) holds for all $R<r \leq R_{\lambda}$.

Remark 7.3. Every entire solution is analytic at all points except possibly at $r=0$. Indeed, at a point where $u$ does not vanish, the analyticity of $p$ and $u$ follows immediately from classical results, and where $u$ vanishes, the analyticity follows as in the proof of Theorem 4.1, noting that if $u\left(r_{0}\right)=0$ and $r_{0}>0$, then $u^{\prime}\left(r_{0}\right)>0$ so that in the equations analogous to (4.9) the coefficients of $p^{(k)}\left(r_{0}\right)$ are uniformly positive.

Remark 7.4. We can prove that if $(p, u)$ is as in (7.7), then there exists a constant $\kappa>0$ such that

$$
p(r)=\frac{\kappa}{\sqrt{\overline{R-r}}}(1+o(1)), \quad u(r)=-2 K_{M}\left(c_{\lambda}(\bar{R})\right) \kappa \sqrt{\bar{R}-r}(1+o(1))
$$

as $r \rightarrow \bar{R}$. Since we shall not use this result, we omit the proof.

Definition 7.1. The solutions obtained in Theorem 7.4 are called entire solutions. We distinguish four types of such solutions in accordance with Theorem 7.4:

(i) If $0<p(r) \leq 1, p^{\prime}(r)>0$ for $0<r \leq R_{\lambda}$, and there exists an $R \in\left(0, R_{\lambda}\right]$ such that $u(r)<0$ for $0<r<R, u(R)=0$, and $u(r)>0$ for $R<r \leq R_{\lambda}$, then we call the solution of the initial value problem (2.19)-(2.21) a subsolution of the free boundary problem (2.19)-(2.22); in particular, if $R<R_{\lambda}$, then we call it a strict subsolution.

(ii) If $0<p(r)<1, p^{\prime}(r)>0$ and $u(r)<0$ for $0<r<R_{\lambda}$ and $p\left(R_{\lambda}\right)=1$, $u\left(R_{\lambda}\right)<0\left(\Longrightarrow p^{\prime}\left(R_{\lambda}\right)=0\right)$, then we call it a supersolution of the free boundary problem (2.19) $-(2.22)$.

(iii) If $p^{\prime}(r)>0, u(r)<0$ for $0<r \leq R_{\lambda}$, and there exists $R \in\left(0, R_{\lambda}\right]$ such that $0<p(r)<1$ for $0<r<R, p(R)=1$, and $p(r)>1$ for $R<r \leq R_{\lambda}$, then we call it a bounded upper solution, whereas if there exists $\bar{R} \in\left(0, R_{\lambda}\right]$ such that $(p, u)$ is 
only defined for $0<r<\bar{R}, p^{\prime}(r)>0, u(r)<0$ for $0<r<\bar{R}$, and (7.7) holds, then we call it an unbounded upper solution.

(iv) If the solution is as in Theorem $7.4(3)$, or equivalently, $p^{\prime}\left(r_{0}\right)<0$ for some $r_{0} \in\left(0, R_{\lambda}\right)$, then we call it a lower solution.

Note that, by the definition, a supersolution is also a bounded upper solution, and a solution of the free boundary problem $(2.19)-(2.22)$ is a subsolution.

In $\S \S 9,10$ we shall prove that, for each $\lambda \in\left(0, \lambda_{\infty}\right)$, there exists a unique $\bar{\psi}=$ $\bar{\psi}(\lambda) \in \mathbf{R}$ such that $\left(p_{\lambda \psi}, u_{\lambda \psi}\right)$ is an upper solution for $\psi>\bar{\psi}$ and a lower solution for $\psi<\bar{\psi}$, whereas $\left(p_{\lambda}, u_{\lambda}\right) \equiv\left(p_{\lambda \bar{\psi}}, u_{\lambda \bar{\psi}}\right)$ is either a subsolution or a supersolution. Moreover, if $\left(p_{\lambda_{1}}, u_{\lambda_{1}}\right)$ is a subsolution and $\left(p_{\lambda_{2}}, u_{\lambda_{2}}\right)$ is a supersolution, then there exists a $\lambda^{*}$ between $\lambda_{1}$ and $\lambda_{2}$ such that $\left(p_{\lambda^{*}}, u_{\lambda^{*}}\right)$ is a solution of the free boundary problem (2.19)-(2.22).

\section{WEAK SOLUTIONS OF THE INITIAL VALUE PROBLEM}

In this section we reformulate the problem (2.19)-(2.21) as a system of integral equations, and use it to introduce the concept of weak solutions. The integral equation formulation presented here will enable us to work with weak limits of solutions.

Theorem 8.1. Suppose that $p(r), u(r) \in L^{\infty}[0, R] \cap C^{1}(0, R]$ and they satisfy equations (2.19), (2.21) for $0<r \leq R$, where $0<R \leq R_{\lambda}$, and $R_{\lambda}$ is as in (2.18). Then $p(r), u(r)$ also satisfy the equations

$$
\begin{gathered}
u(r)=\frac{1}{r^{2}} \int_{0}^{r}\left(-K_{D}\left(c_{\lambda}(\rho)\right)+K_{M}\left(c_{\lambda}(\rho)\right) p(\rho)\right) \rho^{2} d \rho \\
u(r) p(r)=\frac{1}{r^{2}} \int_{0}^{r}\left(K_{P}\left(c_{\lambda}(\rho)\right)+\left(K_{B}\left(c_{\lambda}(\rho)\right)-K_{N}\left(c_{\lambda}(\rho)\right)\right) p(\rho)\right) \rho^{2} d \rho
\end{gathered}
$$

for $0<r \leq R$. Conversely, if $p(r), u(r)$ belong to $L^{\infty}[0, R]$ and satisfy the system of equations (8.1), (8.2) for almost all $0<r \leq R$, and

$$
p(r) \geq 0 \text { for } 0 \leq r \leq R,
$$

then, after modifying the values of $u$ and $p$ on a subset of measure zero, $p \in$ $C[0, R] \cap C^{1}(0, R], u \in C^{1}[0, R]$, and they satisfy $(2.19)-(2.21)$ for all $0<r \leq R$; furthermore,

(i) $p(0)$ satisfies the equation

$$
K_{P}(\lambda)+\left(K_{M}(\lambda)-K_{N}(\lambda)\right) p(0)-K_{M}(\lambda) p^{2}(0)=0
$$

(ii) if $\gamma_{1}(\lambda)>0$, then $p(r)$ is continuously differentiable at $r=0$, and $p^{\prime}(0)=0$; if $\gamma_{1}(\lambda)=0$, then $p(r)$ is continuously differentiable at $r=0$, but $p^{\prime}(0)$ can be any real number; if $\gamma_{1}(\lambda)<0(\Longleftrightarrow-1<\sigma(\lambda)<0)$, then the limit

$$
\omega=\lim _{r \rightarrow 0} p^{\prime}(r) r^{-\sigma(\lambda)}
$$

exists, and if $\omega=0$, then $p(r)$ is continuously differentiable at $r=0$ and $p^{\prime}(0)=0$.

Proof. Suppose first that $p(r), u(r)$ satisfy (2.19), (2.21). Mutiplying (2.19) with $r^{2}$ and integrating over $[0, r]$ (for arbitrary $0<r \leq R$ ) immediately yields (8.1). Next, multiplying (2.21) with $r^{2}$, integrating over $[0, r]$, and using integration by parts and equation (2.19), we obtain (8.2). 
Now suppose that $p, u \in L^{\infty}[0, R]$ and they satisfy (8.1)-(8.3) for almost all $0<r \leq R$. We modify the values of $u(r)$ on a subset of measure zero by replacing it with the right-hand side of (8.1). Then $u(r)$ is continuous for all $0 \leq r \leq R$, and $u(0)=0$. Similarly, after modifying the values of $p(r)$ on a subset of measure zero of the open set

$$
O=\{r \in(0, R): u(r) \neq 0\},
$$

we may assume that $p(r)$ satisfies (8.2) and is continuous at all $r \in O$. From (8.1), (8.2) we further conclude that $u(r), p(r)$ are continuously differentiable in $O$. Let

$$
\Sigma=\{r \in[0, R]: u(r)=0\} .
$$

We claim that

$$
\operatorname{meas}(\Sigma)=0
$$

Indeed, if meas $(\Sigma)>0$, then meas $\left(\Sigma^{*}\right)=\operatorname{meas}(\Sigma)>0$, where $\Sigma^{*}$ is the set of Lebesgue points of $\Sigma$. Since at each point of $\Sigma$ we have

$$
\begin{gathered}
\left.\int_{0}^{r}\left(-K_{D}\left(c_{\lambda}(\rho)\right)+K_{M}\left(c_{\lambda}(\rho)\right) p(\rho)\right) \rho^{2} d \rho=0 \quad \text { (by }(8.1)\right), \\
\left.\int_{0}^{r}\left(K_{P}\left(c_{\lambda}(\rho)\right)+\left(K_{B}\left(c_{\lambda}(\rho)\right)-K_{N}\left(c_{\lambda}(\rho)\right)\right) p(\rho)\right) \rho^{2} d \rho=0 \quad \text { (by } \quad(8.2)\right),
\end{gathered}
$$

it follows that, for almost all $r \in \Sigma^{*}$,

$$
\begin{gathered}
-K_{D}\left(c_{\lambda}(r)\right)+K_{M}\left(c_{\lambda}(r)\right) p(r)=0, \\
K_{P}\left(c_{\lambda}(r)\right)+\left(K_{B}\left(c_{\lambda}(r)\right)-K_{N}\left(c_{\lambda}(r)\right)\right) p(r)=0 .
\end{gathered}
$$

Eliminating $p(r)$, we get

$$
\varphi(r) \equiv K_{M}\left(c_{\lambda}(r)\right) K_{P}\left(c_{\lambda}(r)\right)+\left(K_{B}\left(c_{\lambda}(r)\right)-K_{N}\left(c_{\lambda}(r)\right)\right) K_{D}\left(c_{\lambda}(r)\right)=0
$$

a.e. in $\Sigma^{*}$. On the other hand, if we denote by $c^{*}$ the unique positive number such that $K_{B}\left(c^{*}\right)=K_{Q}\left(c^{*}\right)$, then by writing $\varphi=K_{B} K_{P}+\left(K_{B}-K_{Q}\right) K_{D}$, we easily verify that $\varphi^{\prime}(r)>0$ if $c_{\lambda}(r) \leq c^{*}$ and $\varphi(r)>0$ if $c_{\lambda}(r) \geq c^{*}$, so that $\varphi(r)$ has at most one zero, contradicting the fact that $\varphi=0$ on a set of positive measure. This completes the proof of (8.7). Since meas $(\Sigma)=0$, we may redefine $p(r)$ for $r \in \Sigma$ by

$$
p(r)=\alpha\left(c_{\lambda}(r)\right) \quad(r \in \Sigma) ;
$$

this does not change the fact that both (8.1) and (8.2) are satisfied for all $0<r \leq R$. With the definition (8.8) at hand, we shall be able to prove that $p(r)$ is continuous for $r \in \Sigma$.

Differentiating (8.1) and (8.2), one easily finds that $u(r), p(r)$ satisfy equations (2.19) and (2.21) in $O$. Clearly, $O$ consists of a countable number of disjoint open intervals. Let $\left(r_{1}, r_{2}\right)$ be any one of these open intervals (so that $u\left(r_{1}\right)=0$, and $u\left(r_{2}\right)=0$ if $\left.r_{2}<R\right)$. Arguing similarly as in the proof of Theorem 7.2, we infer that $p^{\prime}(r)$ changes sign at most once in $\left(r_{1}, r_{2}\right)$. It follows that $p(r)$ has limits as $r \rightarrow r_{1}+0$ and $r \rightarrow r_{2}-0$. By (8.1), (8.2) we have

$$
p(r)=\frac{\int_{0}^{r}\left(K_{P}\left(c_{\lambda}(\rho)\right)+\left(K_{B}\left(c_{\lambda}(\rho)\right)-K_{N}\left(c_{\lambda}(\rho)\right)\right) p(\rho)\right) \rho^{2} d \rho}{\int_{0}^{r}\left(-K_{D}\left(c_{\lambda}(\rho)\right)+K_{M}\left(c_{\lambda}(\rho)\right) p(\rho)\right) \rho^{2} d \rho}
$$


for $r_{1}<r<r_{2}$. Using l'Hospital's rule, we deduce that

(8.9)

$$
K_{P}\left(c_{\lambda}\left(r_{1}\right)\right)+\left(K_{M}\left(c_{\lambda}\left(r_{1}\right)\right)-K_{N}\left(c_{\lambda}\left(r_{1}\right)\right)\right) p\left(r_{1}+0\right)-K_{M}\left(c_{\lambda}\left(r_{1}\right)\right) p^{2}\left(r_{1}+0\right)=0 .
$$

By Lemma 3.1 and (8.8), it follows that

$$
p\left(r_{1}+0\right)=\alpha\left(c_{\lambda}\left(r_{1}\right)\right)=p\left(r_{1}\right) .
$$

As for $p\left(r_{2}-0\right)$, if either $r_{2}<R\left(\Longrightarrow u\left(r_{2}\right)=0\right)$ or $r_{2}=R$ and $u(R)=0$, then a similar argument as above shows that $p\left(r_{2}-0\right)=p\left(r_{2}\right)=\alpha\left(c_{\lambda}\left(r_{2}\right)\right)$. If instead $r_{2}=R$ and $u(R) \neq 0$, then clearly $p(R-0)=p(R)$. Hence, $p$ is right continuous at $r_{1}$ and left continuous at $r_{2}$. This further implies that $u^{\prime}\left(r_{1}+0\right)$ and $u^{\prime}\left(r_{2}-0\right)$ exist. Using arguments similar to those used in the proof of Theorem 7.1, we can show, if either $r_{2}<R$, or $r_{2}=R$ and $u(R)=0$, that $p^{\prime}(r) \geq 0$ and $u(r)<0$ for $r_{1}<r<r_{2}$, and $u^{\prime}\left(r_{1}+0\right)<0, u^{\prime}\left(r_{2}-0\right)>0$. From the above discussion it follows, in particular, that if $r_{0}$ is an isolated point in $\Sigma$, then $p$ is continuous at $r_{0}$.

Consider next the case where $r_{0}$ is an accumulation point of $\Sigma$. Since $u$ cannot be identically zero at any intervals, $O$ is dense in $[0, R]$ so that $r_{0}$ is an accumulation point of $O$. Let $\left\{r_{n}\right\}_{n=1}^{\infty}$ be a sequence of points in $[0, R]$ converging to $r_{0}$. By splitting it into two distinct subsequences if necessary, we may assume that $\left\{r_{n}\right\}_{n=1}^{\infty}$ is either monotone increasing or monotone decreasing. Consider the case where $\left\{r_{n}\right\}_{n=1}^{\infty}$ is monotone increasing; the monotone decreasing case can be dealt with in a similar way. By further splitting the sequence into two subsequences if necessary, we may assume that $\left\{r_{n}\right\}_{n=1}^{\infty}$ satisfies one of the following two conditions:

(a) $r_{n} \in \Sigma, n=1,2, \cdots$;

(b) $r_{n} \in O, n=1,2, \cdots$.

In Case (a) we have $p\left(r_{n}\right)=\alpha\left(c_{\lambda}\left(r_{n}\right)\right)$, so that

$$
\lim _{n \rightarrow \infty} p\left(r_{n}\right)=\lim _{n \rightarrow \infty} \alpha\left(c_{\lambda}\left(r_{n}\right)\right)=\alpha\left(c_{\lambda}\left(r_{0}\right)\right)=p\left(r_{0}\right) .
$$

In Case (b) two situations may occur:

(b1) There is $\delta>0$ such that $\left(r_{0}-\delta, r_{0}\right) \subset O$;

(b2) $r_{0}$ is the limit of an increasing sequence of points in $\Sigma$.

In the first situation we know by the preceding analysis that $\lim _{r \rightarrow r_{0}-0} p(r)=p\left(r_{0}\right)$, so that

$$
\lim _{n \rightarrow \infty} p\left(r_{n}\right)=p\left(r_{0}\right)
$$

In the second situation we denote by $\left(r_{1 n}, r_{2 n}\right)$ the open interval contained in $O$ such that $r_{1 n}<r_{n}<r_{2 n}$ and $u\left(r_{1 n}\right)=u\left(r_{2 n}\right)=0, n=1,2, \cdots$. Since $p$ is monotone increasing in $\left(r_{1 n}, r_{2 n}\right)$,

$$
\alpha\left(c_{\lambda}\left(r_{1 n}\right)\right)=p\left(r_{1 n}+0\right)<p\left(r_{n}\right)<p\left(r_{2 n}-0\right)=\alpha\left(c_{\lambda}\left(r_{2 n}\right)\right), \quad n=1,2, \cdots,
$$

so that

$$
\lim _{n \rightarrow \infty} p\left(r_{n}\right)=\lim _{n \rightarrow \infty} \alpha\left(c_{\lambda}\left(r_{1 n}\right)\right)=\lim _{n \rightarrow \infty} \alpha\left(c_{\lambda}\left(r_{2 n}\right)\right)=\alpha\left(c_{\lambda}\left(r_{0}\right)\right)=p\left(r_{0}\right) .
$$

Hence $p$ is continuous at $r_{0}$.

Having proved that $p$ is continuous at any point of $\Sigma$, we conclude that $p \in$ $C[0, R]$ and, consequently, $u \in C^{1}[0, R]$. (The assertion that $\lim _{r \rightarrow 0} u^{\prime}(r)$ exists can be easily verified using (8.1) and (2.19).) Furthermore, since $p$ is monotone increasing in every open interval $\left(r_{1}, r_{2}\right)$ of $O$ such that either $r_{2}<R$ or $r_{2}=R$ 
and $u(R)=0$, and since $p$ is also monotone increasing in $\Sigma$, we conclude that if $\Sigma$ contains more than one point, $p$ is monotone increasing in the interval $\left[0, r^{*}\right]$, where

$$
r^{*}=\sup \{r: r \in \Sigma\}
$$

if $r^{*}<R$, then either $p$ is monotone increasing in $\left[r^{*}, R\right]$ or it changes monotonicity once, say, at the point $r^{* *} \in\left(r^{*}, R\right)$, and $p$ is increasing in $\left[0, r^{* *}\right]$ and decreasing in $\left[r^{* *}, R\right]$. It follows that $u^{\prime}(r)+(2 / r) u(r)$ is monotone increasing for either $0<r \leq R$ or $0<r \leq r^{* *}$ (if $r^{* *}<R$ ). This implies, by similar arguments as in the proofs of Theorems 7.1 and 7.2, that either $\Sigma=\{0\}$ or $\Sigma=\left\{0, r_{0}\right\}$ for some $0<r_{0} \leq R$.

We next prove that if $\Sigma=\left\{0, r_{0}\right\}\left(0<r_{0} \leq R\right)$, then

$$
\begin{aligned}
\lim _{r \rightarrow r_{0}-0} p^{\prime}(r) & =\frac{\left\{K_{P}^{\prime}\left(c_{0}\right)+\left(K_{M}^{\prime}\left(c_{0}\right)-K_{N}^{\prime}\left(c_{0}\right)\right) \alpha\left(c_{0}\right)-K_{M}^{\prime}\left(c_{0}\right) \alpha^{2}\left(c_{0}\right)\right\} c_{\lambda}^{\prime}\left(r_{0}\right)}{u^{\prime}\left(r_{0}\right)+2 K_{M}\left(c_{0}\right) \alpha\left(c_{0}\right)-\left(K_{M}\left(c_{0}\right)-K_{N}\left(c_{0}\right)\right)} \\
& \equiv I\left(r_{0}\right),
\end{aligned}
$$

where $c_{0}=c_{\lambda}\left(r_{0}\right)$. (Note that $I\left(r_{0}\right)$ is the derivative of $p$ at $r_{0}$ formally computed by differentiating equation (2.21) at $r=r_{0}$ and setting $u\left(r_{0}\right) p^{\prime \prime}\left(r_{0}\right)=0$.) To this end we differentiate $(2.21)$ for $0<r<r_{0}$ and divide it by $u(r)$ to get

$$
p^{\prime \prime}(r)+\frac{a(r)}{u(r)} p^{\prime}(r)=\frac{b(r)}{u(r)},
$$

where

$$
\begin{aligned}
& a(r)=u^{\prime}(r)+2 K_{M}\left(c_{\lambda}(r)\right) p(r)-\left(K_{M}\left(c_{\lambda}(r)\right)-K_{N}\left(c_{\lambda}(r)\right)\right), \\
& b(r)=\left\{K_{P}^{\prime}\left(c_{\lambda}(r)\right)+\left(K_{M}^{\prime}\left(c_{\lambda}(r)\right)-K_{N}^{\prime}\left(c_{\lambda}(r)\right)\right) p(r)-K_{M}^{\prime}\left(c_{\lambda}(r)\right) p^{2}(r)\right\} c_{\lambda}^{\prime}(r) .
\end{aligned}
$$

Let $\bar{r}$ be an arbitrary point in $\left(0, r_{0}\right)$ and set

$$
A(r)=\int_{\bar{r}}^{r} \frac{a(\rho)}{u(\rho)} d \rho, \quad 0<r<r_{0} .
$$

Since $u(r)=u^{\prime}\left(r_{0}\right)\left(r-r_{0}\right)+o\left(r-r_{0}\right)$ as $r \rightarrow r_{0}$ and $u^{\prime}\left(r_{0}\right)>0, a\left(r_{0}\right)>0$ (by $(3.2))$, it follows that

$$
A(r)=\frac{a\left(r_{0}\right)}{u^{\prime}\left(r_{0}\right)} \log \left|r-r_{0}\right| \cdot(1+o(1)) \quad \text { as } r \rightarrow r_{0}-0
$$

We multiply (8.11) with $e^{A(r)}$ and integrate over $[\bar{r}, r]$ to get

$$
p^{\prime}(r) e^{A(r)}=p^{\prime}(\bar{r}) e^{A(\bar{r})}+\int_{\bar{r}}^{r} \frac{b(\rho)}{u(\rho)} e^{A(\rho)} d \rho .
$$

By (8.12) we have

$$
\frac{b(r)}{u(r)} e^{A(r)}=-\frac{b\left(r_{0}\right)}{u^{\prime}\left(r_{0}\right)}\left|r-r_{0}\right|^{\nu(1+o(1))}(1+o(1)) \quad \text { as } r \rightarrow r_{0}-0,
$$

where

$$
\nu=\frac{a\left(r_{0}\right)}{u^{\prime}\left(r_{0}\right)}-1=\frac{\left.\left.2 K_{M}\left(c_{0}\right) \alpha\left(c_{0}\right)-\left(K_{M}\left(c_{0}\right)\right)-K_{N}\left(c_{0}\right)\right)\right)}{u^{\prime}\left(r_{0}\right)}>0 \quad(\text { by }(3.2)) .
$$

It follows that $(b(r) / u(r)) e^{A(r)}$ is integrable on $\left[\bar{r}, r_{0}\right)$. Hence, by $(8.13)$,

$$
\kappa \equiv \lim _{r \rightarrow r_{0}-0} p^{\prime}(r) e^{A(r)}
$$


exists, and

$$
\kappa=p^{\prime}(\bar{r}) e^{A(\bar{r})}+\int_{\bar{r}}^{r_{0}} \frac{b(\rho)}{u(\rho)} e^{A(\rho)} d \rho .
$$

We claim that $\kappa=0$. To see this we assume the converse: $\kappa \neq 0$. Since $p^{\prime}(r) \geq 0$ for $0<r<r_{0}$, we have $\kappa>0$. From the definition of $\kappa$ and (8.12) it then follows that

$$
\lim _{r \rightarrow r_{0}-0} p^{\prime}(r)=+\infty
$$

We now write, for $0<r<r_{0}$,

$$
p^{\prime}(r)=\frac{K_{P}\left(c_{\lambda}(r)\right)+\left(K_{M}\left(c_{\lambda}(r)-K_{N}\left(c_{\lambda}(r)\right)\right) p(r)-K_{M}\left(c_{\lambda}(r)\right) p^{2}(r)\right.}{u(r)},
$$

and by l'Hospital's rule,

$$
\begin{aligned}
& \lim _{r \rightarrow r_{0}-0} p^{\prime}(r) \\
& =\lim _{r \rightarrow r_{0}-0} \frac{\left(K_{P}^{\prime}+\left(K_{M}^{\prime}-K_{N}^{\prime}\right) p-K_{M}^{\prime} p^{2}\right) c_{\lambda}^{\prime}(r)-\left(2 K_{M} p-\left(K_{M}-K_{N}\right)\right) p^{\prime}(r)}{u^{\prime}(r)} .
\end{aligned}
$$

However, by (8.15), the left-hand side is $+\infty$ while the right-hand side is $-\infty$, which is a contradiction.

Having proved that $\kappa=0$, we infer from (8.13) and (8.14) that

$$
p^{\prime}(r)=-\int_{r}^{r_{0}} \frac{b(\rho)}{u(\rho)} e^{A(\rho)} d \rho / e^{A(r)},
$$

and, invoking l'Hospital's rule again, (8.10) easily follows.

Similarly we can prove that $\lim _{r \rightarrow r_{0}+0} p^{\prime}(r)=I\left(r_{0}\right)$. Hence $p(r)$ is continuously differentiable at $r_{0}$.

Finally, assertion (i) follows immediately from (8.9) by taking $r_{1}=0$, and assertion (ii) follows from (8.13) and

$$
\frac{a(0)}{u^{\prime}(0)}=\frac{\gamma_{1}(\lambda)}{\beta(\lambda)}=-\sigma(\lambda)
$$

by using previous arguments. This completes the proof of Theorem 8.1.

Corollary 8.2. Let $\left(p, u, R_{\lambda}\right)$ be a solution of the free boundary problem (2.19)(2.22) with $p, u \in C\left[0, R_{\lambda}\right] \cap C^{1}\left(0, R_{\lambda}\right)$. Then assertions (1)-(5) of Theorem 7.1 hold.

Proof. Indeed, in the proof of Theorem 7.1, the condition that $p(r)$ is differentiable at $r=0$ and $r=R_{\lambda}$ is used only to ensure that at these points $u(r) p^{\prime}(r)=0$ so that

$$
p(0)=\alpha(\lambda) \quad \text { and } \quad p\left(R_{\lambda}\right)=\alpha(1) .
$$

By Theorem 8.1 we see that (8.17) holds also if $p \in C\left[0, R_{\lambda}\right] \cap C^{1}\left(0, R_{\lambda}\right), u \in$ $C\left[0, R_{\lambda}\right] \cap C^{1}\left(0, R_{\lambda}\right)$ and $(p, u)$ satisfy $(2.19)-(2.22)$. Hence the desired assertion follows.

Definition 8.1. A pair of functions $p, u$ in $L^{\infty}[0, R]$ satisfying (8.1), (8.2) a.e. in $[0, R]$ is called a weak solution of $(2.19)-(2.21)$.

Theorem 8.1 asserts that if $(p, u)$ is a weak solution and $p \geq 0$, then it is also a classical solution, that is, $p \in C[0, R] \cap C^{1}(0, R], u \in C^{1}[0, R]$, and (2.19)-(2.21) 
hold. However, if we remove the condition $p \geq 0$, then the result may be different, as briefly discussed in the next paragraph.

The condition (8.3) was used to prove that $p\left(r_{1}-0\right)=\alpha\left(c_{\lambda}\left(r_{1}\right)\right)$ and similarly that $p\left(r_{1}+0\right)=\alpha\left(c_{\lambda}\left(r_{1}\right)\right)$, so that $p(r)$ is continuous at any boundary point $r_{1}$ of $O$. If $p(r)$ changes sign, then it may occur that

$$
p\left(r_{1}-0\right)=\alpha\left(c_{\lambda}\left(r_{1}\right)\right) \text { but } p\left(r_{1}+0\right)=\alpha_{-}\left(c_{\lambda}\left(r_{1}\right)\right)
$$

(or vice versa), where $\alpha_{-}(\lambda)$ is the negative root of (3.1) (see (7.9)). Thus a weak solution need not be a classical solution.

The following result supplements Theorem 8.1 in case $(p, u)$ is a weak solution and $p$ may change sign.

Theorem 8.3. Let $(p, u)$ and $(\bar{p}, \bar{u})$ be respectively a weak solution and a classical solution of the problem (2.19)-(2.21) with the same $\lambda, \bar{p} \geq 0$. Suppose that

$$
p(r)=\bar{p}(r), \quad u(r)=\bar{u}(r)
$$

for $0<r<\delta$, for some $\delta>0$. Then (8.18) holds for all $0<r<R$, where $R$ is equal to:

(i) the positive zero of $\bar{u}$ if $(\bar{p}, \bar{u})$ is a subsolution,

(ii) the blow-up point of $\bar{p}$ if $(\bar{p}, \bar{u})$ is an unbounded upper solution,

(iii) $R_{\lambda}$ if $(\bar{p}, \bar{u})$ is a bounded upper solution, or

(iv) the first positive zero of $\frac{d \bar{p}(r)}{d r}$ if $(\bar{p}, \bar{u})$ is a lower solution.

Proof. Let $\left(0, r_{0}\right)\left(r_{0}>0\right)$ be the largest open interval such that (8.18) holds for all $r \in\left(0, r_{0}\right)$. We claim that $r_{0} \geq R$. Indeed, if $r_{0}<R$, then, since (8.18) holds for all $r \in\left(0, r_{0}\right)$ and since $u(r)$ is a continuous function (by $\left.(8.1)\right)$ and $\bar{u}(r)<0$ for $0<r<R$, it follows that $u(r)<0$ for $0<r<r_{0}+\varepsilon$ (for some $\varepsilon>0 ; r_{0}+\varepsilon<R$ ). Thus, by the proof of Theorem 8.1,p(r) is continuous and $(p(r), u(r))$ is a classical solution for $0<r<r_{0}+\varepsilon$. By uniqueness of classical solutions we deduce that $p(r)=\bar{p}(r)$ and $u(r)=\bar{u}(r)$ for $0<r<r_{0}+\varepsilon$, which contradicts the maximality of $r_{0}$.

\section{Existence OF SUBsolutions}

In this section we prove that for any $\lambda$ less than, but near, $\lambda_{\infty}$, there exists a unique $\bar{\psi}=\bar{\psi}(\lambda)$ such that $\left(p_{\lambda \bar{\psi}}, u_{\lambda \bar{\psi}}\right)$ is a subsolution. In the next section we shall prove that for any $\lambda$ near 0 , there exists a unique $\bar{\psi}=\bar{\psi}(\lambda)$ such that $\left(p_{\lambda \bar{\psi}}, u_{\lambda \bar{\psi}}\right)$ is a supersolution. These facts will be used to prove the existence of a solution to the free boundary problem.

The following comparison lemma will play a crucial role:

Lemma 9.1. Let $\left(p_{1}, u_{1}\right)$ and $\left(p_{2}, u_{2}\right)$ be two different solutions of $(2.19)-(2.21)$ with the same $\lambda \in\left(0, \lambda_{\infty}\right)$, defined on the same interval $[0, R)\left(0<R \leq R_{\lambda}\right)$. Suppose that

$$
p_{1}(r)>p_{2}(r) \quad \text { and } \quad u_{1}(r)>u_{2}(r)
$$

in some small interval $0<r<\delta$. Suppose further that

$$
u_{1}(r)<0, \quad u_{2}(r)<0 \quad \text { and } \quad p_{2}^{\prime}(r)>0
$$

for $0<r<R$. Then (9.1) holds for all $0<r<R$. 
Proof. Assume that the assertion is not true. Then there exists an $r_{0} \in(0, R)$ such that (9.1) holds for $0<r<r_{0}$ and either (i) $p_{1}\left(r_{0}\right)>p_{2}\left(r_{0}\right), u_{1}\left(r_{0}\right)=u_{2}\left(r_{0}\right)$, or (ii) $p_{1}\left(r_{0}\right)=p_{2}\left(r_{0}\right), u_{1}\left(r_{0}\right)>u_{2}\left(r_{0}\right)$. (Note that the case $p_{1}\left(r_{0}\right)=p_{2}\left(r_{0}\right)$, $u_{1}\left(r_{0}\right)=u_{2}\left(r_{0}\right)$ cannot take place, by uniqueness of solutions.) In the first case we have $u_{1}^{\prime}\left(r_{0}\right)>u_{2}^{\prime}\left(r_{0}\right)$, by (2.19). On the other hand, from $u_{1}(r)>u_{2}(r)$ for $0<r<r_{0}$ and $u_{1}\left(r_{0}\right)=u_{2}\left(r_{0}\right)$, it follows that $u_{1}^{\prime}\left(r_{0}\right) \leq u_{2}^{\prime}\left(r_{0}\right)$, which is a contradiction. In the second case we have $u_{1}\left(r_{0}\right) p_{1}^{\prime}\left(r_{0}\right)=u_{2}\left(r_{0}\right) p_{2}^{\prime}\left(r_{0}\right)$, by $(2.21)$. Since $\left|u_{2}\left(r_{0}\right)\right|>\left|u_{1}\left(r_{0}\right)\right|$, we get

$$
\frac{p_{1}^{\prime}\left(r_{0}\right)}{p_{2}^{\prime}\left(r_{0}\right)}=\frac{u_{2}\left(r_{0}\right)}{u_{1}\left(r_{0}\right)}>1
$$

so that $p_{1}^{\prime}\left(r_{0}\right)>p_{2}^{\prime}\left(r_{0}\right)$. On the other hand, from $p_{1}(r)>p_{2}(r)$ for $0<r<r_{0}$ and $p_{1}\left(r_{0}\right)=p_{2}\left(r_{0}\right)$, it follows that $p_{1}^{\prime}\left(r_{0}\right) \leq p_{2}^{\prime}\left(r_{0}\right)$, which is again a contradiction. Hence the desired assertion follows.

Lemma 9.2. For any $\lambda \in\left(0, \lambda_{\infty}\right)$ the following hold:

(1) If for some $\bar{\psi} \in \mathbf{R},\left(p_{\lambda \bar{\psi}}, u_{\lambda \bar{\psi}}\right)$ is a subsolution, then for all $\psi>\bar{\psi},\left(p_{\lambda \psi}, u_{\lambda \psi}\right)$ is an unbounded upper solution, and for all $\psi<\bar{\psi},\left(p_{\lambda \psi}, u_{\lambda \psi}\right)$ is a lower solution.

(2) If for some $\bar{\psi} \in \mathbf{R},\left(p_{\lambda \bar{\psi}}, u_{\lambda \bar{\psi}}\right)$ is an upper solution, then for all $\psi>\bar{\psi}$, $\left(p_{\lambda \psi}, u_{\lambda \psi}\right)$ is also an upper solution; in particular, if for some $\bar{\psi} \in \mathbf{R},\left(p_{\lambda \bar{\psi}}, u_{\lambda \bar{\psi}}\right)$ is an unbounded upper solution, then for all $\psi>\bar{\psi},\left(p_{\lambda \psi}, u_{\lambda \psi}\right)$ is also an unbounded upper solution.

(3) If for some $\bar{\psi} \in \mathbf{R},\left(p_{\lambda \bar{\psi}}, u_{\lambda \bar{\psi}}\right)$ is a lower solution, then for all $\psi<\bar{\psi}$, $\left(p_{\lambda \psi}, u_{\lambda \psi}\right)$ is also a lower solution.

Proof. Assertion (2) follows quickly from Lemma 9.1. Indeed, from the expressions (5.43)-(5.46) and (5.56) near $r=0$ and the fact that $\omega=\omega(\lambda, \psi)$ is monotone increasing in $\psi$ it follows that, if $\psi>\bar{\psi}$, then

$$
p_{\lambda \psi}(r)>p_{\lambda \bar{\psi}}(r), \quad u_{\lambda \psi}(r)>u_{\lambda \bar{\psi}}(r)
$$

for $r$ near 0. If $\left(p_{\lambda \bar{\psi}}(r), u_{\lambda \bar{\psi}}(r)\right)$ is an upper solution, then $\frac{d}{d r} p_{\lambda \bar{\psi}}(r)>0$, so that by Lemma 9.1, the above inequalities hold also for all $r$ such that both $\left(p_{\lambda \psi}(r), u_{\lambda \psi}(r)\right)$ and $\left(p_{\lambda \bar{\psi}}(r), u_{\lambda \bar{\psi}}(r)\right)$ are well-defined.

To prove assertion (1), consider first the case $\psi>\bar{\psi}$. As before, (9.3) holds for all $r$ such that both $\left(p_{\lambda \psi}(r), u_{\lambda \psi}(r)\right)$ and $\left(p_{\lambda \bar{\psi}}(r), u_{\lambda \bar{\psi}}(r)\right)$ are well-defined. It follows that $\left(p_{\lambda \psi}, u_{\lambda \psi}\right)$ is either a subsolution or an upper solution. We claim that $\left(p_{\lambda \psi}, u_{\lambda \psi}\right)$ cannot be a subsolution. Indeed, if $\left(p_{\lambda \psi}, u_{\lambda \psi}\right)$ is a subsolution, then, by (9.3), the zero $\tilde{R}_{\lambda \psi}$ of $u_{\lambda \psi}(r)$ and the zero $\tilde{R}_{\lambda \bar{\psi}}$ of $u_{\lambda \bar{\psi}}(r)$ must satisfy the inequality $\tilde{R}_{\lambda \psi}<\tilde{R}_{\lambda \bar{\psi}}$. Since $\left(p_{\lambda \bar{\psi}}, u_{\lambda \bar{\psi}}\right)$ is a subsolution, $p_{\lambda \bar{\psi}}(r)>\alpha\left(c_{\lambda}(r)\right)$ for $0<r<\tilde{R}_{\lambda \bar{\psi}}$, so that

$$
p_{\lambda \psi}\left(\tilde{R}_{\lambda \psi}\right)=\alpha\left(c_{\lambda}\left(\tilde{R}_{\lambda \psi}\right)\right)<p_{\lambda \bar{\psi}}\left(\tilde{R}_{\lambda \psi}\right),
$$

which contradicts (9.3). Hence $\left(p_{\lambda \psi}, u_{\lambda \psi}\right)$ is an upper solution, and it is either bounded or unbounded. If it is bounded, then $u_{\lambda \psi}(r)<0$ for all $0<r \leq R_{\lambda}$, which is a contradiction because $u_{\lambda \psi}(r)>u_{\lambda \bar{\psi}}(r)=0$ at $r=\tilde{R}_{\lambda \bar{\psi}}$. Hence $\left(p_{\lambda \psi}, u_{\lambda \psi}\right)$ is an unbounded upper solution. Consider next the case $\psi<\bar{\psi}$. By assertion (2), $\left(p_{\lambda \psi}, u_{\lambda \psi}\right)$ cannot be an upper solution, and by the result we have just proved, $\left(p_{\lambda \psi}, u_{\lambda \psi}\right)$ cannot be a subsolution. It follows that $\left(p_{\lambda \psi}, u_{\lambda \psi}\right)$ must be a lower solution.

Finally, assertion (3) follows immediately from (1) and (2). 
Lemma 9.3. For any $\lambda \in\left(0, \lambda_{\infty}\right)$, the set

$$
\Omega_{1}(\lambda)=\left\{\psi \in \mathbf{R}:\left(p_{\lambda \psi}, u_{\lambda \psi}\right) \text { is an upper solution }\right\}
$$

is bounded from below.

Proof. If the assertion is not true, then, by Lemma 9.2 (2), for any $\psi \in \mathbf{R}$, $\left(p_{\lambda \psi}, u_{\lambda \psi}\right)$ is an upper solution. Using Lemma 9.1, we infer that, as $\psi$ decreases, both $p_{\lambda \psi}(r)$ and $u_{\lambda \psi}(r)$ decrease, whereas the interval of definition of $\left(p_{\lambda \psi}, u_{\lambda \psi}\right)$ increases (for unbounded upper solutions). Since $p_{\lambda \psi}(r)(\psi \in \mathbf{R})$ are all positive and, by (8.1),

$$
u_{\lambda \psi}(r) \geq C>-\infty, \quad C \text { independent of } \psi,
$$

the limits

$$
p=\lim _{\psi \rightarrow-\infty} p_{\lambda \psi}, \quad u=\lim _{\psi \rightarrow-\infty} u_{\lambda \psi}
$$

exist and, furthermore

$$
0 \leq p<p_{\lambda \psi}, \quad u<u_{\lambda \psi} \text { for all } \psi \in \mathbf{R} .
$$

On the other hand, replacing $(p, u)$ in (8.1), (8.2) with $\left(p_{\lambda \psi}, u_{\lambda \psi}\right)$ and letting $\psi \rightarrow$ $-\infty$, we see that $(p, u)$ is a weak solution of (2.19)-(2.21), and, by Theorem 8.1, $(p, u)$ is also a classical solution. It follows that there exists a $\psi_{0} \in \mathbf{R}$ such that $p=p_{\lambda \psi_{0}}$ and $u=u_{\lambda \psi_{0}}$, which contradicts (9.4). Hence the desired assertion follows.

Lemma 9.4. For any $\lambda \in\left(0, \lambda_{\infty}\right)$, the set

$$
\Omega_{2}(\lambda)=\left\{\psi \in \mathbf{R}:\left(p_{\lambda \psi}, u_{\lambda \psi}\right) \text { is a lower solution }\right\}
$$

is bounded from above.

Proof. If the assertion is false, then, by Lemma $9.2(3),\left(p_{\lambda \psi}, u_{\lambda \psi}\right)$ is a lower solution for any $\psi \in \mathbf{R}$. By Theorem 7.4 (3), the functions $\left\{p_{\lambda \psi}(r): \psi \in \mathbf{R}\right\}$ form a bounded subset of $L^{\infty}\left[0, R_{\lambda}\right]$. We can therefore find an unbounded increasing sequence $\left\{\psi_{n}\right\}_{n=1}^{\infty}$ such that the corresponding sequence $\left\{p_{\lambda \psi_{n}}\right\}_{n=1}^{\infty}$ is $*$-weakly convergent in $L^{\infty}\left[0, R_{\lambda}\right]$. Let $p$ denote the limit function. By $(8.1)$, the corresponding sequence $\left\{u_{\lambda \psi_{n}}\right\}_{n=1}^{\infty}$ is uniformly convergent on $\left[0, R_{\lambda}\right]$. Let $u$ denote the limit function. Replacing $(p, u)$ in (8.1), (8.2) with $\left(p_{\lambda \psi_{n}}, u_{\lambda \psi_{n}}\right)$ and letting $n \rightarrow \infty$, one easily finds that $(p, u)$ is a weak solution of $(2.19)-(2.21)$. We claim that there exists a $\bar{\psi} \in \mathbf{R}$ such that, for any $\psi>\bar{\psi}$,

$$
\frac{d}{d r} p_{\lambda \psi}(r)>0 \quad \text { for } 0<r<\delta_{\psi}
$$

for some $\delta_{\psi}>0$. Indeed, if $\sigma(\lambda)>1$, then by (5.43), (5.45) we have

$$
p(r)=\alpha(\lambda)+\frac{1}{2} \alpha_{2}(\lambda) r^{2}+o\left(r^{2}\right) \quad \text { as } r \rightarrow 0 .
$$

Since, by (4.10),

$$
\alpha_{2}(\lambda)=\frac{1}{\gamma_{2}(\lambda)}\left\{K_{P}^{\prime}(\lambda)+\left(K_{M}^{\prime}(\lambda)-K_{N}^{\prime}(\lambda)\right)-K_{M}^{\prime}(\lambda) \alpha^{2}(\lambda)\right\} c_{\lambda}^{\prime \prime}(\lambda)>0,
$$

we see that (9.5) actually holds for all $\psi \in \mathbf{R}$. If $\sigma(\lambda)=1$, then by (5.45) (taking $n=1$ ) we see that (9.5) also holds for all $\psi \in \mathbf{R}$, because by (5.22),

$$
\mu_{0}(\lambda)=\frac{1}{2 \beta(\lambda)}\left\{K_{P}^{\prime}(\lambda)+\left(K_{M}^{\prime}(\lambda)-K_{N}^{\prime}(\lambda)\right)-K_{M}^{\prime}(\lambda) \alpha^{2}(\lambda)\right\} c_{\lambda}^{\prime \prime}(\lambda)<0 .
$$


Finally, if $-1<\sigma(\lambda)<1$, then (9.5) follows from (5.43), (5.54) and (5.55), provided $\psi$ is sufficiently large.

Having proved (9.5), we can now apply Lemma 9.1 to deduce that, if $\psi^{\prime}>\psi>\bar{\psi}$, then

$$
p_{\lambda \psi^{\prime}}(r)>p_{\lambda \psi}(r), \quad u_{\lambda \psi^{\prime}}(r)>u_{\lambda \psi}(r)
$$

as long as $\frac{d}{d r} p_{\lambda \psi}(r)>0$. Assuming for simplicity that $\psi_{1}>\bar{\psi}$, we deduce, in particular, that

$$
p_{\lambda \psi_{n}}(r) \geq p_{\lambda \psi_{1}}(r) \text { for all } 0<r<\tilde{R} \text { and } n \geq 1,
$$

where $\tilde{R}$ is the first positive zero of $d p_{\lambda \psi_{1}} / d r$. It follows that

$$
p(r) \geq p_{\lambda \psi_{1}}(r) \geq 0 \text { for } 0 \leq r \leq \tilde{R}
$$

so that, by Theorem 8.1, $(p, u)$ is a classical solution on the interval $[0, \tilde{R}]$. By uniqueness of the solutions, we infer that there exists $\psi_{0} \in \mathbf{R}$ so that $(p, u)=$ $\left(p_{\lambda \psi_{0}}, u_{\lambda \psi_{0}}\right)$. Now take a positive integer $N$ large enough so that $\psi_{N}>\psi_{0}$, which ensures that, for some $\delta>0$,

$$
p_{\lambda \psi_{N}}(r)>p_{\lambda \psi_{0}}(r) \text { for } 0<r<\delta .
$$

But since

$$
p_{\lambda \psi_{n}}(r) \geq p_{\lambda \psi_{N}}(r) \quad \text { for } 0<r<\tilde{R}^{\prime} \text { and } n \geq N
$$

where $\tilde{R}^{\prime}$ is the first positive zero of $d p_{\lambda \psi_{N}} / d r$, we have, by taking the $*$-weak limit of the $p_{\lambda \psi_{n}}$,

$$
p_{\lambda \psi_{0}}(r) \geq p_{\lambda \psi_{N}}(r) \quad \text { for } 0 \leq r \leq \tilde{R}^{\prime},
$$

which is a contradiction. This completes the proof of the lemma.

Remark 9.1. Since $p_{\lambda \psi}(r)$ is uniformly bounded for absolutely large negative $\psi$, one can find a sequence $\psi_{m} \rightarrow-\infty$ such that $p_{\lambda \psi_{m}}(r) *$-weakly converges in $L^{\infty}\left[0, R_{\lambda}\right]$ and $u_{\lambda \psi_{m}}(r)$ uniformly converges for $0 \leq r \leq R_{\lambda}$. The limits $p(r), u(r)$ form a weak solution of (2.19)-(2.21). By similar arguments as in the above proof, one can show that this solution cannot be a classical solution such that $p(0)=\alpha(\lambda)$. Thus $p(0)=\alpha_{-}(\lambda)$, where $\alpha_{-}(\lambda)$ is the negative root of (3.1) (see (7.9)). One can further deduce that $p(r) \leq 0$ for all $0 \leq r \leq R_{\lambda}$.

Lemma 9.5. There exists a $\bar{\lambda} \in\left(0, \lambda_{\infty}\right)$ such that for all $\lambda \in\left(\bar{\lambda}, \lambda_{\infty}\right)$ the set

$$
\Omega_{3}(\lambda)=\left\{\psi \in \mathbf{R}:\left(p_{\lambda \psi}, u_{\lambda \psi}\right) \text { is a bounded upper solution }\right\}
$$

is empty.

Proof. For any $\lambda \in(0,1)$ we introduce the function

$$
w_{\lambda}(r)=\frac{1}{r^{2}} \int_{0}^{r}\left(-K_{D}\left(c_{\lambda}(\rho)\right)+K_{M}\left(c_{\lambda}(\rho)\right) \alpha\left(c_{\lambda}(\rho)\right)\right) \rho^{2} d \rho, \quad 0<r \leq R_{\lambda},
$$

and set $w_{\lambda}(0)=0$. It is clear that $w_{\lambda}$ depends continuously on $\lambda$ in the topology of $C\left[0, R_{\lambda}\right]$. Clearly,

$$
\frac{d}{d r}\left(r^{2} w_{\lambda}(r)\right)=3 r^{2} \beta\left(c_{\lambda}(r)\right), \quad 0 \leq r \leq R_{\lambda},
$$

where $\beta(\cdot)$ is the function defined in (3.6), so that by Lemma 3.2,

$$
w_{\lambda}(r)>0 \text { for } 0<r \leq R_{\lambda}, \quad \lambda_{\infty} \leq \lambda<1,
$$


and

$$
\begin{gathered}
w_{\lambda}^{\prime}(0)=\beta(\lambda)<0, \\
\frac{d}{d r}\left(w_{\lambda}^{\prime}(r)+\frac{2}{r} w_{\lambda}(r)\right)>0, \quad 0 \leq r \leq R_{\lambda},
\end{gathered}
$$

for $0<\lambda<\lambda_{\infty}$. Hence there exists a $\bar{\lambda} \in\left(0, \lambda_{\infty}\right)$ such that for $\lambda \in\left(\bar{\lambda}, \lambda_{\infty}\right)$,

$$
w_{\lambda}(r)<0 \text { for } 0<r<\hat{R}_{\lambda}, \quad w_{\lambda}(r)>0 \text { for } \hat{R}_{\lambda}<r \leq R_{\lambda},
$$

for some point $\hat{R}_{\lambda}$. We claim that, for $\lambda \in\left(\bar{\lambda}, \lambda_{\infty}\right)$, the set $\Omega_{3}(\lambda)$ is empty. Indeed, if this is not the case, then there exists a bounded upper solution $(p, u)$ for this $\lambda$, so that $p(r)>\alpha\left(c_{\lambda}(r)\right)$ for all $0<r \leq R_{\lambda}$. By (8.1), we then have $u(r)>w_{\lambda}(r)$ for $0<r \leq R_{\lambda}$. Therefore, $u(r)>0$ for $\hat{R}_{\lambda} \leq r \leq R_{\lambda}$. Since $u^{\prime}(0)=\beta(\lambda)<0$, so that $u(r)<0$ for $r$ near 0 , we see that $(p, u)$ is a subsolution, which is a contradiction.

Lemma 9.6. Given $\lambda \in\left(0, \lambda_{\infty}\right)$, there exists an entire solution of the problem (2.19)-(2.21) which is a subsolution if and only if the set $\Omega_{3}(\lambda)$ is empty.

Proof. If (2.19)-(2.21) has a subsolution, then by Lemma $9.2(1)$, the set $\Omega_{3}(\lambda)$ is empty. Suppose conversely that $\Omega_{3}(\lambda)$ is empty. By Lemmas 9.3 and 9.4, $\Omega_{1}(\lambda)$ and $\Omega_{2}(\lambda)$ are bounded, respectively, from below and from above, and by Lemma 9.2 (1) there is at most one $\psi$ such that $\left(p_{\lambda \psi}, u_{\lambda \psi}\right)$ is a subsolution. Consequently, $\Omega_{1}(\lambda)$ and $\Omega_{2}(\lambda)$ are both nonempty and, by Lemma 9.2 ,

$$
\inf \Omega_{1}(\lambda)=\sup \Omega_{2}(\lambda) \equiv \bar{\psi}
$$

We claim that $(p, u) \equiv\left(p_{\lambda \bar{\psi}}, u_{\lambda \bar{\psi}}\right)$ is a subsolution. Indeed, using Theorem 6.1, we easily see that the set $\Omega_{2}(\lambda)$ is open, so that $\bar{\psi} \notin \Omega_{2}(\lambda)$. To prove that $\bar{\psi} \notin \Omega_{1}(\lambda)$ we assume the converse: $\bar{\psi} \in \Omega_{1}(\lambda)$. Let $[0, R)$ be the domain of definition of $(p, u)$, so that

$$
\lim _{r \rightarrow R} p(r)=+\infty
$$

By the uniform boundedness of $\left\{p_{\lambda \psi}: \psi \in \Omega_{2}(\lambda)\right\}$, we can find an increasing sequence $\left\{\psi_{n}\right\}_{n=1}^{\infty}$ converging to $\bar{\psi}$, such that $p_{\lambda \psi_{n}}$ converges $*$-weakly in $L^{\infty}\left[0, R_{\lambda}\right]$ to some function $\bar{p} \in L^{\infty}\left[0, R_{\lambda}\right]$, and $u_{\lambda \psi_{n}}$ converges uniformly to some function $\bar{u} \in C\left[0, R_{\lambda}\right]$. Replacing $(p, u)$ in (8.1), (8.2) with $\left(p_{\lambda \psi_{n}}, u_{\lambda \psi_{n}}\right)$ and letting $n \rightarrow \infty$, one easily finds that $(\bar{p}, \bar{u})$ is a weak solution of $(2.19)-(2.21)$. Since $\lim _{n \rightarrow \infty} \psi_{n}=\bar{\psi}$, we conclude, as in the proof of Lemma 9.4, that $(p(r), u(r))=(\bar{p}(r), \bar{u}(r))$ for $r$ near 0 , so that, by Theorem 8.3 ,

$$
p(r)=\bar{p}(r), \quad u(r)=\bar{u}(r) \quad \text { for } 0<r<R .
$$

It follows that $p(r)$ is bounded for $r \in[0, R)$, which is a contradiction. Therefore, $\bar{\psi} \notin \Omega_{1}(\lambda)$.

Since we have proved that $\bar{\psi}$ does not belong to $\Omega_{1}(\lambda)$ nor to $\Omega_{2}(\lambda)$, it follows that $(p, u)$ must be a subsolution.

By Lemmas 9.5 and 9.6, we immediately get the following result:

Lemma 9.7. There exists a $\bar{\lambda} \in\left(0, \lambda_{\infty}\right)$ such that for any $\lambda \in\left(\bar{\lambda}, \lambda_{\infty}\right)$, there is a unique number $\bar{\psi}=\bar{\psi}(\lambda)$ such that $\left(p_{\lambda \bar{\psi}}, u_{\lambda \bar{\psi}}\right)$ is a subsolution. 


\section{EXISTENCE OF SOLUTIONS OF THE FREE BOUNDARY PROBLEM}

In this section we prove the existence of a solution of the free boundary problem (2.19)-(2.22). If for some $\lambda \in\left(0, \lambda_{\infty}\right)$ there is a $\bar{\psi} \in \mathbf{R}$ such that $\left(p_{\lambda \bar{\psi}}, u_{\lambda \bar{\psi}}\right)$ is a subsolution (resp. supersolution), then we say that $\lambda$ is a subsolution point (resp. supersolution point). Similarly, if for some $\lambda \in\left(0, \lambda_{\infty}\right)$ there exists $\bar{\psi} \in \mathbf{R}$ such that $\left(p_{\lambda \bar{\psi}}, u_{\lambda \bar{\psi}}, R_{\lambda}\right)$ is a solution of the free boundary problem $(2.19)-(2.22)$, then we say that $\lambda$ is a solution point. A subsolution that is not a solution of the free boundary problem will be called a strict subsolution, and the corresponding $\lambda$ will be called a strict subsolution point. We introduce the sets

$$
\begin{aligned}
& B_{1}=\left\{\lambda \in\left(0, \lambda_{\infty}\right): \lambda \text { is a strict subsolution point }\right\}, \\
& \tilde{B}_{1}=\left\{\lambda \in\left(0, \lambda_{\infty}\right): \lambda \text { is a subsolution point }\right\} \\
& B_{2}=\left\{\lambda \in\left(0, \lambda_{\infty}\right): \lambda \text { is a supersolution point }\right\}
\end{aligned}
$$

By Lemma 9.7 , the set $\tilde{B}_{1}$ is nonempty; in fact, the proof of Lemma 9.5 shows that $B_{1}$ is nonempty. Later on we shall prove that $B_{2}$ is nonempty.

Lemma 10.1. $\lambda$ is a supersolution point if and only if it is not a subsolution point; in other words,

$$
\tilde{B}_{1} \cup B_{2}=\left(0, \lambda_{\infty}\right), \quad \tilde{B}_{1} \cap B_{2}=\varnothing .
$$

Proof. Lemma 9.2 (1) shows that if $\lambda$ is a subsolution point, then $\lambda$ cannot be a supersolution point. To prove the converse, we note that if $\lambda$ is not a subsolution point, then the set $\Omega_{3}(\lambda)$ is nonempty (by Lemma 9.6) and is bounded below (by Lemma 9.3). Let

$$
\bar{\psi}=\inf \Omega_{3}(\lambda) .
$$

We claim that $(p, u)=\left(p_{\lambda \bar{\psi}}, u_{\lambda \bar{\psi}}\right)$ is a supersolution. Indeed, using Lemma 9.1 and Theorem 8.1, one readily finds that $p$ and $u$ are the monotone decreasing limits of $p_{\lambda \psi}$ and $u_{\lambda \psi}$, respectively, as $\psi \rightarrow \bar{\psi}+0, \psi \in \Omega_{3}(\lambda)$, so that they satisfy

$$
p(r)>\alpha\left(c_{\lambda}(r)\right), \quad p^{\prime}(r)>0, \quad u(r)<0 \text { for } 0<r<R_{\lambda},
$$

and $p\left(R_{\lambda}\right) \geq \alpha\left(c_{\lambda}\left(R_{\lambda}\right)\right)=1, u\left(R_{\lambda}\right)<0$. If $p\left(R_{\lambda}\right)>1$, then for sufficiently small $\delta>0$ we can find a corresponding $\varepsilon>0$ such that

$$
p(r)-\alpha\left(c_{\lambda}(r)\right) \geq \varepsilon, \quad u(r) \leq-\varepsilon
$$

for all $\delta \leq r \leq R_{\lambda}$. By Theorem 6.1 and the standard ODE theory, it follows that if $\psi$ is sufficiently close to $\bar{\psi}$, then

$$
p_{\lambda \psi}(r)-\alpha\left(c_{\lambda}(r)\right) \geq \frac{1}{2} \varepsilon, \quad u_{\lambda \psi}(r) \leq-\frac{1}{2} \varepsilon
$$

for $\delta \leq r \leq R_{\lambda}$, so that if also $\psi<\bar{\psi}$, then $\psi \in \Omega_{3}(\lambda)$, which is a contradiction. Hence $p\left(R_{\lambda}\right)=1$, and $(p, u)$ is a supersolution.

In the sequel we need to consider limits of sequences of functions $\left(p_{\lambda_{n} \psi}, u_{\lambda_{n} \psi}\right)$ $(n=1,2, \cdots)$. Since these functions are not defined on a common interval, it will be convenient to make a transformation of variables $\left(r, p, u, c_{\lambda}\right) \rightarrow\left(\bar{r}, \bar{p}, \bar{u}, \bar{c}_{\lambda}\right)$ :

$$
\bar{r}=\frac{r}{R_{\lambda}}, \quad \bar{u}(\bar{r})=\frac{u\left(\bar{r} R_{\lambda}\right)}{R_{\lambda}}, \quad \bar{p}(\bar{r})=p\left(\bar{r} R_{\lambda}\right), \quad \bar{c}_{\lambda}(\bar{r})=c_{\lambda}\left(\bar{r} R_{\lambda}\right) .
$$

It is easy to verify that equations (2.19)-(2.21) are invariant under this change of variables, but the interval $\left[0, R_{\lambda}\right]$ is changed into the unit interval $[0,1]$. Clearly, all 
results established in previous sections are still valid for the transformed problem. In the sequel we shall always write, for brevity, the variables $\bar{r}, \bar{p}, \bar{u}$ and $\bar{c}_{\lambda}$ as respectively $r, p, u$ and $c_{\lambda}$. It should be noted that the values $p(1), u(1)$ in the new variables are respectively equal to the values $p\left(R_{\lambda}\right)$ and $u\left(R_{\lambda}\right) / R_{\lambda}$ in the old variables.

Lemma 10.2. The sets $B_{1}, B_{2}$ are open.

Proof. To prove that $B_{1}$ is open we assume the converse, that is, there exists a point $\tilde{\lambda} \in B_{1}$ that is the limit of a sequence of points $\left\{\lambda_{m}\right\}_{m=1}^{\infty}$ that are not strict subsolution points. By Lemma 10.1, for each $\lambda_{m}$ we have a corresponding $\left(p_{m}, u_{m}\right)$ that is either a supersolution or a solution of the free boundary problem. In both cases we have, for each $m$,

$$
\alpha\left(c_{\lambda_{m}}(r)\right) \leq p_{m}(r) \leq 1, \quad p_{m}^{\prime}(r) \geq 0, \quad u_{m}(r) \leq 0 \quad \text { for } 0 \leq r \leq 1 .
$$

It follows that $\left\{p_{m}\right\}_{m=1}^{\infty}$ has a subsequence that is $*$-weakly convergent in $L^{\infty}[0,1]$. For simplicity we assume that $\left\{p_{m}\right\}_{m=1}^{\infty}$ is such a subsequence, and denote by $p$ the limit function. Then $\left\{u_{m}\right\}_{m=1}^{\infty}$ converges uniformly to a function $u \in C[0,1]$. Clearly,

$$
\alpha\left(c_{\tilde{\lambda}}(r)\right) \leq p(r) \leq 1, \quad u(r) \leq 0 \text { for } 0 \leq r \leq 1,
$$

and $p(r)$ is monotone nondecreasing for $0 \leq r \leq 1$. On the other hand, replacing $\lambda, p$ and $u$ in (8.1), (8.2) with, respectively, $\lambda_{m}, p_{m}$ and $u_{m}$ and letting $m \rightarrow \infty$, we see that $(p, u)$ is a weak solution of $(2.19)-(2.21)$ with respect to $\lambda=\tilde{\lambda}$. Since $p \geq 0,(p, u)$ is a classical solution, by Theorem 8.1, so that the above properties of $(p, u)$ imply that

$$
\alpha\left(c_{\tilde{\lambda}}(r)\right)<p(r)<1, \quad p^{\prime}(r)>0, \quad u(r)<0 \text { for } 0<r<1,
$$

and $p(1)=1$. Furthermore, we have either $u(1)=0$ or $u(1)<0$. Clearly, in the first case $(p, u)$ is a solution of the free boundary problem, and in the second case $(p, u)$ is a supersolution, so that in either case $\tilde{\lambda} \neq B_{1}$, which is a contradiction. Hence the set $B_{1}$ is open.

Next we prove that $B_{2}$ is open. Suppose that $\tilde{\lambda} \in B_{2}$ and let $\left(\tilde{p}_{\tilde{\lambda}}, \tilde{u}_{\tilde{\lambda}}\right)=$ $\left(p_{\tilde{\lambda} \tilde{\psi}}, u_{\tilde{\lambda} \tilde{\psi}}\right)$ be the corresponding supersolution. Then we have

$$
\tilde{p}_{\tilde{\lambda}}(r)>\alpha\left(c_{\tilde{\lambda}}(r)\right), \quad \tilde{p}_{\tilde{\lambda}}^{\prime}(r)>0, \quad \tilde{u}_{\tilde{\lambda}}(r)<0 \text { for } 0<r<1,
$$

and $\tilde{p}_{\tilde{\lambda}}(1)=1, \tilde{u}_{\tilde{\lambda}}(1)<0$. It follows by Theorem 6.1 and Lemma 9.1 that we can find a $\hat{\psi}>\tilde{\psi}$ sufficiently near $\tilde{\psi}$ such that

$$
p_{\tilde{\lambda} \hat{\psi}}(r)>\alpha\left(c_{\tilde{\lambda}}(r)\right), \quad u_{\tilde{\lambda} \hat{\psi}}(r)<0 \text { for } 0<r \leq 1
$$

(and consequently also $p_{\tilde{\lambda} \hat{\psi}}^{\prime}(r)>0$ for all $0<r \leq 1$ ). This implies that for any $\delta>0$ sufficiently small, there exists a corresponding $\varepsilon>0$ such that

$$
p_{\tilde{\lambda} \hat{\psi}}(r)-\alpha\left(c_{\tilde{\lambda}}(r)\right) \geq \varepsilon, \quad u_{\tilde{\lambda} \hat{\psi}}(r) \leq-\varepsilon \text { for } \delta \leq r \leq 1
$$

By Theorem 6.1 , it follows that for $(\lambda, \psi)$ sufficiently close to $(\tilde{\lambda}, \hat{\psi})$,

$$
p_{\lambda \psi}(r)-\alpha\left(c_{\lambda}(r)\right) \geq \frac{1}{2} \varepsilon, \quad u_{\lambda \psi}(r) \leq-\frac{1}{2} \varepsilon \quad \text { for } \delta \leq r \leq 1
$$

which implies that $\left(p_{\lambda \psi}, u_{\lambda \psi}\right)$ is a bounded upper solution. Hence for $\lambda$ in a small neighborhood of $\tilde{\lambda}$ the set $\Omega_{3}(\lambda)$ is nonempty and, therefore, by Lemma 9.6, these $\lambda$ 's belong to $B_{2}$, so that $B_{2}$ is open. 
When $\lambda=0$, the problem (2.19)-(2.21) (in the new variables) becomes

$$
\begin{gathered}
u^{\prime}(r)+\frac{2}{r} u(r)=-K_{D}(0)(1-p(r)), \quad 0<r<1, \\
u(0)=0, \\
u(r) p^{\prime}(r)=-\left\{\left(K_{Q}(0)-K_{D}(0)\right)+K_{D}(0) p(r)\right\} p(r), \quad 0<r<1 .
\end{gathered}
$$

The set of all solutions of (10.2)-(10.4) is characterized by the following lemma.

Lemma 10.3. For every $\omega \in \mathbf{R},(10.2)-(10.4)$ has a unique solution satisfying

$$
\begin{gathered}
p(r)=\alpha(0)+\omega r^{1+\sigma(0)}+o\left(r^{1+\sigma(0)}\right), \\
u(r)=\beta(0) r+\frac{\omega K_{D}(0)}{4+\sigma(0)} r^{2+\sigma(0)}+o\left(r^{2+\sigma(0)}\right)
\end{gathered}
$$

as $r \rightarrow 0$; furthermore, (i) if $\omega=0$, then

$$
p(r)=\alpha(0), \quad u(r)=\beta(0) r
$$

for all $0 \leq r \leq 1$; (ii) if $\omega>0$, then $(p, u)$ is an upper solution; (iii) if $\omega<0$, then $(p, u)$ is a lower solution.

Proof. The formula (10.5) and the uniqueness follow as in the proofs of Theorems 5.3 and 5.4. By direct computation one finds that $\left(p_{0}(r), u_{0}(r)\right)=(\alpha(0), \beta(0) r)$ is a solution of (10.2)-(10.4), so that there exists $\omega \in \mathbf{R}$ such that it coincides with the solution given by (10.5), and clearly $(p, u)=\left(p_{0}, u_{0}\right)$ for $\omega=0$. Next, noticing that $1+\sigma(0)>0$, we see that $p^{\prime}(r)<0$ for $r$ near 0 if $\omega<0$, so that $(p, u)$ is a lower solution if $\omega<0$.

Consider next the case $\omega>0$. Since $p^{\prime}(r)=(1+\sigma(0)) \omega r^{\sigma(0)}+o\left(r^{\sigma(0)}\right)($ as $r \rightarrow 0)$ and $u(0)=\beta(0)<0$, we can find a number $\delta>0$ such that

$$
p^{\prime}(r)>0, \quad u(r)<0
$$

for $0<r<\delta$. Let $(0, R)$ be the largest open interval such that (10.6) holds for $r \in(0, R)$. Then either $\lim _{r \rightarrow R} p(r)=+\infty$ or $\lim _{r \rightarrow R} p(r)=a$ for some $\alpha(0)<a<\infty$. In the first case $(p, u)$ is clearly an unbounded upper solution. In the second case the right-hand side of (10.2) is bounded, so that $b \equiv \lim _{r \rightarrow R} u(r)$ exists, and clearly $b \leq 0$. If $b<0$ and $R<1$, then we can extend $(p, u)$ to a larger interval such that $u(r)<0$ in this interval. Also $p^{\prime}(r)>0$ in this interval, for otherwise, by (10.4), $p(\bar{r})=\alpha(0)$ at the first point $\bar{r}$ where $p^{\prime}(\bar{r})=0$, which is a contradiction since $p(0)=\alpha(\lambda)$ and $p(r)$ is strictly increasing in $0 \leq r \leq \bar{r}$. Hence (10.6) still holds on this interval, which is contrary to the maximality of $(0, R)$. Thus either $b<0$ and $R=1$, or $b=0$. In the first case $(p, u)$ is a bounded upper solution. If we prove that the second case cannot occur, then the proof that $(p, u)$ is an upper solution (when $\omega>0$ ) will be completed. Suppose $b=0$. Then by (8.1), (8.2) and l'Hospital's rule,

$$
\begin{aligned}
a & =\lim _{r \rightarrow R} p(r)=\lim _{r \rightarrow R} \frac{\int_{0}^{r} K_{Q}(0) p(\rho) \rho^{2} d \rho}{\int_{0}^{r} K_{D}(0)(1-p(\rho)) \rho^{2} d \rho} \\
& =\lim _{r \rightarrow R} \frac{K_{Q}(0) p(r)}{K_{D}(0)(1-p(r))}=\frac{K_{Q}(0) a}{K_{D}(0)(1-a)},
\end{aligned}
$$

so that $a=\alpha(0)$, which is a contradiction. 
Lemma 10.4. The set $B_{2}$ is nonempty. More precisely, there exists a $\hat{\lambda} \in(0,1)$ such that $(0, \hat{\lambda}) \subset B_{2}$.

Proof. If the assertion is not true, then we can find a monotone decreasing sequence $\left\{\lambda_{m}\right\}_{m=1}^{\infty}$ converging to 0 such that each $\lambda_{m}$ is a subsolution point. Let $\left(p_{m}, u_{m}\right)$ be the corresponding sequence of subsolutions. Since $\left\{p_{m}\right\}_{m=1}^{\infty}$ is uniformly bounded, we may assume that $p_{m}$ *-weakly converges to some function $p \in L^{\infty}[0,1]$. It follows that $u_{m}$ uniformly converges to some function $u \in C[0,1]$. By a similar argument as before, we easily infer that $(p, u)$ is a solution of the problem (10.2)(10.4) with $p(r)$ monotone increasing. Using Lemma 10.3, we conclude that $(p, u)$ either is a bounded upper solution or is equal to $\left(p_{0}, u_{0}\right)$. It follows that $u(1)<0$. On the other hand, since $\left(p_{m}, u_{m}\right)$ are subsolutions, we have $u_{m}(1) \geq 0$, so that also $u(1) \geq 0$, which is a contradiction.

Proof of Theorem 2.1. The sets $B_{1}, B_{2}$ are both open (by Lemma 10.2), nonempty (by Lemmas 9.7 and 10.4), and they are disjoint (by Lemma 10.1). Hence

$$
\left(0, \lambda_{\infty}\right) \neq B_{1} \cup B_{2} .
$$

Recalling (10.1), we conclude that $\tilde{B}_{1} \backslash B_{2} \neq \varnothing$, so that there exists at least one solution point.

\section{UNIQUENESS OF THE SOLUTION}

In this section we prove the uniqueness of the solution of the free boundary problem (2.1)-(2.6). We assume that there exist two different solutions $\left(c_{1}, p_{1}, u_{1}, R_{1}\right)$ and $\left(c_{2}, p_{2}, u_{2}, R_{2}\right)$, and derive a contradiction.

By Lemma $9.2, R_{1} \neq R_{2}$, and we may take $R_{1}<R_{2}$. Introducing the functions

$$
\bar{c}_{i}(r)=c_{i}\left(r R_{i}\right), \quad \bar{p}_{i}(r)=p_{i}\left(r R_{i}\right), \quad \bar{u}_{i}(r)=\frac{u_{i}\left(r R_{i}\right)}{R_{i}}, \quad 0 \leq r \leq 1, \quad i=1,2,
$$

we find that the $\left(\bar{c}_{i}, \bar{p}_{i}, \bar{u}_{i}\right)$ 's satisfy the system of equations

$\bar{u}_{i}(r) \bar{p}_{i}^{\prime}(r)=K_{P}\left(\bar{c}_{i}(r)\right)+\left(K_{M}\left(\bar{c}_{i}(r)\right)-K_{N}\left(\bar{c}_{i}(r)\right)\right) \bar{p}_{i}(r)-K_{M}\left(\bar{c}_{i}(r)\right) \bar{p}_{i}^{2}(r), 0<r<1$,

$$
\bar{u}_{i}(1)=0 \text {. }
$$

Since $R_{1}<R_{2}$, from (11.1) and (11.2) we get, by comparison, that

$$
\bar{c}_{1}(r)>\bar{c}_{2}(r) \text { for } 0 \leq r<1
$$

and, by the maximum principle, that

$$
\bar{c}_{1}^{\prime}(1)<\bar{c}_{2}^{\prime}(1) .
$$

Clearly

$$
\bar{p}_{1}(1)=\bar{p}_{2}(1)=1,
$$


so that, by (11.3),

$$
\bar{u}_{1}^{\prime}(1)=\bar{u}_{2}^{\prime}(1)=K_{B}(1)
$$

and, by (7.4),

$$
\begin{aligned}
\bar{p}_{i}^{\prime}(1) & =\frac{\left\{K_{P}^{\prime}(1)+\left(K_{M}^{\prime}(1)-K_{N}^{\prime}(1)\right) \bar{p}_{i}(1)-K_{M}^{\prime}(1) \bar{p}_{i}^{2}(1)\right\} \bar{c}_{i}^{\prime}(1)}{\bar{u}_{i}^{\prime}(1)+2 K_{M}(1) \bar{p}_{i}(1)-\left(K_{M}(1)-K_{N}(1)\right)} \\
& =\frac{-K_{Q}^{\prime}(1) \bar{c}_{i}^{\prime}(1)}{2 K_{B}(1)+K_{P}(1)} \equiv A \bar{c}_{i}^{\prime}(1),
\end{aligned}
$$

where $A>0$. Recalling (11.8), we conclude that

$$
\bar{p}_{1}^{\prime}(1)<\bar{p}_{2}^{\prime}(1) .
$$

From (11.9) and (11.11) it follows that there exists a $\delta>0$ such that

$$
\bar{p}_{1}(r)>\bar{p}_{2}(r) \text { for } 1-\delta<r<1 .
$$

Let $\left(r_{0}, 1\right)$ be the largest open interval such that

$$
\bar{p}_{1}(r)>\bar{p}_{2}(r) \text { for } r_{0}<r<1 ; \quad 0 \leq r_{0}<1 .
$$

By (11.3) we have

$$
\bar{u}_{i}(r)=\frac{1}{r^{2}} \int_{0}^{r}\left\{-K_{D}\left(\bar{c}_{i}(\rho)\right)+K_{M}\left(\bar{c}_{i}(\rho)\right) \bar{p}_{i}(\rho)\right\} \rho^{2} d \rho, \quad 0<r \leq 1 .
$$

Since the function $-K_{D}(c)+K_{M}(c) \alpha$ is strictly monotone increasing in both $c$ and $\alpha$, using (11.7) and (11.12) we infer that

$-K_{D}\left(\bar{c}_{1}(\rho)\right)+K_{M}\left(\bar{c}_{1}(\rho)\right) \bar{p}_{1}(\rho)>-K_{D}\left(\bar{c}_{2}(\rho)\right)+K_{M}\left(\bar{c}_{2}(\rho)\right) \bar{p}_{2}(\rho)$ for $r_{0}<r<1$.

Thus, if $r_{0}=0$, then, by (11.13), $\bar{u}_{1}(1)>\bar{u}_{2}(1)$, which is a contradiction to (11.6). It follows that $r_{0}>0$, and then

$$
\bar{p}_{1}\left(r_{0}\right)=\bar{p}_{2}\left(r_{0}\right)
$$

which implies, by (11.12), that

$$
\bar{p}_{1}^{\prime}\left(r_{0}\right) \geq \bar{p}_{2}^{\prime}\left(r_{0}\right)
$$

Since

$$
\int_{0}^{1}\left\{-K_{D}\left(\bar{c}_{i}(\rho)\right)+K_{M}\left(\bar{c}_{i}(\rho)\right) \bar{p}_{i}(\rho)\right\} \rho^{2} d \rho=\bar{u}_{i}(1)=0,
$$

(11.13) gives

$$
\bar{u}_{i}\left(r_{0}\right)=-\frac{1}{r_{0}^{2}} \int_{r_{0}}^{1}\left\{-K_{D}\left(\bar{c}_{i}(\rho)\right)+K_{M}\left(\bar{c}_{i}(\rho)\right) \bar{p}_{i}(\rho)\right\} \rho^{2} d \rho,
$$

so that, by (11.14),

$$
\bar{u}_{1}\left(r_{0}\right)<\bar{u}_{2}\left(r_{0}\right) .
$$

Combining this with (11.16) and recalling that $\bar{u}_{i}<0, \bar{p}_{i}^{\prime}>0$ (by Theorem 7.1), we conclude that

$$
\bar{u}_{1}\left(r_{0}\right) \bar{p}_{1}^{\prime}\left(r_{0}\right)<\bar{u}_{2}\left(r_{0}\right) \bar{p}_{2}^{\prime}\left(r_{0}\right) .
$$

On the other hand, since the function

$$
K_{P}(c)+\left(K_{M}(c)-K_{N}(c)\right) \alpha-K_{M}(c) \alpha^{2}=K_{P}(c)(1-\alpha)+K_{M}(c) \alpha(1-\alpha)-K_{Q}(c) \alpha
$$


is strictly monotone increasing in $c$ (for fixed $0<\alpha<1$ ), we infer from (11.5), (11.7) and (11.15) that

$$
\bar{u}_{1}\left(r_{0}\right) \bar{p}_{1}^{\prime}\left(r_{0}\right)>\bar{u}_{2}\left(r_{0}\right) \bar{p}_{2}^{\prime}\left(r_{0}\right)
$$

which is a contradiction.

Remark 11.1. The proof of the existence part of Theorem 2.1 shows that if $\lambda_{1}$ is a supersolution point and $\lambda_{2}$ is a subsolution point, then there exists a solution point of the free boundary problem in the interval with endpoints $\lambda_{1}, \lambda_{2}$. Furthermore, points $\lambda_{1}$ near 0 are supersolution points, and points $\lambda_{2}$ near $\lambda_{\infty}\left(\lambda_{2}<\lambda_{\infty}\right)$ are subsolution points. This fact, combined with the uniqueness part of Theorem 2.1, implies the following: If $\lambda_{*}$ is the unique solution point of the free boundary problem, then every $\lambda \in\left(0, \lambda_{*}\right)$ is a supersolution point, and every $\lambda \in\left(\lambda_{*}, \lambda_{\infty}\right)$ is a subsolution point.

Remark 11.2. The assumption $K_{D}(1)=K_{Q}(1)=0$ used throughout the paper can be weakened as follows:

$$
K_{D}(1) \geq 0, \quad K_{Q}(1) \geq 0 \quad \text { and } K_{Q}(1)<K_{B}(1) .
$$

In this case, $\alpha(1) \leq 1$, so that for a solution point $\lambda, u\left(R_{\lambda}\right)=0$ and $p\left(R_{\lambda}\right)=$ $\alpha(1) \leq 1$, rather than $p\left(R_{\lambda}\right)=1$ as in Theorem 2.1. Other than this and other similar differences, all the results of $\S \S 4-11$ hold with minor changes; for example, in Theorem 7.2 (a) one replaces $p(R)=1$ by $p(R) \leq 1$, and in $\S 11$ one replaces (11.10) by

$$
\bar{u}_{1}^{\prime}(1)=\bar{u}_{2}^{\prime}(1)=K_{B}(1) \alpha(1) .
$$

12. Appendix: The proofs of (5.30) and (6.18)-(6.19)

Lemma 12.1. Let $\bar{\lambda} \in\left(0, \lambda_{\infty}\right)$ and $\sigma(\bar{\lambda}) \geq n$, where $n$ is an integer $\geq 2$. Let $\lambda$ be a number in a small neighborhood of $\bar{\lambda}$. Assume that

$$
P(r)=\sum_{m=2}^{n} \frac{\alpha_{m}(\lambda)}{m !} r^{m-1}+v(r), \quad U(r)=\sum_{m=3}^{n+1} \frac{\beta_{m}(\lambda)}{m !} r^{m-1}+w(r) .
$$

Then

$$
\begin{aligned}
& (\beta(\lambda)+U(r))\left(f_{\lambda}(r, P(r), U(r))-\sum_{m=0}^{n-1} \mu_{m}(\lambda) r^{m}\right) \\
& \quad=r^{n} y(r)+z_{1}(r) w(r)+r z_{2}(r) v(r)-K_{M}\left(c_{\lambda}(r)\right) v^{2}(r),
\end{aligned}
$$

where

$$
|y(r)| \leq C, \quad\left|z_{1}(r)\right| \leq C, \quad\left|z_{2}(r)\right| \leq C,
$$

and $C$ is a constant independent of $v, w$ and $\lambda$.

Proof. Let $p(r)=\alpha(\lambda)+r P(r)$. Since $\alpha_{1}(\lambda)=0, \beta_{1}(\lambda)=\beta(\lambda)$ and $\beta_{2}(\lambda)=0$, we have

$$
\begin{gathered}
p(r)=\sum_{m=0}^{n} \frac{\alpha_{m}(\lambda)}{m !} r^{m}+r v(r) \equiv p_{n}(r)+r v(r), \\
\beta(\lambda)+U(r)=\sum_{m=1}^{n+1} \frac{\beta_{m}(\lambda)}{m !} r^{m-1}+w(r) .
\end{gathered}
$$


By (5.11), (5.12), (5.13) and (5.8) we see that

$$
\begin{aligned}
(\beta(\lambda)+ & U(r)) f_{\lambda}(r, P(r), U(r)) \\
= & -K_{M}\left(c_{\lambda}(r)\right) P^{2}(r)-(1 / r) g_{\lambda}(r) P(r)+\left(h_{\lambda}(r) / r^{2}\right) \\
& +\left(g_{\lambda}(0) / \beta(\lambda)\right)(1 / r)(\beta(\lambda)+U(r)) P(r) \\
= & \left(1 / r^{2}\right)\left\{K_{P}\left(c_{\lambda}(r)\right)+\left(K_{M}\left(c_{\lambda}(r)\right)-K_{N}\left(c_{\lambda}\right)\right) p(r)-K_{M}\left(c_{\lambda}(r)\right) p^{2}(r)\right\} \\
& -(\sigma(\lambda)+1)(1 / r)(\beta(\lambda)+U(r)) P(r) \\
\equiv & I_{1}-I_{2} .
\end{aligned}
$$

Clearly,

$$
\begin{aligned}
I_{1}= & \left(1 / r^{2}\right)\left\{K_{P}\left(c_{\lambda}(r)\right)+\left(K_{M}\left(c_{\lambda}(r)\right)-K_{N}\left(c_{\lambda}\right)\right) p_{n}(r)-K_{M}\left(c_{\lambda}(r)\right) p_{n}^{2}(r)\right\} \\
& +(1 / r)\left\{\left(K_{M}\left(c_{\lambda}(r)\right)-K_{N}\left(c_{\lambda}(r)\right)\right.\right. \\
& \left.\left.-2 K_{M}\left(c_{\lambda}(r)\right) p_{n}(r)\right) v(r)-K_{M}\left(c_{\lambda}(r)\right) \cdot r v^{2}(r)\right\} \\
\equiv & I_{11}+I_{12} .
\end{aligned}
$$

Using Taylor's expansions of the functions $K_{P}\left(c_{\lambda}(r)\right), K_{M}\left(c_{\lambda}(r)\right)$ and $K_{N}\left(c_{\lambda}(r)\right)$ up to order $n$ about $r=0$, and (5.19), (5.21), and recalling (3.1) and the relations

$$
k_{i}^{(1)}(\lambda)=\left.\frac{\partial}{\partial r} K_{i}\left(c_{\lambda}(r)\right)\right|_{r=0}=K_{i}^{\prime}(\lambda) c_{\lambda}^{\prime}(0)=0, \quad i=D, M, N, P
$$

we compute that

$$
\begin{aligned}
I_{11}= & \sum_{m=2}^{n+1} \frac{k_{P}^{(m)}(\lambda)}{m !} r^{m-2}+\left(\sum_{m=2}^{n+1} \frac{k_{M}^{(m)}(\lambda)-k_{N}^{(m)}(\lambda)}{m !} r^{m-2}\right)\left(\sum_{m=0}^{n} \frac{\alpha_{m}(\lambda)}{m !} r^{m}\right) \\
& -\left(\sum_{m=2}^{n+1} \frac{k_{M}^{(m)}(\lambda)}{m !} r^{m-2}\right)\left(\sum_{m=0}^{n} \frac{\alpha_{m}(\lambda)}{m !} r^{m}\right)^{2} \\
& +\left(K_{M}(\lambda)-K_{N}(\lambda)\right)\left(\sum_{m=2}^{n} \frac{\alpha_{m}(\lambda)}{m !} r^{m-2}\right) \\
& -K_{M}(\lambda)\left(2 \alpha(\lambda) \sum_{m=2}^{n} \frac{\alpha_{m}(\lambda)}{m !} r^{m-2}\right. \\
& \left.+r^{-2}\left(\sum_{m=2}^{n} \frac{\alpha_{m}(\lambda)}{m !} r^{m}\right)^{2}\right)+r^{n} y_{1}(r),
\end{aligned}
$$

or, by expanding the products,

$$
\begin{aligned}
I_{11}= & \sum_{m=0}^{n-1} \frac{r^{m}}{(m+2) !}\left\{k_{P}^{m+2}(\lambda)+\sum_{j=2}^{m+2}\left(\begin{array}{c}
m+2 \\
j
\end{array}\right)\left(k_{M}^{(j)}(\lambda)-k_{N}^{(j)}(\lambda)\right) \alpha_{m+2-j}(\lambda)\right. \\
& -\sum_{j=2}^{m+2} \sum_{k=0}^{m-j+2}\left(\begin{array}{c}
m+2 \\
j
\end{array}\right)\left(\begin{array}{c}
m-j+2 \\
k
\end{array}\right) k_{M}^{(j)}(\lambda) \alpha_{k}(\lambda) \alpha_{m-j-k+2}(\lambda) \\
& \left.-K_{M}(\lambda) \sum_{j=2}^{m}\left(\begin{array}{c}
m+2 \\
j
\end{array}\right) \alpha_{j}(\lambda) \alpha_{m-j+2}(\lambda)\right\} \\
& +\left(\left(K_{M}(\lambda)-K_{N}(\lambda)\right)-2 K_{M}(\lambda) \alpha(\lambda)\right)\left(\sum_{m=2}^{n} \frac{\alpha_{m}(\lambda)}{m !} r^{m-2}\right)+r^{n} y_{2}(r)
\end{aligned}
$$


so that, by the relation

$$
K_{M}(\lambda)-K_{N}(\lambda)-2 K_{M}(\lambda) \alpha(\lambda)=(\sigma(\lambda)+1) \beta(\lambda)
$$

(which follows from (3.8)) and the definition of $\mu_{m}$ (see (5.21) and (5.19)),

$$
\begin{aligned}
I_{11}= & \beta(\lambda) \sum_{m=0}^{n-1} \mu_{m}(\lambda) r^{m}+\sum_{m=1}^{n-1} \frac{r^{m}}{(m+2) !} \sum_{j=2}^{m+1}\left(\begin{array}{c}
m+2 \\
j
\end{array}\right) \beta_{j}(\lambda) \alpha_{m-j+3}(\lambda) \\
& +(\sigma(\lambda)+1) \beta(\lambda) \cdot \frac{1}{r}(P(r)-v(r))+r^{n} y_{3}(r) \\
\equiv & J_{1}+J_{2}+J_{3}+J_{4},
\end{aligned}
$$

where $y_{i}(r)(i=1,2,3)$ are functions depending only on $\alpha_{m}(\lambda)(0 \leq m \leq n), K_{P}$, $K_{M}, K_{N}$ and $c_{\lambda}$, so that they are uniformly bounded for all $\lambda$ near $\bar{\lambda}$. Later on we shall also use $y_{4}(r), z_{1}(r), z_{2}(r)$ to denote various functions possessing similar properties. Since

$$
\begin{aligned}
& \left(\sum_{j=2}^{n+1} \frac{\beta_{j}(\lambda)}{j !} r^{j-1}\right)\left(\sum_{k=1}^{n-1} \frac{\alpha_{k+1}(\lambda)}{k !} r^{k-1}\right) \\
& \quad=\left(\sum_{j=1}^{n} \frac{\beta_{j+1}(\lambda)}{(j+1) !} r^{j}\right)\left(\sum_{k=0}^{n-2} \frac{\alpha_{k+2}(\lambda)}{(k+1) !} r^{k}\right) \\
& \quad=\sum_{j=1}^{n} \sum_{k=0}^{n-2} \frac{r^{j+k}}{(j+k+2) !} \cdot \frac{(j+k+2) !}{(j+1) !(k+1) !} \cdot \beta_{j+1}(\lambda) \alpha_{k+2}(\lambda) \\
& =\sum_{m=1}^{n-1} \frac{r^{m}}{(m+2) !} \sum_{j=2}^{m+1}\left(\begin{array}{c}
m+2 \\
j
\end{array}\right) \beta_{j}(\lambda) \alpha_{m-j+3}(\lambda)+r^{n} y_{4}(r) \\
& =J_{2}+r^{n} y_{4}(r),
\end{aligned}
$$

we have

$$
\begin{aligned}
J_{2} & =\left(\sum_{m=2}^{n+1} \frac{\beta_{m}(\lambda)}{m !} r^{m-1}\right)\left(\sum_{m=1}^{n-1} \frac{\alpha_{m+1}(\lambda)}{m !} r^{m-1}\right)-r^{n} y_{4}(r) \\
& =(U(r)-w(r))\left(\sum_{m=1}^{n-1} \frac{\alpha_{m+1}(\lambda)}{m !} r^{m-1}\right)-r^{n} y_{4}(r) .
\end{aligned}
$$

Using (5.21), one may easily verify that

$$
\frac{\alpha_{m+1}(\lambda)}{m !}=\mu_{m-1}(\lambda)+(\sigma(\lambda)+1) \cdot \frac{\alpha_{m+1}(\lambda)}{(m+1) !}
$$

$(m=1,2, \cdots, n-1)$, so that

$$
\sum_{m=1}^{n-1} \frac{\alpha_{m+1}(\lambda)}{m !} r^{m-1}=\sum_{m=0}^{n-1} \mu_{m}(\lambda) r^{m}+(\sigma(\lambda)+1) \cdot \frac{1}{r}(P(r)-v(r))-\mu_{n-1}(\lambda) r^{n-1} .
$$

Hence

$$
\begin{aligned}
J_{2}= & (U(r)-w(r))\left\{\sum_{m=0}^{n-1} \mu_{m}(\lambda) r^{m}+(\sigma(\lambda)+1) \cdot \frac{1}{r}(P(r)-v(r))\right\} \\
& -\mu_{n-1}(\lambda) r^{n-1}(U(r)-w(r))-r^{n} y_{4}(r) .
\end{aligned}
$$


Substituting this into (12.5), we obtain

$$
\begin{aligned}
I_{11}= & \beta(\lambda) \sum_{m=0}^{n-1} \mu_{m}(\lambda) r^{m}+(U(r)-w(r)) \cdot \sum_{m=0}^{n-1} \mu_{m}(\lambda) r^{m} \\
& +(\sigma(\lambda)+1) \cdot(U(r)-w(r)) \cdot(1 / r)(P(r)-v(r)) \\
& -\mu_{n-1}(\lambda) r^{n-1}(U(r)-w(r)) \\
& (\sigma(\lambda)+1) \beta(\lambda) \cdot(1 / r)(P(r)-v(r))+r^{n} y_{3}(r)-r^{n} y_{4}(r) \\
= & (U(r)+\beta(\lambda)) \cdot \sum_{m=0}^{n-1} \mu_{m}(\lambda) r^{m}+(\sigma(\lambda)+1) \cdot(1 / r)(U(r)+\beta(\lambda)) P(r) \\
& +r^{n} y(r)+z_{1}(r) w(r)-(\sigma(\lambda)+1) \beta \cdot(1 / r) v(r),
\end{aligned}
$$

where

$$
\begin{gathered}
y(r)=y_{3}(r)-y_{4}(r)-\mu_{n-1}(\lambda) r^{n-1} \cdot \sum_{m=3}^{n+1} \frac{\beta_{m}(\lambda)}{m !} r^{m-1} \\
z_{1}(r)=-\sum_{m=0}^{n-1} \mu_{m}(\lambda) r^{m}-(\sigma(\lambda)+1) \cdot \sum_{m=2}^{n} \frac{\alpha_{m}(\lambda)}{m !} r^{m-2} .
\end{gathered}
$$

Recalling (12.4) and noticing that

$$
\left.\frac{d}{d r}\right|_{r=0}\left(K_{M}\left(c_{\lambda}(r)\right)-K_{N}\left(c_{\lambda}(r)\right)-2 K_{M}\left(c_{\lambda}(r)\right) p_{n}(r)\right)=0,
$$

we easily find that

$$
I_{12}-(\sigma(\lambda)+1) \beta(\lambda) \cdot \frac{1}{r} v(r)=r z_{2}(r) v(r)-K_{M}\left(c_{\lambda}(r)\right) v^{2}(r) .
$$

Hence

$$
\begin{aligned}
(U(r)+ & \beta(\lambda)) f_{\lambda}(r, P(r), U(r))=I_{11}+I_{12}-I_{2} \\
= & (U(r)+\beta(\lambda)) \cdot \sum_{m=0}^{n-1} \mu_{m}(\lambda) r^{m}+r^{n} y(r) \\
& +z_{1}(r) w(r)+r z_{2}(r) v(r)-K_{M}\left(c_{\lambda}(r)\right) v^{2}(r),
\end{aligned}
$$

and the lemma follows.

Formulas (6.18) and (6.19) follow immediately from Lemma 11.1. To prove (5.30), recall that $\alpha_{1}(\lambda)=\beta_{2}(\lambda)=0$, so that, by (5.28) and (5.29), (12.1) holds with $v(r)$ and $w(r)$ satisfying

$$
|v(r)| \leq C r^{n}, \quad|w(r)| \leq C r^{n+1} \quad \text { for } 0<r<\delta .
$$

Hence the right-hand side of (12.2) is bounded by $\mathrm{Cr}^{n}$. Since also

$$
|\beta(\lambda)+U(r)| \geq \frac{1}{2}|\beta(\lambda)|>0 \quad \text { for } 0<r<\delta,
$$

we see that (5.30) follows. 


\section{ACKNOWLEDGEMENT}

The first author is supported by the China National Natural Science Foundation (Grant 10171112) and the Distinguished Visiting Scholar Program of the China Scholarship Council. The second author is supported by the National Natural Science Foundation of USA Grant DMS-0098520. The first author wishes to thank the staff of the Department of Mathematics of The Ohio State University for their friendly hospitality when he was a visiting scholar from October, 2001 to September, 2002 .

\section{REFERENCES}

[1] J. Adam, A simplified mathematical model of tumor growth, Math. Biosci. 81(1986), 224229.

[2] N. Britton and M. Chaplain, A qualitative analysis of some models of tissue growth, Math. Biosci. 113(1993), 77-89.

[3] H. Byrne and M. Chaplain, Growth of necrotic tumors in the presence and absence of inhibitors, Math. Biosci. 135(1996), 187-216.

[4] S. Cui and A. Friedman, Analysis of a mathematical model of the effect of inhibitors on the growth of tumors, Math. Biosci. 164(2000), 103-137. MR 2001d:92006

[5] S. Cui and A. Friedman, Analysis of a mathematical model of the growth of necrotic tumors, J. Math. Anal. Appl. 255(2001), 636-677. MR 2002a:35195

[6] M. Dorie, R. Kallman and M. Coyne, Effect of cytochalasin b, nocodazole and irradiation on migration and internalization of cells and microspheres in tumor cell spheroids, Exp. Cell Res. 166(1986), 370-378.

[7] M. Dorie, R. Kallman, D. Rapacchietta and et al, Migration and internalization of cells and polystyrene microspheres in tumor cell spheroids, Exp. Cell Res. 141(1982), 201-209.

[8] A. Friedman and F. Reitich, Analysis of a mathematical model for the growth of tumors, $J$. Math. Biol. 38(1999), 262-284. MR 2001f:92011

[9] H. Greenspan, Models for the growth of solid tumor by diffusion, Stud. Appl. Math. 51(1972), 317-340.

[10] H. Greenspan, On the growth and stability of cell cultures and solid tumors, J. Theor. Biol. 56(1976), 229-242. MR 55:2183

[11] F. Hughes and C. McCulloch, Quantification of chemotactic response of quiescent and proliferating fibroblasts in Boyden chambers by computer-assisted image analysis, J. Histochem. Cytochem. 39(1991), 243-246.

[12] D. McElwain and G. Pettet, Cell migration in multicell spheroids: swimming against the tide, Bull. Math. Biol. 55(1993), 655-674.

[13] G. Pettet, C. P. Please, M. J. Tindall and et al, The migration of cells in multicell tumor spheroids, Bull. Math. Biol. 63(2001), 231-257.

[14] J. Sherrat and M. Chaplain, A new mathematical model for avascular tumor growth, J. Math. Biol. 43(2001), 291-312.

[15] K. Thompson and H. Byrne, Modelling the internalisation of labelled cells in tumor spheroids, Bull. Math. Biol. 61(1999), 601-623.

[16] J. Ward and J. King, Mathematical modelling of avascular-tumor growth II: Modelling growth saturation, IMA J. Math. Appl. Med. Biol. 15(1998), 1-42.

Institute of Mathematics, Zhongshan University, Guangzhou, Guangdong 510275, People's Republic of China

E-mail address: mcinst@zsu.edu.cn

Department of Mathematics, The Ohio State University, 231 West 18th Avenue, Columbus, OHiо 43210-1174

E-mail address: afriedman@mbi.osu.edu 